University of Rhode Island

DigitalCommons@URI

Open Access Dissertations

2017

\title{
Sub-Terahertz Range Fiber Optic Devices for Sensing Applications
}

Zhen Chen

University of Rhode Island, eechen@uri.edu

Follow this and additional works at: https://digitalcommons.uri.edu/oa_diss

\section{Recommended Citation}

Chen, Zhen, "Sub-Terahertz Range Fiber Optic Devices for Sensing Applications" (2017). Open Access Dissertations. Paper 629.

https://digitalcommons.uri.edu/oa_diss/629

This Dissertation is brought to you for free and open access by DigitalCommons@URI. It has been accepted for inclusion in Open Access Dissertations by an authorized administrator of DigitalCommons@URI. For more information, please contact digitalcommons-group@uri.edu. 
SUB-TERAHERTZ RANGE FIBER OPTIC DEVICES FOR

SENSING APPLICATIONS

BY

ZHEN CHEN

A DISSERTATION SUBMITTED IN PARTIAL FULFILLMENT OF THE REQUIREMENTS FOR THE DEGREE OF

DOCTOR OF PHILOSOPHY

IN

ELECTRICAL ENGINEERING

UNIVERSITY OF RHODE ISLAND 


\section{DOCTOR OF PHILOSOPHY DISSERTATION}

$\mathrm{OF}$

ZHEN CHEN

APPROVED:

Dissertation Committee:

$\begin{array}{ll}\text { Major Professor } & \text { Tao Wei } \\ & \text { Yan Sun } \\ & \text { Otto J. Gregory } \\ & \text { Nasser H. Zawia } \\ & \text { DEAN OF THE GRADUATE SCHOOL }\end{array}$

UNIVERSITY OF RHODE ISLAND 


\begin{abstract}
Distributed sensing refers to the solution which enables the real-time, continuous measurement at multiple sensing locations (typically, more than 100 sensing nodes). Due to many of its unique advantages, such as small size, light weight, low cost, electromagnetic immunity, high-temperature survivability, and chemical stability, optical fibers have been well accepted as one of the most promising candidates as the platform for distributed sensing applications.

Among different fiber distributed sensing methods, optical frequency domain reflectometry (OFDR) represents a particular promising candidate. Based on the frequency modulated continuous wave (FMCW) method, OFDR method is capable of measuring the spatial-continuous weak Rayleigh scattering patterns along the entire length of the fiber under test, with high spatial resolution $(\sim \mu \mathrm{m})$ level and moderate interrogation distance $(\sim \mathrm{km})$. To extract the structural information from the unmodified communication grade single mode fiber, large interrogation bandwidth is needed. However, this resource of optical bandwidth is very expensive. The external cavity laser is the state-of-the-art frequency sweep laser source for OFDR system, which costs at least \$20,000. The cost has rendered the OFDR interrogation technique expensive and limits its applications.

This dissertation focused on the improvement of OFDR interrogation system by reducing the total system cost. First, a series of sub-terahertz range fiber sensors, including Fabry-Perot cavity sensors and terahertz fiber grating sensors were symmetrically investigated. Fabricated using femtosecond laser micromachining techniques, these sensors allow the OFDR system for low bandwidth interrogation while
\end{abstract}


maintaining the high accuracy measurement. In addition, a sensor fabrication method without stripping the fiber polymer buffer was developed. This is the first time that an in-line grating structure has been fabricated within the core of an optical fiber with an intact buffer coating, allowing the fiber to retain optimal mechanical properties.

Second, a series of low cost sweep laser sources were developed as high-linear coherent sweep laser source, suitable for the sub-terahertz range fiber sensor interrogation. Based on current injection modulation methods, the semiconductor lasers were used as the sweep laser sources and the output wavelength was feedback controlled using optical phase locked loop techniques. The method of using VCSEL laser was also investigated to increase the sweep bandwidth. In addition, an all-digital optical phase locked loop system was implemented using the field programmable gate array, which increases the system design flexibility. 


\section{ACKNOWLEDGMENTS}

Frist and foremost, I would like to express my most sincere gratitude to my major professor, Prof. Tao Wei for his patience, kindness, and guidance throughout my Ph.D. study at URI. It is my great horror of being accepted as his very first student, having the opportunity to work on a broad range of interesting fiber sensing research topics. Thank you for being supportive even when I feel like I can't do it, and always pushing me to do my best.

I am very grateful to have Prof. Yan Sun, Prof. Otto Gregory and Prof. Yi Zheng for being my dissertation committee members and their support in correcting my dissertation. I also want thank Prof. Haibo He, Prof. Qing Yang, Prof. Steven Kay, and Prof. Jimmy Oxley for all their valuable advice for my course work and research projects URI.

I would like to thank my research collaborators and friends at URI, including Yihai Zhu, Yongbo Zeng, Jun Yan, Bo Tang, Yufei Tang, Xiangnan Zhong, Shuyi Pei, Dongyang Li, Zhenghan Zhu, Xin Zhou, Yazan Rawashdeh, Ryan Rettinger and Zheyi Yao. Many thanks should go to Yuan Lei at Clemson University for all the great work. Thanks to my roommate Bradley Scott for all the best time we had.

Special thanks to my colleague and friend Gerry Hefferman for your advice, enthusiasm and help. I am very fortunate to have you team-up together during my Ph.D. research work.

Last, I would like to thank my parents for their unconditional trust and love. There are no words to express my gratitude to you. Thanks to my girlfriend Yining Li who is being my source of joy and happiness. 


\section{PREFACE}

This dissertation is organized in the manuscript format consisting of eight manuscripts as following:

- Manuscript 1:

Z. Chen, L. Yuan, G. Hefferman and T. Wei, "Ultra-weak intrinsic FP cavity array for distributed sensing", published in Optics Letters, vol.40, no.3, pp.320-323, Feb. 2015.

- Manuscript 2:

Z. Chen, L. Yuan, G. Hefferman and T. Wei, “Terahertz fiber Bragg grating for distributed sensing", published in Photonics Technology Letters, IEEE, vol. 27, no. 10, pp. 1084 - 1087, May. 2015.

- Manuscript 3:

Z. Chen, G. Hefferman, L. Yuan, Y. Song and T. Wei, "Ultra-weak waveguide modification with femtosecond laser pulses", published in Photonics Technology Letters, IEEE, vol. 27, no. 16, pp. 1705 - 1708, Jun. 2015.

- Manuscript 4:

G. Hefferman, Z. Chen and T. Wei, "Phase-shifted terahertz fiber Bragg grating for strain sensing with large dynamic range", published in Photonics Technology Letters, IEEE, vol. 27, no. 15, pp. 1649 - 1652, Jun. 2015.

- Manuscript 5:

Z. Chen, G. Hefferman, L. Yuan, Y. Song and T. Wei, “Terahertz-range interrogated grating-based two-axis optical fiber inclinometer", published in Optical Engineering, SPIE, vol. 55, no. 2, pp. 026106, Feb. 2016. 
- Manuscript 6:

Z. Chen, G. Hefferman and T. Wei, “A low bandwidth DFB laser-based interrogator for terahertz-range fiber Bragg grating sensors", published in Photonics Technology Letters, IEEE, vol. 29, no. 4, pp. 365 - 368, Feb. 2017.

- $\quad$ Manuscript 7:

Z. Chen, G. Hefferman and T. Wei, "Digitally controlled chirped pulse laser for subterahertz-range fiber structure interrogation”, published in Optics Letters, vol. 42, no. 5, pp. 1007-1010, Mar. 2017

- Manuscript 8:

Z. Chen, G. Hefferman and T. Wei, "Field-programmable gate array-controlled sweep velocity-locked laser pulse generator", published in Optical Engineering, SPIE, vol. 56, no. 5, pp. 054102, May. 2017. 


\section{TABLE OF CONTENTS}

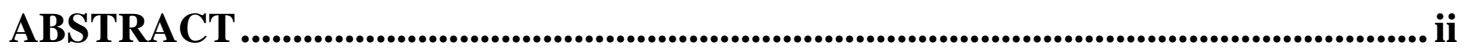

ACKNOWLEDGMENTS ....................................................................................................... iv

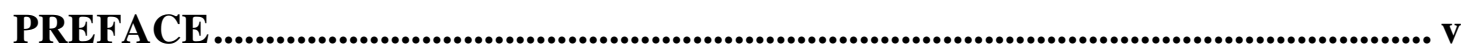

TABLE OF CONTENTS...................................................................................... vii

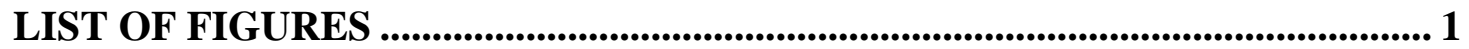

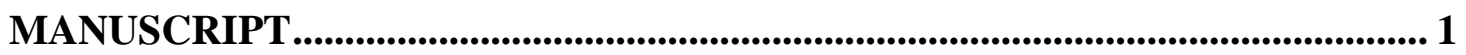

1. Ultraweak intrinsic Fabry-Perot cavity array for distributed sensing ............ 1

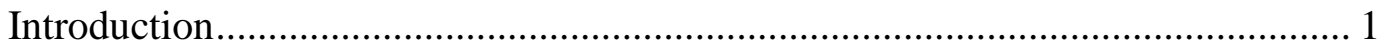

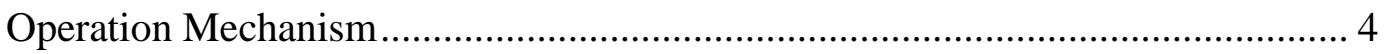

Experimental Results and Discussions ……………….................................. 8

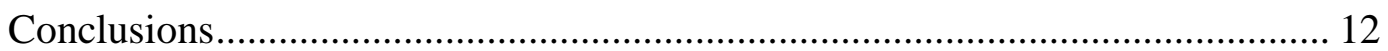

2. Terahertz fiber Bragg grating for distributed sensing ....................................... 14

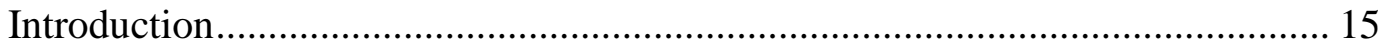

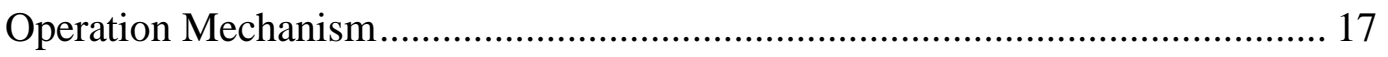

Experimental Results and Discussions ........................................................... 20

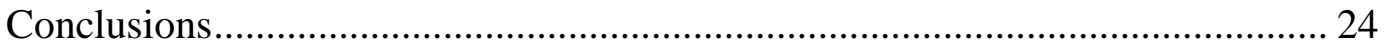

3. Ultra-weak waveguide modification with femtosecond laser pulses............... 25

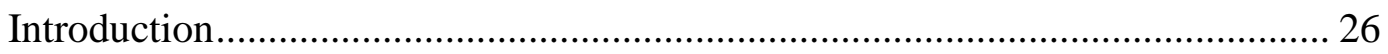

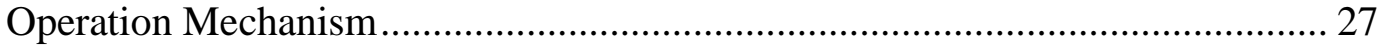

Experimental Results and Discussions ............................................................. 29

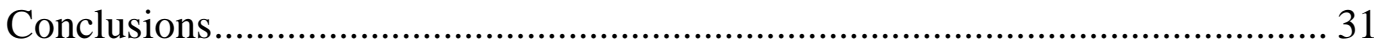

4. Phase-shifted terahertz fiber Bragg grating for strain sensing with large dynamic range........................................................................................................................ 32

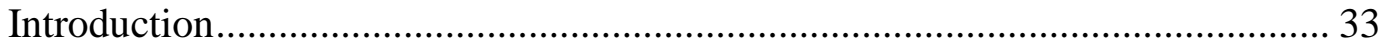

Operation Mechanism.................................................................................... 35

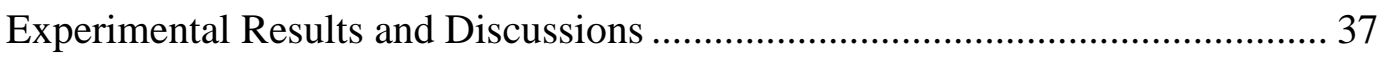

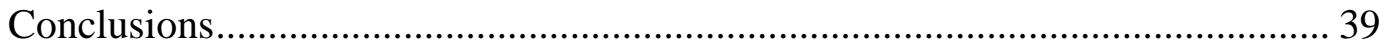


5. Terahertz-range interrogated grating-based two-axis optical fiber

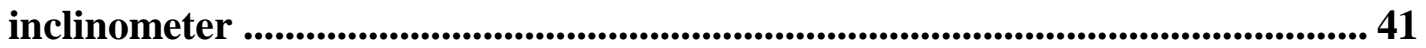

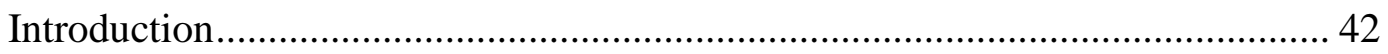

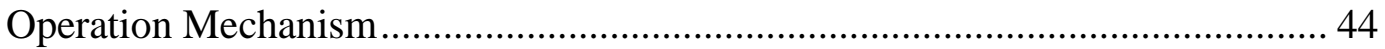

Experimental Results and Discussions ....................................................... 49

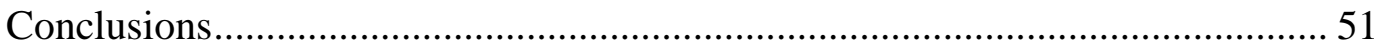

6. A low bandwidth DFB laser-based interrogator for terahertz-range fiber

Bragg grating sensors........................................................................................................ 52

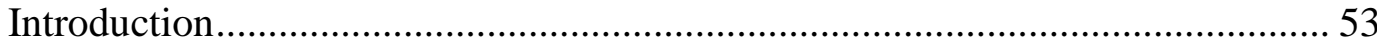

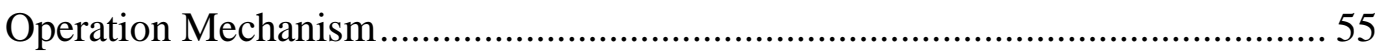

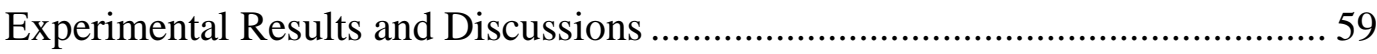

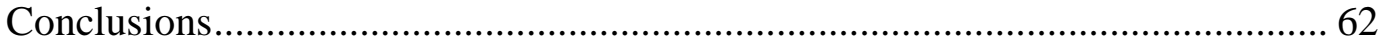

7. Digitally controlled chirped pulse laser for sub-terahertz-range fiber structure interrogation.......................................................................................... 63

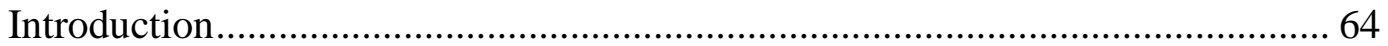

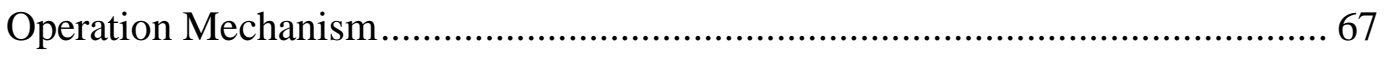

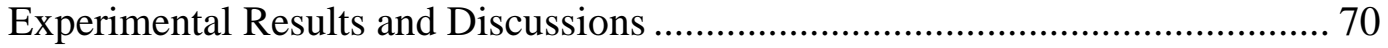

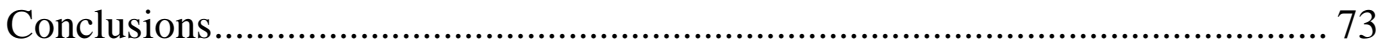

8. Field-programmable gate array-controlled sweep velocity-locked laser pulse

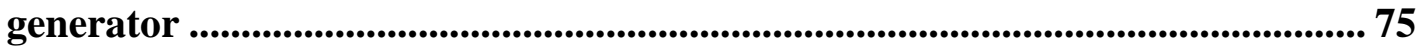

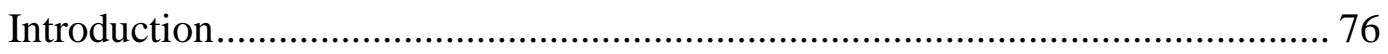

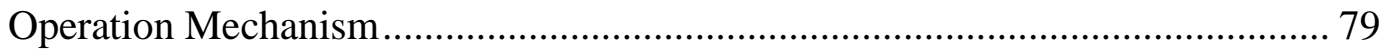

Experimental Results and Discussions ....................................................... 83

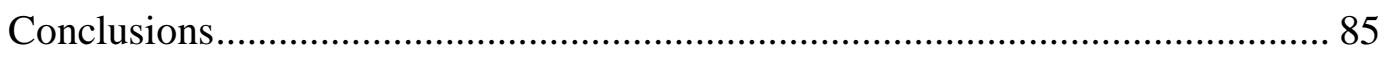

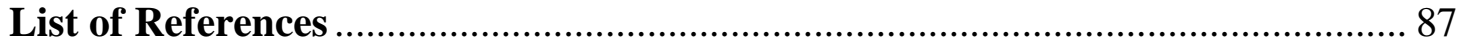




\section{LIST OF FIGURES}

FIGURE

PAGE

Figure 1.1 Schematic of the interrogation system for IFPI arrays 4

Figure 1.2 Microscopic image and reflection distribution in spatial domain of three 1 cm IFPI cavities fabricated using differing laser power: (a) 0.14 , (b) 0.12 , and (c) 0.1

W. 5

Figure 1.3 Temperature responses of IFPIs of differing lengths: (a) interferogram of a 1 cm IFPI at $63^{\circ} \mathrm{C}$ and $65^{\circ} \mathrm{C}$; (b) interferogram of a $1 \mathrm{~mm}$ IFPI at $63^{\circ} \mathrm{C}$ and $65^{\circ} \mathrm{C}$; and (c) frequency shifts as a function of temperature for both $1 \mathrm{~cm}$ and $1 \mathrm{~mm}$ IFPIs............. 8 Figure 1.4. Demonstration of ultra-weak IFPIs for distributed sensing, where only the $2^{\text {nd }}$ IFPI is subject to temperature change: (a) reflection distributions of 3 IFPIs; (b) interferogram of $1^{\text {st }}$ IFPI when ambient temperature of $2^{\text {nd }}$ IFPI is varied; (c) interferogram of $2^{\text {st }}$ IFPI at $63^{\circ} \mathrm{C}$ and $65^{\circ} \mathrm{C}$; (d) interferogram of $3^{\text {rd }}$ IFPI when temperature of $2^{\text {nd }}$ IFPI is varied. 9

Figure 1.5 Temperature response of a $1 \mathrm{~cm}$ IFPI interrogated using different laser sweeping ranges: (a)-(c) interferogram of the IFPI (d) temperature response with 3 interrogation bandwidths. 10

Figure 1.6 Experimental study of continuously cascaded ultra-weak IFPIs: (a) test setup; (b) reflection distribution of 5 IFPIs; (c) frequency distribution measured using IFPI array...... 11

Figure 2.1 Schematic of the interrogation system for THz FBG 17

Figure 2.2 Fabrication of THz FBG with femtosecond laser 18 
Figure 2.3 Simulation and experimental results: (a) $1 \mathrm{~mm}, 20$ reflection points, $0.11 \mathrm{~W}$; (b) $0.1 \mathrm{~mm}, 20$ reflection points, $0.11 \mathrm{~W}$ 19

Figure. 2.4. Temperature response: (a) $1 \mathrm{~mm} \mathrm{THz} \mathrm{FBG} \mathrm{reflection} \mathrm{spectra} \mathrm{at} 65^{\circ} \mathrm{C}$ and $55^{\circ} \mathrm{C}$; (b) cross-correlation pattern to extract the frequency shift from (a); (c) frequency shift as a function of temperature 21

Figure 2.5. Distributed sensing test with THz FBG array: (a); a time-domain 40 reflection point THz FBG signal; (b) frequency-shift distribution measured using THz FBG array. 22

Figure 2.6. Temperature response of $1 \mathrm{~mm} \mathrm{THz}$ FBG using different laser sweeping ranges: (a) - (c) temperature response with 3 interrogation bandwidth, (d) temperature

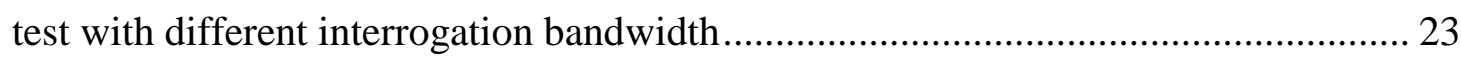

Figure 3.1 Schematic of femtosecond laser fabrication ............................................ 28 Figure 3.2 Distance domain signals for THz FBG fabricated by femtosecond laser: (a) with buffer coating stripped; (b) proposed method with buffer 29 Figure 3.3 Strain and temperature test with two different THz FBGs: (a) strain test results; (b) temperature test results 30

Figure 4.1 Experimental set-up and time-domain plots of the optical fiber under test. (a) Diagram of the strain testing set-up; (b) time domain plot of optical fiber with both the $\pi$-phase-shifted and unmodified THz FBGs in series; (c) detail of the time-domain trace at the area of the $\pi$-phase-shifted THz FBG; (d) detail of the time-domain trace at the area of the unmodified THz FBG 34 
Figure 4.2 Simulation results and experimentally collected spectrum of the $\pi$-phaseshifted THz FBG 36

Figure 4.3 Experimental results of strain testing along the optical fiber under test. (a) Spectra of $\pi$-phase-shifted THz FBG; (b) spectra of unmodified THz FBG; (c) relative peak shift of the $\pi$-phase-shifted THz FBG as a function of strain and best-fit trend line; (d) relative peak shift of the unmodified THz FBG as a function of strain and best-fit trend line. Points and curves of the same color were collected and calculated simultaneously as weights were sequentially added to the fiber under test. 38

Figure 5.1 Three-core fiber inclinometer bundle packaging and assembling: (a) before heated, (b) after heated, (c) schematic of the assembling the fiber inclinometer. 45 Figure 5.2 Schematic of cascaded THz FBG sensors: (a) the time domain interrogation signal of three identical THz FBG sensing arrays, (b) the details of sensing array 1, (c) the packaged inclinometer bundle. 46

Figure 5.3 Schematic of the measured inclination angle: (a) 3D representation, (b) 2D representation (project to $\mathrm{YZ}$ plane) 47

Figure 5.4 Calibrate the relative position: (a) - (c): the cross-plane illustration of bending with sensing array \#1, \#2 and \#3 on top respectively, (d) schematic of inclination bending...... 48

Figure 5.5 Calibrate the relative position: (a) with sensing array \#1 on top, the strain measurement result along the three sensing arrays, (b) the solved relative position on the $1^{\text {st }}$ sensing element cross-plane 
Figure 5.6 Two-axis inclination measurement results: (a) measured inclination angles $\theta$ $=0^{\circ}, 0.767^{\circ}$ and $1.70^{\circ}$, respectively, at eight differing azimuthal angles $(\varphi)\left(45^{\circ}\right.$ apart from $0^{\circ}$ to $315^{\circ}$ ) (b) measured inclination angle against calculated angle at $\varphi=0^{\circ}$, (c) measured inclination angle against calculated angle at $\varphi=270^{\circ}$ 50

Figure 6.1 Schematic of the chirp-DFB laser-based C-OFDR interrogation system (LDC: laser diode controller; AWG: arbitrary wave generator; DAQ: data acquisition; DFB: distributed feed-back laser; CPL: coupler; CIR: circulator; DUT: devices under test; BPD: balanced photodiode) 55

Figure 6.2 The measured MZI radio frequency, or the chirp-DFB laser sweeping speed as a function of time using different voltage modulation waveforms: (a) an initial ramp input; (b) after 6 feedback pre-distortion iterations. 56 Figure 6.3 Strain tests using the chirp-DFB laser source: (a) strain sensing test setup; (b) time domain reflection signals of the DUT with a $0.12 \mathrm{~W}$ THz FBG; (c) frequency domain interferograms with varying applied strain values; (d) resulting frequency shift at the varying applied strain values. 58

Figure 6.4 Stability test results using 200 iterative measurements 58 Figure 6.5 Multiplexing test using two THz FBG sensors: (a) multiplexing test setup; (b) time domain reflection signals of the DUT with both the $0.12 \mathrm{~W} \mathrm{THz} \mathrm{FBG} \mathrm{and} \mathrm{the}$ $0.14 \mathrm{~W}$ THz FBG; (c) frequency shift interferograms of the $0.12 \mathrm{~W}$ sensor with different strain values applied; (d) frequency interferograms of the $0.14 \mathrm{~W}$ sensor with no additional strain applied during the same period. 60 
Figure 7.1 Schematic of the proposed Sub-THz-FS interrogation system using SVLLPG. (ADC: analog-to-digital converter, DSP: digital signal processing unit, MZI: Mach-Zehnder Interferometer, AGC: automatic gain control amplifier.) 67

Figure 7.2 Spectrogram of AGC output within a chirped laser pulse: (a) free running; (b) sweep velocity locked; (c) modulation waveforms when the laser sweep velocity is locked. 68

Figure 7.3 Quality of sweep velocity locking: (a) Fourier transform of the AGC output over the locked span of $8.3 \mathrm{~ms}$; (b) a Gaussian curve fit applied to the measure the FWHM of the Fourier transform of a chirped pulse train over 1 second. 70 Figure 7.4 Static strain test: (a) time domain reflections of DUT; (b) interferograms of the sensor unit between $3975 \mathrm{~mm}$ and $3979 \mathrm{~mm}$ with varied strain applied; (c) strain test results for all 9 sensor units; (d) strain test results for 8th sensor unit 71 Figure 7.5 Distributed temperature test: (a) schematic; (b), (c) testing results [Visualization 1]. 72

Figure 8.1 Schematic of proposed all-digital OPLL system with sensing modulate and control module (MZI: Mach-Zehnder interferometer; FPGA: field-programmable logic array; DAC: digital-to-analog converter; ADD: adder; PRE: pre-distortion curve; LC: loop controller; REF: reference frequency clock; PD: type-II phase detector; ADC: analog-to-digital converter; DSP: digital signal processing unit). 79

Figure 8.2 Measured frequency spectrum of AGC output within a chirped laser pulse: (a) free running with ramp input; (b) free running with calibrated pre-distortion input; (c) sweep velocity locked. 81 
Figure 8.3 (a) Fourier transform of the AGC output over the locked span of 9 ms; (b) output of the laser pulse generator with 5 complete cycles; (c) a Gaussian curve fit applied to measure the FWHM of the Fourier transform of a chirped pulse train over 1

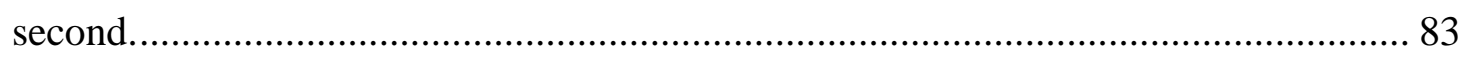

Figure 8.4 Static strain test: (a) time domain reflections of DUT; (b) interferograms of the sensor unit between $1779 \mathrm{~mm}$ and $1783 \mathrm{~mm}$ with varied strain applied; (c) strain test results for all 9 sensor units; (d) strain test results of for 7th sensor unit 84 


\title{
MANUSCRIPT
}

1. Ultraweak intrinsic Fabry-Perot cavity array for distributed sensing by

Zhen Chen, Lei Yuan, Gerald Hefferman and Tao Wei

\author{
published in
}

Optics Letters, vol. 30, no. 3, pp.320-323, Feb. 2015. 


\begin{abstract}
This Letter reports on an ultraweak intrinsic Fabry-Perot interferometer (IFPI) array fabricated by a femtosecond (fs) laser for distributed sensing applications. Ultralow reflectors $(<-60 \mathrm{~dB})$ were obtained. IFPIs with different physical lengths showed identical temperature sensitivity $(-1.5 \mathrm{GHz} / \mathrm{C})$. A distributed temperature sensing test was conducted. No crosstalk between IFPI elements in the array was observed, implying the device's utility as a distributed sensing system. The possibility of using smaller bandwidths for sensor interrogation was experimentally proven. A small-scale temperature distribution test was conducted on a continuously cascaded ultraweak IFPI array, demonstrating its high spatial resolution. The temperature detection limit of this system was measured to be less than $0.0667^{\circ} \mathrm{C}$.
\end{abstract}

\title{
Introduction
}

Distributed optical fiber sensing technology is a thriving branch of sensing technology, due in large part to its ability to surmount many limitations of traditional single-point sensor, enabling a single system to simultaneously span a large number of equivalently individual sensors [1]. These unique advantages have been successfully demonstrated in many application areas, including oil drilling, structural health monitoring, and perimeter security [2]. The recent development of distributed optical fiber sensors with high spatial resolution has expanded this utility considerably, making it an attractive addition to many emerging applications, such as wearable devices, robotics, and surgical instrument [3, 4].

The use of Rayleigh scattering as a sensing method has shown particular promise in distributed sensing with high spatial resolution [5]. The unique Rayleigh 
backscattering profile of a section of optical fiber can be extracted via swept-frequency interferometry, revealing the profile of ambient temperature and strain change along the length of the fiber. By sweeping over a broad optical bandwidth, this technology can resolve minute changes in Rayleigh backscattering profile with mm-level spatial resolution. However, this modality requires the interrogating laser to sweep over a broad wavelength range with high coherence length, limiting the update rate of the measurement system. Additionally, broadband, highly coherent, and single longitudinal mode swept lasers come at considerable cost.

Weak fiber Bragg grating (FBG) arrays represent another technology breakthrough in this area [6]. A large number of low reflection FBGs with same nominal resonant wavelength are fabricated along an optical fiber in a discrete fashion, known as weak FBG sensor network, or in a continuous fashion, known as long-length FBG [7]. The sensing mechanism of an individual weak FBG is the same conventional FBG; however, in contrast to the wavelength domain multiplexing (WDM) technology used for traditional FBGs, a weak FBG array is multiplexed in the spatial domain. The low reflectivity of each individual FBG element ensures minimal crosstalk among FBGs in an array. Researchers have demonstrated several probing technologies to interrogate weak FBG arrays, including combined wavelength-time-domain reflectometry, optical frequency domain reflectometry (OFDR), and synthesis of optical coherence function (SOCF) method [7]. The responses of thousands of individual weak FBGs in a single array can be measured simultaneously. In comparison with the Rayleigh scattering approach, weak FBGs require a significantly smaller wavelength range for sensor 
interrogation, leading to a higher update rate. Currently, a weak FBG array is fabricated during the fiber drawing process and features a reflectivity of $\sim-33 \mathrm{~dB}[8]$.

There exists another highly sensitive, miniature-scale, and low reflection device in the optical fiber sensor family - intrinsic Fabry-Pérot interferometer (IFPI). This technology uses a pair of small reflectors introduced in an optical fiber, forming a cavity that creates interferogram in the wavelength domain. Ambient physical changes are detected through shifts of the resulting interferogram. Traditionally, the reflectors of IFPIs are fabricated via splicing together different optical fibers of differing refractive indices or via exposing a fiber to intense UV light [9]. Recent technology advances have led to increased popularity in the use of femtosecond (fs) lasers in fabricating fiber optic devices $[10,11]$. Weak reflectors with a reflectivity of $\sim-45 \mathrm{~dB}$ were achieved to form IFPIs with this fabrication method [12]. An FFT-based method was used to multiplex 3 IFPIs of differing cavity lengths. Very recently, Huang et al. successfully demonstrated an optical carrier based microwave interferometry method to simultaneously interrogate identical and cascaded IPFIs with a length of $12 \mathrm{~cm}$ along an optical fiber [13]. However, the low microwave frequency bandwidth limits its spatial resolution.

This letter reports a fiber-inline ultra-weak $(<-60 \mathrm{~dB})$ IFPI array fabricated using fs laser for distributed sensing with high spatial resolution. Interrogation approach, fabrication parameters, sensitivity, operation bandwidth, and distributed sensing ability of the proposed IFPI array were experimentally investigated in this letter. 


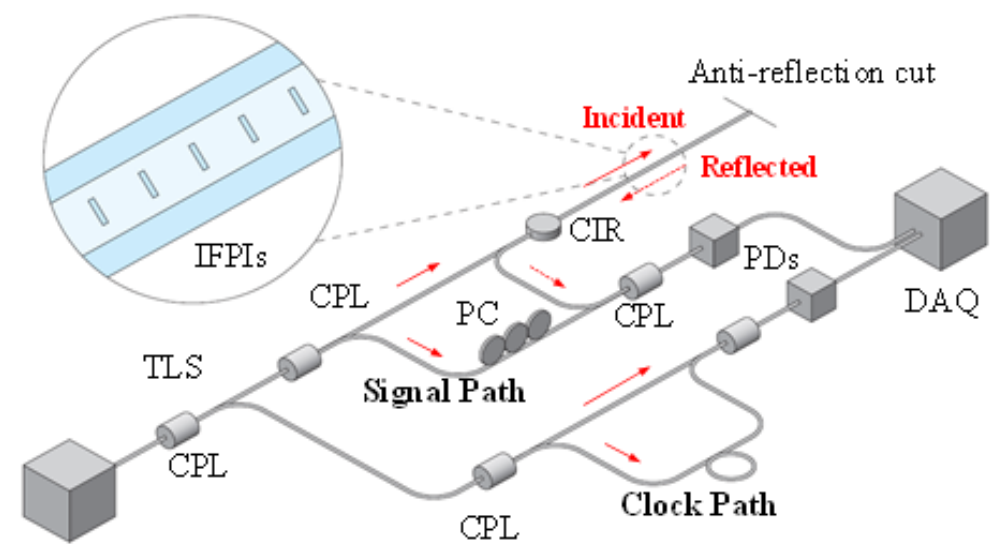

Figure 1.1 Schematic of the interrogation system for IFPI arrays

\section{Operation Mechanism}

The Schematic of the interrogation system, based on OFDR, is drawn in Figure 1.1 Light from a tunable laser source (TLS, Newport 6428) is split into two paths - "clock" and "signal". "Clock" is an interferometer used to calibrate the non-linear sweep effect of the TLS by providing a corrected time base for a data acquisition card (DAQ, NI 6251 with the sampling rate of $1 \mathrm{MHz}$ ) during frequency sweep. Light in the "signal" section is split between the reference and measurement arms of an interferometer via a 50/50 coupler (CPL); in the measurement path, an optical circulator (CIR) further splits the light to interrogate the low-reflection IFPI array and returns the reflected light. A polarization controller (PC) is used to tune the state of polarization in the system. Another 50/50 CPL then recombines the measurement and reference fields. In this setup, the TLS sweeps from 1535 to $1565 \mathrm{~nm}$ at a speed of $16 \mathrm{~nm} / \mathrm{s}$, covering a total bandwidth of $3.7 \mathrm{THz}$. Thus, the AC-coupled voltage received by the DAQ is written as:

$$
v_{\text {total }}=2 \eta r I_{\text {ref }} \sum_{n=1}^{N-1} \cos \left[\beta\left(Z_{\text {ref }}-Z_{n}\right)\right]
$$

where $\eta$ is the light-to-voltage conversion coefficient of the photodetector, $\mathrm{r}$ is the reflection coefficient of the reflector of the IFPI, $\mathrm{I}_{\text {ref }}$ is the light intensity of the reference 
arm, $\mathrm{N}$ is the number of reflectors along the optical fiber, $\beta$ is the propagation constant, $Z_{\text {ref }}$ is the length of reference fiber, and $z_{n}$ represents the position of $n$th reflector. The intensity of the reflected light as a function of position can be readily obtained via a Fourier transform. It is worth noting that Rayleigh backscatter in this system is considered as noise floor in this study. Two neighboring reflectors form an IFPI, which can be time-gated and extracted via a digital band-pass filter. The real-time system samples at 3 seconds per round. The data collected by DAQ is transmitted and processed on computer.
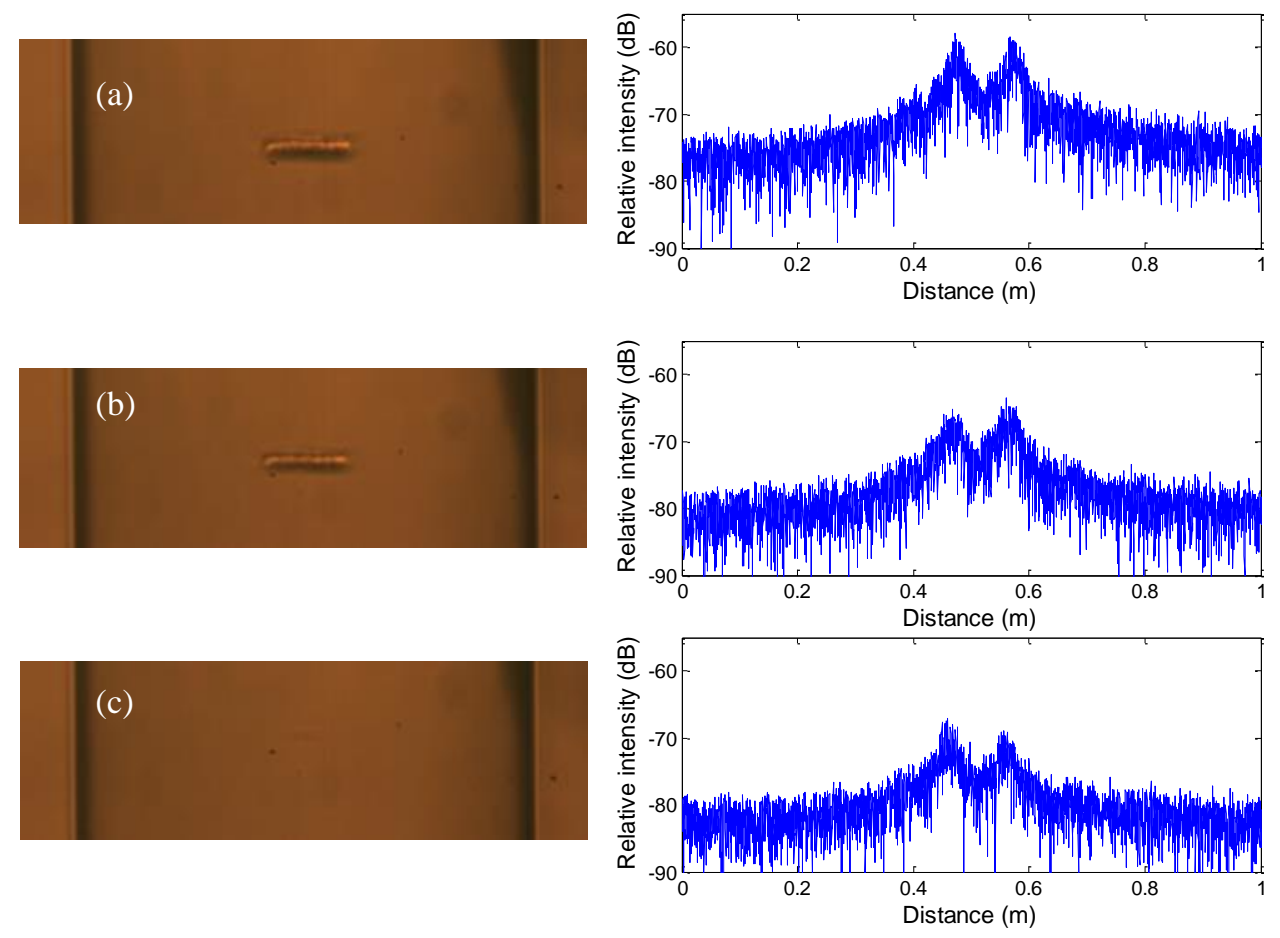

Figure 1.2 Microscopic image and reflection distribution in spatial domain of three $1 \mathrm{~cm} \mathrm{IFPI}$ cavities fabricated using differing laser power: (a) 0.14 , (b) 0.12 , and (c) $0.1 \mathrm{~W}$.

A Ti: Sapphire fs laser (Coherent, Inc.) micromachining system was used to fabricate ultra-weak reflectors. The central wavelength, pulse width, repetition rate, and maximum power of the laser are $800 \mathrm{~nm}, 200 \mathrm{fs}, 250 \mathrm{kHz}$, and $1 \mathrm{~W}$, respectively. The actual power used for fabrication is controlled by adjusting a half-wave plate, a 
polarizer, and several neutral density (ND) filters. The laser was switched on or off by electrically gating the internal clock. A single-mode optical fiber (Corning, SMF-28e) with the core and cladding diameters of 8.2 and $125 \mu \mathrm{m}$, respectively, was used for these experimental trials. After mechanically stripping its buffer, the fiber was cleaned using acetone and clamped onto two bare fiber holders, which were immersed in distilled water during fabrication. The fiber assembly was mounted on a computer-controlled three-axis translation stage with a resolution of $0.1 \mu \mathrm{m}$ (Newport, Inc.). The fs laser beam was focused inside the optical fiber through a water immersion objective lens (Olympus UMPlanFL 20x) with a numerical aperture (NA) of 0.4. The velocities of the stages were set at $50 \mu \mathrm{m} / \mathrm{s}$ during fabrication. A cuboid region $(10 \times 2 \times 10 \mu \mathrm{m})$ was inscribed in the center of the fiber from bottom up to cover the whole cross-section of the fiber core. The center of the inscribed region was aligned with the center of the fiber core. Figure 1.2 shows both the microscopic images and reflection distribution of three IFPIs fabricated with different fs laser powers $(0.14,0.12$, and $0.1 \mathrm{~W})$. The reflection values measured using the interrogation system were referenced to an angled polished connector (APC), which was previously measured using off-the-shelf precision instruments. The reflectivity of the weak reflectors decreased as fabrication power was reduced. A reflectivity of around $-70 \mathrm{~dB}$ was achieved with laser beam at $0.1 \mathrm{~W}$. In this study, $0.14 \mathrm{~W}$ was chosen as the fs laser power used for IFPI fabrication.

The sensing mechanism of an IFPI is based on tracking the phase shift of the interferogram in response to ambient change. To extract the phase shift information from the signal received from an individual IFPI, the reflection signal in frequency- 
domain is squared and filtered using a low-pass filter to obtain the interference signal $(\mathrm{S})$ :

$$
S=4 \eta^{2} r^{2} I_{r e f}^{2} \cos \left(4 \pi n_{e f f} L_{f} / c\right)
$$

where $\mathrm{L}$ is the physical cavity length of IFPI, $\mathrm{n}_{\mathrm{eff}}$ is the effective refractive index of the fiber, $\mathrm{f}$ is the frequency of the laser, and $\mathrm{c}$ is the velocity of light in vacuum. Additionally, $\mathrm{n}_{\text {effL }}$ is considered the optical length of the IFPI cavity. From Equation (2), as optical length increases, the period of the cosine function with respect to laser frequency decreases. As a result, at laser frequencies $(\sim 193 \mathrm{THz})$, the interference signal shifts to smaller frequencies, proportionally.

To investigate ultra-weak IFPI sensors, two IFPIs with lengths of $1 \mathrm{~cm}$ and $1 \mathrm{~mm}$, respectively, were fabricated. The temperature response of both IFPIs was measured using a temperature-controlled water bath. Figure 1.3 (a) and 1.3(b) plot the measured interferogram of both IFPIs at $63^{\circ} \mathrm{C}$ and $65^{\circ} \mathrm{C}$, where the $\mathrm{x}$-axis is the frequency offset from the start of frequency sweep. As temperature increases, the optical length of both IFPIs increases, leading to an interferogram shift towards lower frequency. The frequency shift of interferogram is determined via tracking its zero-crossing position. This technique is justified by the fact that steepest slope of a sinusoidal function occurs at its zero-crossing position. Figure 1.3(c) depicts the frequency shift of the interferogram as a function of temperature for both IFPIs. The temperature sensitivity of both $1 \mathrm{~cm}$ and $1 \mathrm{~mm}$ IFPIs was measured to be around $-1.5 \mathrm{GHz} /{ }^{\circ} \mathrm{C}$, agreeing well with the theory value [12]. Comparing the two IFPIs, the interference period with respect to frequency for the $1 \mathrm{~cm}$ IFPI is $1 / 10$ of the $1 \mathrm{~mm}$ IFPI. As a result, the $1 \mathrm{~cm}$ IFPI is more subject to $2 \pi$ ambiguity problem, limiting its measurement dynamic range. 
However, the slope of the $1 \mathrm{~cm}$ IFPI at zero-crossing position is ten times higher than that of the $1 \mathrm{~mm}$ IFPI, leading to a much higher accuracy. As a result, an IFPI with a longer length is suitable for precisely measuring smaller physical changes.
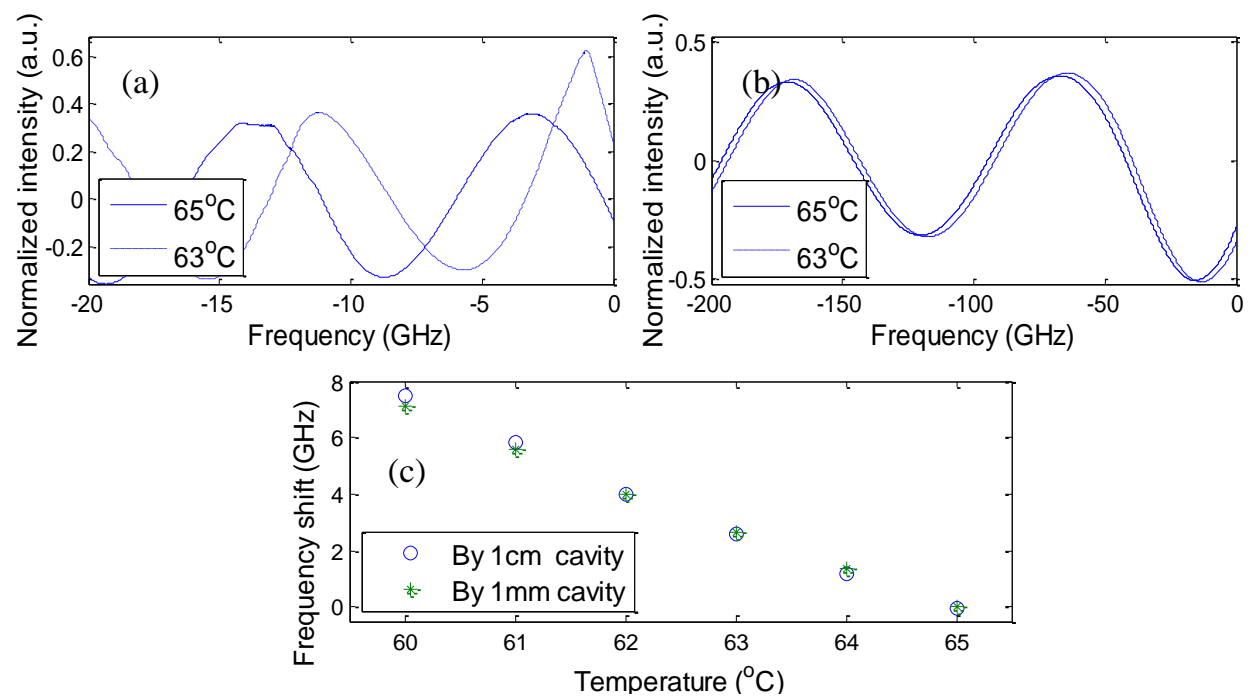

Figure 1.3 Temperature responses of IFPIs of differing lengths: (a) interferogram of a $1 \mathrm{~cm}$ IFPI at $63^{\circ} \mathrm{C}$ and $65^{\circ} \mathrm{C}$; (b) interferogram of a $1 \mathrm{~mm}$ IFPI at $63^{\circ} \mathrm{C}$ and $65^{\circ} \mathrm{C}$; and (c) frequency shifts as a function of temperature for both $1 \mathrm{~cm}$ and $1 \mathrm{~mm}$ IFPIs.

\section{Experimental Results and Discussions}

To demonstrate the distributed sensing capability of ultra-weak IFPIs, three identical $1 \mathrm{~cm}$ IFPIs were fabricated and spliced along a single fiber line. Figure 1.4 (a) depicts the reflection distributions of the 3 ultra-weak-IFPIs, which were spaced approximately $1.5 \mathrm{~m}$ apart. The $2 \mathrm{nd}$ IFPI (middle) was immersed in a temperaturecontrolled water bath, while the 1st and 3rd IFPIs were left unchanged at ambient temperature. The water bath was heated to $65 \mathrm{oC}$ and cooled down to $63 \mathrm{oC}$. Three bandpass filters were used to gate the 3 IFPIs in time-domain and extract the interferograms of all 3 IFPIs in frequency-domain, shown in Figure 1.4 (b)-(d). As expected, the interferogram of the 2nd IFPI shifted to higher frequencies as temperature decreased. Concurrently, the 1st and 3rd interferogram remained unchanged. No 
crosstalk was observed between IFPIs in this experiment, indicating that this technology holds considerable potential for distributed sensing applications.
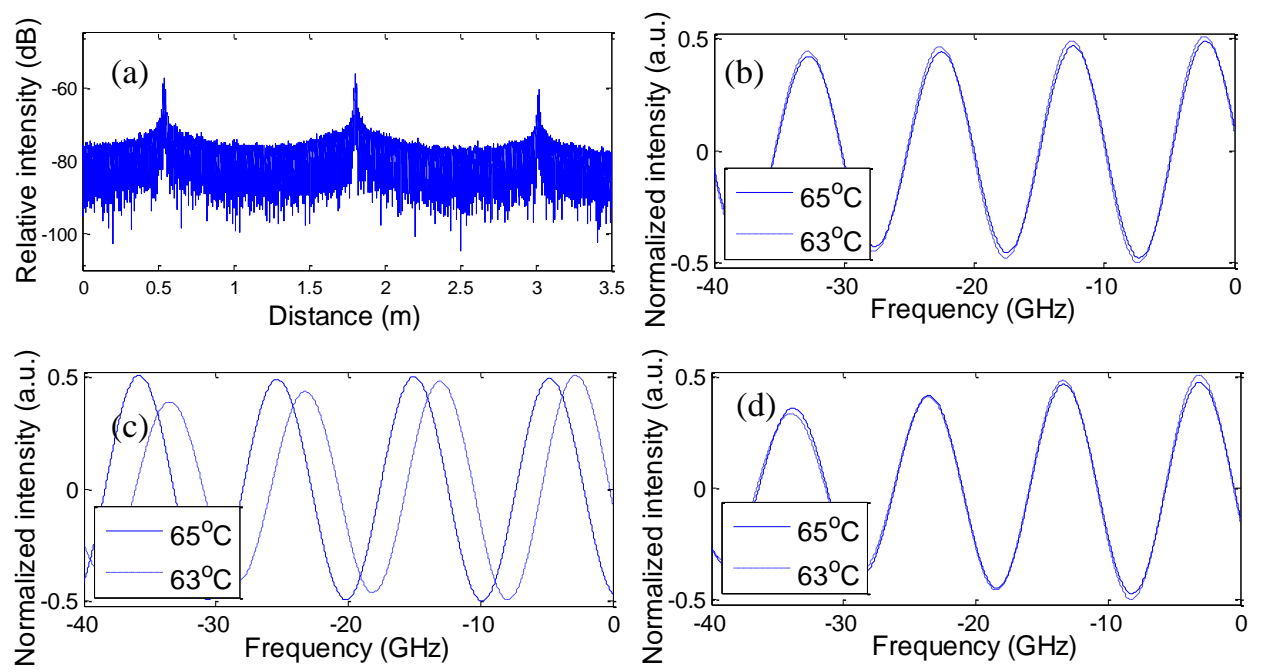

Figure 1.4. Demonstration of ultra-weak IFPIs for distributed sensing, where only the $2^{\text {nd }}$ IFPI is subject to temperature change: (a) reflection distributions of 3 IFPIs; (b) interferogram of $1^{\text {st }}$ IFPI when ambient temperature of $2^{\text {nd }}$ IFPI is varied; (c) interferogram of $2^{\text {st }}$ IFPI at $63^{\circ} \mathrm{C}$ and $65^{\circ} \mathrm{C}$; (d) interferogram of $3^{\text {rd }}$ IFPI when temperature of $2^{\text {nd }}$ IFPI is varied.

A key advantage of an IFPI array over the Rayleigh scattering method is that an IFPI does not require a large bandwidth to resolve small changes with high spatial resolution. To demonstrate this feature, a $1 \mathrm{~cm}$ IFPI was experimentally tested using different laser sweeping bandwidths. Figures 5(a)-(c) depict the interferograms of the IFPI at $63^{\circ} \mathrm{C}$ and $65^{\circ} \mathrm{C}$ with 3 laser sweeping bandwidths. It can be observed that the interferograms generated using these different interrogation bandwidths are similar. The identical temperature response with different sweeping bandwidths, shown in Figure 1.5 (d), further confirms IFPI's capability to operate within relatively narrow bandwidths. 

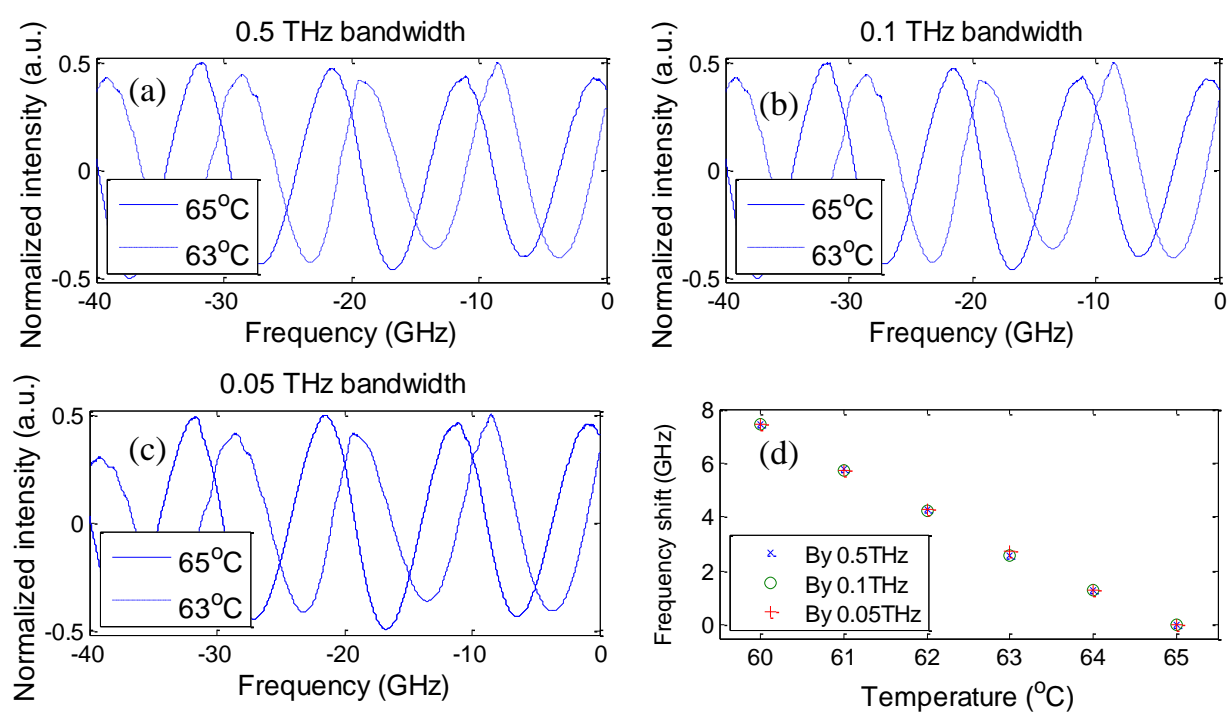

Figure 1.5 Temperature response of a $1 \mathrm{~cm}$ IFPI interrogated using different laser sweeping ranges: (a)-(c) interferogram of the IFPI (d) temperature response with 3 interrogation bandwidths.

To investigate the spatial resolution of the proposed technology, an IFPI array was fabricated along the fiber in a continuous fashion with identical individual cavity length to be $1 \mathrm{~cm}$. The reflection distribution of this array is depicted in Figure 1.6(b), which spans $5 \mathrm{~cm}$. The reflectivity of reflectors in this array is around $-60 \mathrm{~dB}$. The interferograms of all 5 IFPIs were first measured as a reference. Then, a cube of ice was placed $\sim 1 \mathrm{~cm}$ away from the center of the IFPI array as sketched in in Figure 1.6 (a). The interferograms were again taken, and the frequency shifts as a function of IFPI location plotted in Figure 1.6 (c). A Gaussian-like temperature distribution was observed, in which the center IFPI experienced a temperature around $1{ }^{\circ} \mathrm{C}$ lower than IFPIs at either edge as expected. This experiment proves that continuously cascaded IFPIs can be used for distributed sensing. The spatial resolution is the cavity length of the individual IFPIs, i.e., $1 \mathrm{~cm}$ in this test. 

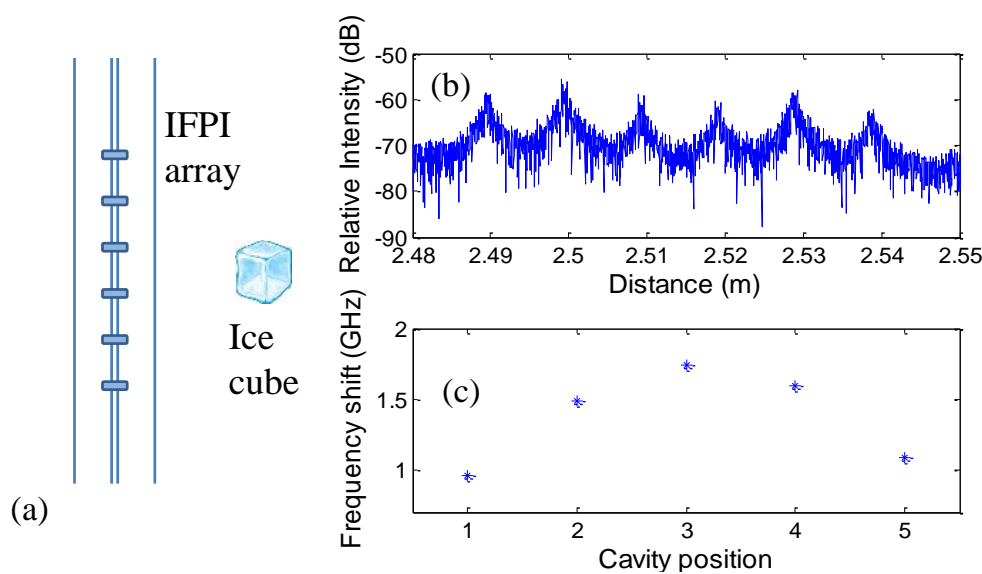

Figure 1.6 Experimental study of continuously cascaded ultra-weak IFPIs: (a) test setup; (b) reflection distribution of 5 IFPIs; (c) frequency distribution measured using IFPI array.

To evaluate system-level accuracy, a stability test was conducted by fixing the ambient condition of a $1 \mathrm{~cm}$ IFPI. 100 groups of interferogram were generated using this device. The frequency shift of each interferogram relative to its initial status was calculated. The standard deviation of the frequency shift is less than $100 \mathrm{MHz}$, determining the system's detection limit. Given the experimentally measured sensitivity of $-1.5 \mathrm{GHz} /{ }^{\circ} \mathrm{C}$, its temperature detection limit is calculated to be less than $0.067{ }^{\circ} \mathrm{C}$.

Previous studies of weak FBG sensors suggest that the multiplexing capacity is mainly limited by two types of crosstalk [6]. The first is spectral shadowing - spectral distortion of the downstream devices caused by the insertion loss of the upstream devices; the second is multiple-reflection crosstalk, or the spectral distortion induced by false signals, which undergo multiple reflections between upstream devices, and experiences the same time delay as the real signal. Both types of crosstalk are exponentially proportional to the reflectivity of the device. Ideally, the reflectivity of an IFPI is calculated by doubling the reflectivity of a single reflector. The smallest reflector achieved in our lab was $-72 \mathrm{~dB}$, converted to a $-69 \mathrm{~dB}$ IFPI, which is more than $30 \mathrm{~dB}$ 
smaller than the current ultraweak FBGs. A theoretical model was applied to estimate the multiplexing capacity of the proposed ultraweak IFPI sensor array [6]. The spectral shadowing effect was simulated in a sensor array of 100,000 IFPIs. In this model, the frequency shifts of all upstream sensors can maximally contribute an unwanted spectral shift of $63.64 \mathrm{MHz}$ to the last IFPI, converted to a reasonable temperature measurement uncertainty of $0.042^{\circ} \mathrm{C}$. In addition, when multiple-reflection crosstalk is considered as the only noise source, a total multiplexing number of 1 million maintains a signal-tonoise ratio (SNR) of $20 \mathrm{~dB}$, indicating its negligible influence. To summarize, 100,000 is approximated as the system's multiplexing capacity. In practice, this number will decrease due to the coherence length limit, total length of fiber, detector quality, and unwanted extra loss along the fiber. Nevertheless, the proposed ultraweak IFPI array technology holds considerable potential to increase the multiplexing capacity by orders of magnitude beyond current methods.

\section{Conclusions}

To conclude, we reported an ultra-weak IFPI array fabricated by fs laser for distributed sensing applications. Reflectivity of weak reflectors decreases as the fs laser power is reduced. IFPI cavities with different physical lengths $(1 \mathrm{~cm}$ and $1 \mathrm{~mm})$ showed identical temperature sensitivity $\left(-1.5 \mathrm{GHz} /{ }^{\circ} \mathrm{C}\right)$. A distributed temperature sensing test was conducted and no crosstalk between IFPI elements observed, implying utility as a distributed sensing system. The system was experimentally proven to be capable of operating within a small bandwidth. A small-scale temperature distribution test was conducted on a continuously cascaded ultra-weak IFPI array, demonstrating its high 
spatial resolution. The temperature detection limit of this system was measured to be less than $0.0667{ }^{\circ} \mathrm{C}$.

This research was sponsored in part by NSF CCF-1439011. 


\section{MANUSCRIPT}

2. Terahertz fiber Bragg grating for distributed sensing

by

Zhen Chen, Lei Yuan, Gerald Hefferman and Tao Wei

published in

Photonics Technology Letters, IEEE, vol. 27, no. 10, pp. 1084 - 1087, May. 2015. 


\begin{abstract}
This letter reports a fiber Bragg grating for distributed sensing applications fabricated using single-mode optical fiber and a femtosecond laser and interrogated in the terahertz range. A theoretical model of device behavior was derived, which agreed well with experimentally observed device behavior. In order to investigate the utility of terahertz fiber Bragg gratings (THz FBGs) as a sensing. modality, temperature tests were conducted. The results demonstrated a sensitivity of $-1.32 \mathrm{GHz} /{ }^{\circ} \mathrm{C}$ and a detection limit of less than $0.0017^{\circ} \mathrm{C}$. A temperature distribution test was also conducted using a $\mathrm{THz}$ FBG, demonstrating its potential as a distributed sensing platform with high spatial resolution. The feasibility of interrogating $\mathrm{THz}$ FBGs using narrow interrogation bandwidths was also experimentally shown.
\end{abstract}

\title{
Introduction
}

The Bragg grating is a mature sensing technique that has been widely used for strain, stress, pressure, and temperature measurement. Through the integration of these periodic structures into a variety of waveguides, the utility of Bragg grating technology has been successfully demonstrated over a broad set of frequency ranges. In the optics domain, incident frequencies in the hundreds of terahertz are routinely used to interrogate fiber Bragg gratings (FBG) [14]. By resolving shifts in the reflected spectra, subtle changes in the parameters of interest can be precisely measured. Similar utility has been demonstrated in the microwave domain (a few gigahertz) through the successful implementation of coaxial cable Bragg gratings (CCBG) fabricated by introducing discontinuities at the centimeter scale $[15,16]$. 
Both FBG and CCBG have demonstrated their utility for large scale, multiplexed sensing applications $[6,17]$. However, these techniques have distinct limitations; the large frequency ranges necessary for interrogation in the optical domain require swept frequency lasers, or a combination of broadband light source and optical spectrum analyzer, with broad ranges (tens of $\mathrm{nm}$, or a few terahertz, at a wavelength of around $1550 \mathrm{~nm}$ ) [18], while the long pitch-length of CCBGs in the microwave domain limits its spatial resolution ( tens of $\mathrm{cm}$ ) for sensing applications.

Terahertz frequency sensing has emerged as a promising method of surmounting the limitations of both the optical and microwave domains. Terahertz frequencies lie between the optical and microwave frequency ranges, which are hundreds of terahertz and tens of gigahertz, respectively. As a consequence of this spectral position, terahertz sensing has the potential to marry the positive qualities of both optical and microwave Bragg grating techniques [19]; as compared to a FBG and CCBG, THz gratings require a narrower interrogation bandwidth (hundreds of gigahertz) and have greater spatial resolution (pitch length $<1 \mathrm{~mm}$ ), respectively.

This possibility has led to recent promising experimental investigation. Zhou et al., using a KrF laser to modify Topas polymer fiber, interrogated the resulting structure using a high frequency vector network analyzer at hundreds of gigahertz [20]. Similarly, Yan et al. used a CO2 laser to modify a step-index polymer fiber, testing the structure using terahertz time-domain spectroscopy at hundreds of gigahertz [21]. Both methods, however, suffer from significant insertion loss as a result of large perturbations of the waveguides, which significantly limits the multiplexing capability of resulting sensors. Additionally, the need to interrogate these sensors using direct terahertz frequency 
modulation requires the use of precision instrumentation at considerable expense due to the high attenuation of the interrogating circuit at $\mathrm{THz}$ frequencies.

In this letter, we report a terahertz fiber Bragg grating (THz FBG) sensing modality with the potential to overcome these engineering limitations. By using heterodyne mixing, a mainstay of microwave photonics, this technique has the potential to lead to both simplified sensor interrogation using narrow interrogation bandwidths and greatlyenhanced distributed sensing capacity with high spatial resolution $[1,13,22,23]$. The interrogation system, fabrication parameters, and sensing utility of $\mathrm{THz}$ FBGs were experimentally studied, and the results presented in this letter.

\section{Operation Mechanism}

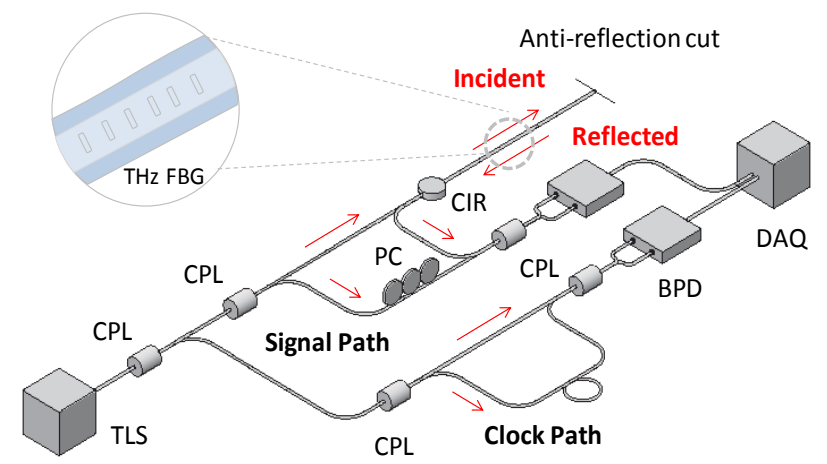

Figure 2.1 Schematic of the interrogation system for THz FBG

The schematic of the interrogation system, based on optical frequency domain reflectometry (OFDR), is shown in Figure $2.1[24,25]$. The light generated by the tunable laser source (TLS) is split by a 90/10 optical coupler (CPL) into two paths, "clock" and "signal". The "clock" path with two 50/50 CPLs is an interferometer that provides the sample clock for the data acquisition card (DAQ), compensating for the non-linearity of the tunable laser. The sampling rate of DAQ is $14 \mathrm{MSa} / \mathrm{sec}$. The light in the "signal" path is split using a 50/50 coupler into a reference arm and a detection arm; a circulator (CIR) guides the reflected light from the THz FBG structure, which is 
terminated with anti-reflected cut; a polarization controller is placed on the reference arm to adjust the interferometer for maximum output; another 50/50 CPL recombines the light from reference arm and detection arm to the balanced photodiodes (BPD) and DAQ. In this setup, the tunable laser sweeps from 1525 to $1555 \mathrm{~nm}$ at $60 \mathrm{~nm} / \mathrm{s}$ scanning speed, corresponding to a total bandwidth of $3.8 \mathrm{THz}$, resulting in 1066357 sampling points in one waveform. The bandwidth of the tunable laser was reduced to $40 \mathrm{GHz}$ to demonstrate the possibility of using smaller bandwidth for sensor demodulation. The real-time system has an update rate of $1.5 \mathrm{sec}$ per round, which includes $0.5 \mathrm{sec}$ for laser scanning and $1 \mathrm{sec}$ for transmission of the data to a PC and calculation. The coherence length of the tunable laser is around $400 \mathrm{~m}$, limiting the total length of the fiber detection arm to be $400 \mathrm{~m}$.

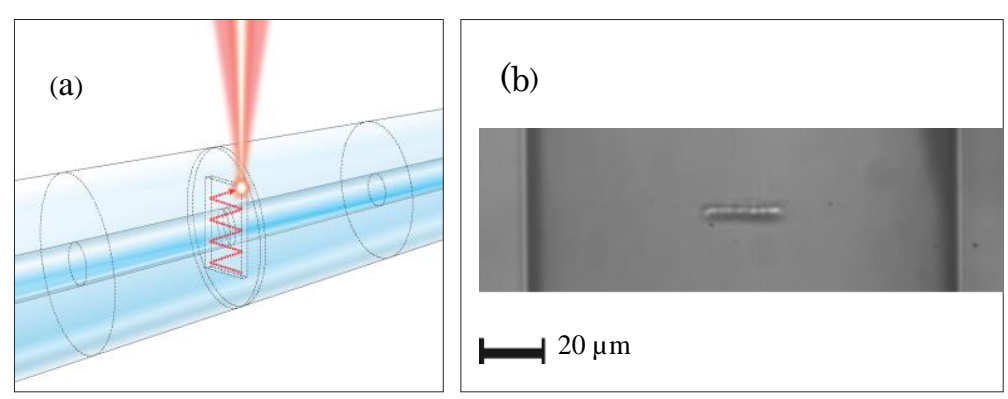

Figure 2.2 Fabrication of THz FBG with femtosecond laser

Figure 2.2 illustrates the technique used to fabricate the THz FBGs under experimental investigation. The structures were fabricated with a Ti:Sapphire fs laser (Coherent, Inc.) micromachining system from the single-mode optical fiber (Corning, SFM-28), with core and cladding diameters of 8.2 and $125 \mu \mathrm{m}$ respectively, for all experimental trials $[10,11,26]$. The insertion of each reflector is smaller than $0.001 \mathrm{~dB}$. 

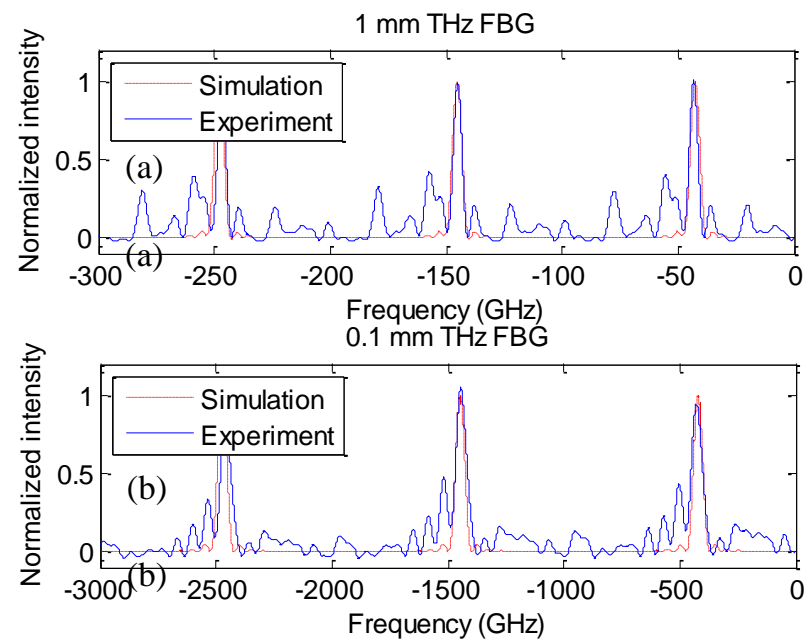

Figure 2.3 Simulation and experimental results: (a) $1 \mathrm{~mm}, 20$ reflection points, $0.11 \mathrm{~W}$; (b) 0.1 $\mathrm{mm}, 20$ reflection points, $0.11 \mathrm{~W}$

A theoretical framework was developed to model the behavior of a multiplexed THz FBG. In this model, M same THz FBGs are embedded along the detection arm, with each FBG containing equally $\mathrm{N}$ reflection points with a period of $\Delta \mathrm{z}$. The total $\mathrm{AC}$ coupled voltage received by the DAQ can then be expressed as:

$$
v_{\text {total }}=2 \eta r I_{\text {ref }} \sum_{m=0}^{M-1} \sum_{n=0}^{N-1} \cos \left[\beta\left(z_{r e f}-z_{m}-2 n \Delta z\right)\right]
$$

where $\eta$ is the light-to-voltage conversion coefficient of the photodiode, $r$ is the reflection coefficient of the FBG reflectors, $\mathrm{I}_{\mathrm{ref}}$ is the light intensity of the reference arm, $\beta$ is the propagation constant, $\mathrm{z}_{\text {ref }}$ is the length from the reference arm to the $P D$, and $z_{m}$ is the length from the start of the $\mathrm{m}^{\text {th }} \mathrm{FBG}$ to the PD. The intensity of the reflected light can be obtained using a Fourier transform. In this study, Rayleigh scattering is defined as the noise floor, resulting in a signal-to-noise ratio (SNR) of $\sim 23 \mathrm{~dB}$. Signal from target FBGs from the fiber under test can be extracted via a Butterworth band-pass filter. The sensing mechanism is based on tracking the frequency shift of the THz FBG signal related with the ambient change. To extract the THz FBG signal shift, a self-mixing technique was applied to the extracted individual THz FBG signal. The reflected signal 
in frequency domain is squared and filtered using a low-pass filter to obtain the FBG reflection spectrum:

$$
S=2 \eta^{2} r^{2} I_{r e f}{ }^{2} \sum_{i=0}^{N-1} \sum_{j=0}^{N-1} \cos [2 \beta(i-j) \Delta z]
$$

Figure 2.3 shows both simulation and experimental results from $1 \mathrm{~mm}$ and $0.1 \mathrm{~mm}$ THz FBGs. Both structures were fabricated with 20 reflection points using a fs laser at $0.11 \mathrm{~W}$ power. Some noise features are evident in the experiment signals, which are attributed to imperfections introduced during fabrication. The simulation results agree well with the experimental data.

\section{Experimental Results and Discussions}

In order to determine the effect of varying the number reflection points on signal quality, the full width at half maximum (FWHM) of signals from gratings with differing numbers of reflectors were measured. Three THz FBGs were fabricated using the same period $(1 \mathrm{~mm})$ and same fabrication power $(0.11 \mathrm{~W})$, and with 10, 20, and 40 reflection points, respectively. Data were sampled 100 times from each THz FBG. The average FWHM results for the 10, 20, and 40 reflection point THz FBGs were $7.03 \mathrm{GHz}, 3.85$ $\mathrm{GHz}$, and $1.27 \mathrm{GHz}$, respectively. These results indicate that, when period and fabrication power are held constant, increasing the number of reflection points of a $\mathrm{THz}$ FBG results in enhanced signal quality factor (Q-factor). However, the trade-off is the mitigated spatial resolution due to the increased grating length.

To investigate the potential utility of a $\mathrm{THz}$ FBG as a temperature sensor, a $\mathrm{THz}$ FBG was fabricated using a fs laser power of $0.11 \mathrm{~W}$, a period length of $1 \mathrm{~mm}$, and 20 reflection points. The THz FBG was placed in a temperature-controlled water bath and 

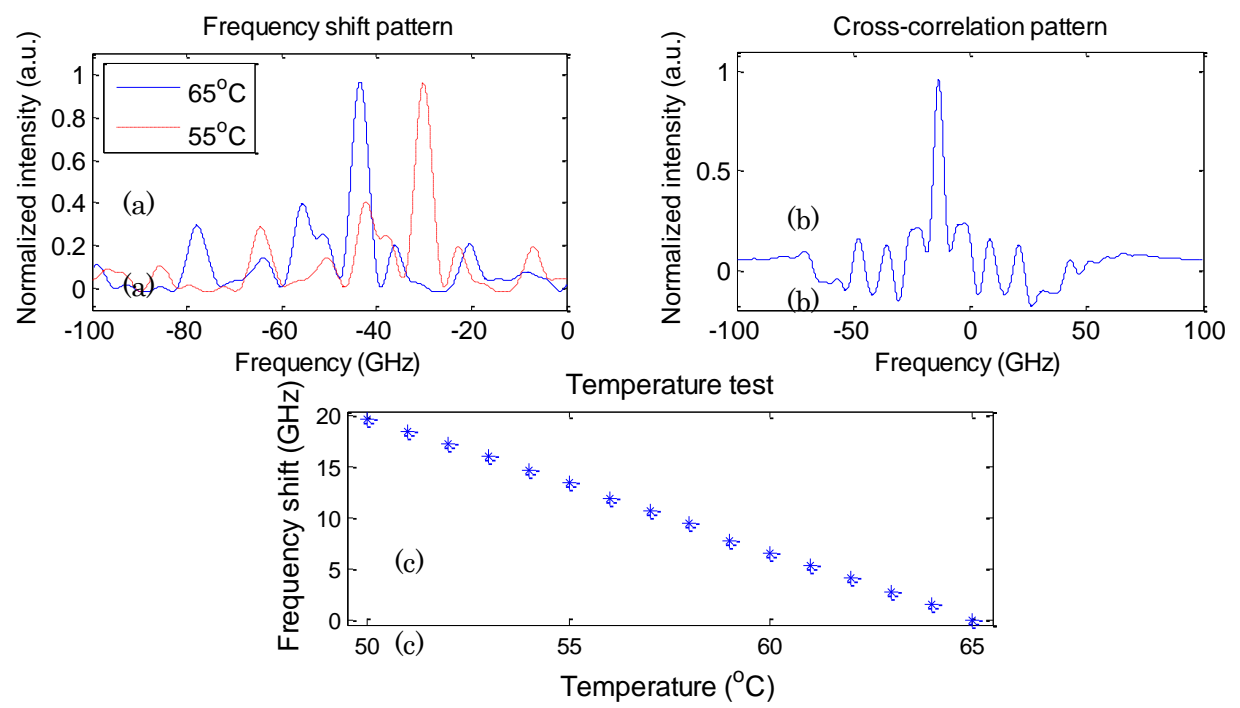

Figure. 2.4. Temperature response: (a) $1 \mathrm{~mm} \mathrm{THz} \mathrm{FBG} \mathrm{reflection} \mathrm{spectra} \mathrm{at} 65^{\circ} \mathrm{C}$ and $55^{\circ} \mathrm{C}$; (b) cross-correlation pattern to extract the frequency shift from (a); (c) frequency shift as a function of temperature

the sensor's temperature response measured. Figure 2.4 (a) shows the THz FBG frequency signal at both $55^{\circ} \mathrm{C}$ and $65^{\circ} \mathrm{C}$. As the temperature increases, the period of FBG increases, causing a corresponding shift in resonant frequency. The frequency shift can be extracted by calculating the cross-correlation pattern. Figure 2.4(b) shows the normalized cross-correlation pattern from Figure 2.4(a) with a frequency shift of 12.84 GHz. Figure 2.4(c) plots the temperature response from $50{ }^{\circ} \mathrm{C}$ to $65^{\circ} \mathrm{C}$. Using this configuration, the temperature sensitivity for the THz FBG was observed to be approximately $-1.32 \mathrm{GHz} /{ }^{\circ} \mathrm{C}$. It is worth noting that the sensitivity of $\mathrm{THz} F B G$ is much larger than conventional microwave grating due to the fact that the interrogation window in the proposed setup is in optical range, and the grating resonant peak under test is at a much higher order in comparison with 1 st order in a microwave grating. For $1 \mathrm{~mm}$ grating, the resonant peaks range from 1923th to 1967th order, given that the laser tuning bandwidth is from 1525 to $1555 \mathrm{~nm}$.

In order to investigate the distributed sensing capability of the system, a 40reflection point THz FBG was fabricated with a period of $1 \mathrm{~mm}$, shown in Figure 5(a). 


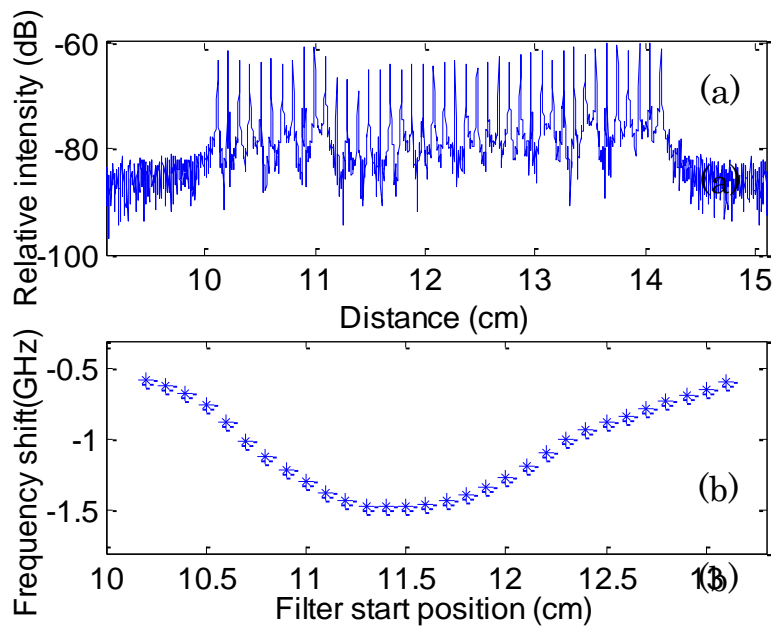

Figure 2.5. Distributed sensing test with THz FBG array: (a); a time-domain 40 reflection point THz FBG signal; (b) frequency-shift distribution measured using THz FBG array.

The system was calibrated using the reflectivity of an APC connector, which was previously measured to be $-60 \mathrm{~dB}$ using precision instrument. The reflectivity of each point is around $-70 \mathrm{~dB}$. The reflectivity of each reflection point varies due to imperfections in the fabrication process. The reflection spectra of THz FBG were first measured with no temperature change as reference. An ice cube was then placed $\sim 1 \mathrm{~cm}$ away from the THz FBG close to center in order to introduce a temperature distribution along the fiber sensor. The spectra were again taken and a high order $0.1 \mathrm{~ns}$ time-domain moving filter, corresponding to $1 \mathrm{~cm}$ in spatial domain, was used to gate the FBG signal with a step of $1 \mathrm{~mm} .90 \%$ of the filter window overlapped with its neighboring filter window. The frequency shift as a function of filter start position was plotted in Figure 2.5 (b). A Gaussian-like temperature distribution was observed in which the center $\mathrm{THz}$ FBG experienced a frequency shift corresponding to a temperature approximately $1{ }^{\circ} \mathrm{C}$ lower than that of either edge of the THz FBG sensor. A real-time experimental demo was videotaped and attached to this letter. This experiment demonstrates that THz FBGs hold the potential for continuous distributed sensing with high spatial resolution. In 

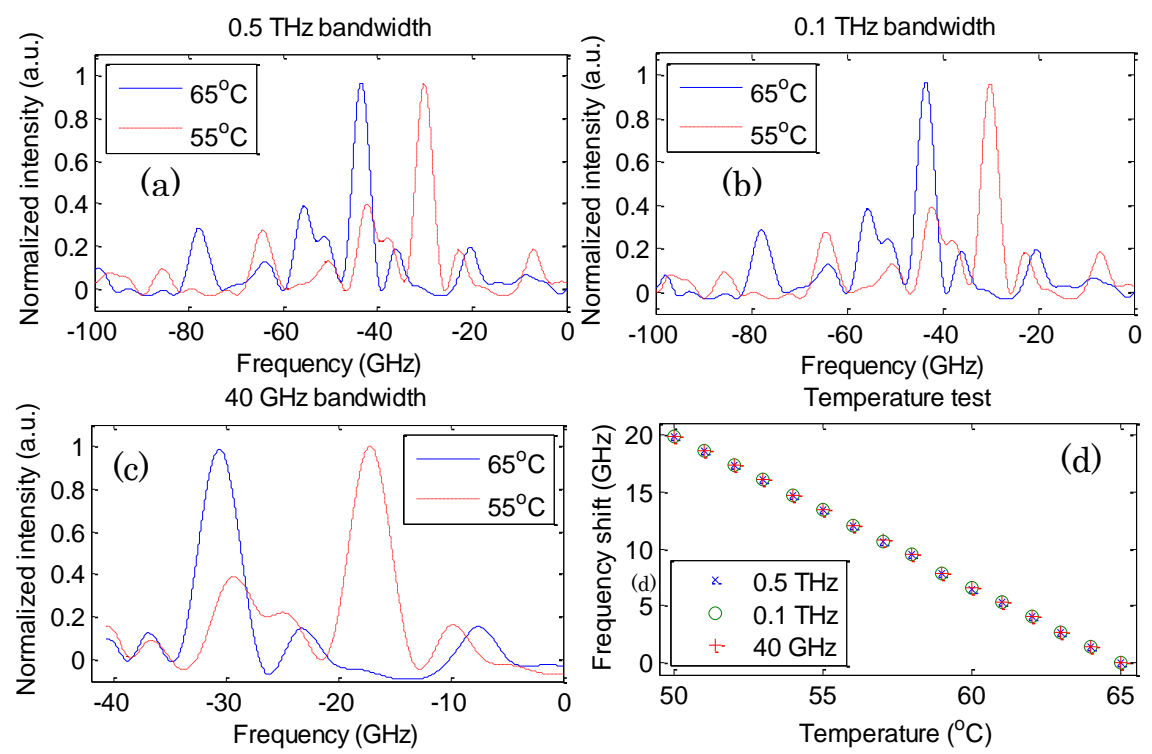

Figure 2.6. Temperature response of $1 \mathrm{~mm}$ THz FBG using different laser sweeping ranges: (a) - (c) temperature response with 3 interrogation bandwidth, (d) temperature test with different interrogation bandwidth

addition, the ultraweak reflection nature of so fabricated THz FBGs promises a huge multiplexing capacity [27].

A key feature of $\mathrm{THz}$ FBGs is that they require a much narrower detection bandwidth than FBGs in the optical frequency range while maintaining good spatial resolution. To demonstrate this feature, a THz FBG with 20 reflection points was tested using differing sweeping bandwidths from a tunable laser. Figure 2.6 (a-c) shows the spectra of the sensor under test using these differing laser sweep bandwidths. Figure 2.6 (d) shows the temperature response for each different bandwidth, which are observed to agree well with each other. These results demonstrate that, when compared with a conventional optical FBG, use of a $\mathrm{THz}$ FBG can effectively reduce detection bandwidth.

To evaluate system-level accuracy, a stability test was conducted by fixing the temperature of a $1 \mathrm{~mm}, 20$ reflection point THz FBG. 100 spectra were recorded using 
this configuration. The frequency shift of each spectrum relative to its initial status was calculated. The standard deviation of the frequency shift was less than 2.27 MHz. Given the experimentally measured sensitivity of $-1.32 \mathrm{GHz} /{ }^{\circ} \mathrm{C}$, its temperature detection limit is calculated to be less than $0.0017^{\circ} \mathrm{C}$. This demonstrates that $\mathrm{THz}$ FBG holds significant potential for high-accuracy detection. The temperature sensing dynamic range is limited by the free spectral range (FSR) of THz FBGs. Frequency shift beyond FSR will result in spectral ambiguity problem. For example, $1 \mathrm{~mm}$ THz FBG has a FSR of $\sim 100 \mathrm{GHz}$, corresponding to a dynamic range of $\sim 76^{\circ} \mathrm{C}$. The dynamic range is inversely proportional to the pitch length of $\mathrm{THz}$ FBG. In addition, large dynamic range requires wide interrogation bandwidth.

\section{Conclusions}

To conclude, this letter reports the development of a FBG operating in the terahertz range with the demonstrated potential to combine the high spatial resolution of FBGs with the narrow detection bandwidth of CCBGs. The effect of varying the number of reflectors on signal quality was quantified. The potential of a THz FBG as a temperature sensor was experimentally validated, with a sensitivity of $-1.32 \mathrm{GHz} /{ }^{\circ} \mathrm{C}$ and a detection resolution of less than $0.0017^{\circ} \mathrm{C}$. The utility of $\mathrm{THz}$ FBGs for distributed sensing with high spatial resolution was experimentally demonstrated. Similarly, differing interrogation bandwidths $(0.5 \mathrm{THz}, 0.1 \mathrm{THz}$, and $40 \mathrm{GHz})$ were shown, in Figure 2.6, to have identical temperature sensitivities, illustrating that $\mathrm{THz}$ FBGs can be successfully interrogated using narrower bandwidths than current optical FBGs. 
3. Ultra-weak waveguide modification with femtosecond laser pulses

by

Zhen Chen, Gerald Hefferman, Lei Yuan, Yang Song and Tao Wei

published in

Photonics Technology Letters, IEEE, vol. 27, no. 16, pp. 1705 - 1708, Jun. 2015. 


\begin{abstract}
This letter reports a new fabrication technique to inscribe in-line, ultra-weak waveguide structures. To the best of the authors' knowledge, this is the first time that an in-line grating structure has been fabricated within the core of an optical fiber with an intact buffer coating, allowing the fiber to retain optimal mechanical properties, an important consideration for strain and temperature sensing applications. Low energy pulses from a femtosecond laser were adjusted to avoid buffer absorption while inscribing a terahertz fiber Bragg grating (THz FBG) within the optical fiber core. Strain and temperature tests were conducted using both a THz FBG sensor fabricated using the reported method and a second THz FBG fabricated using a previously reported method requiring buffer removal. Highly similar results from both Thz FBGs were observed, indicating that the new intact buffer fabrication technique holds substantial potential as a method of fabricating optical fiber grating structures for distributed sensing applications.

\section{Introduction}

Femtosecond laser has been attracting more interests in micromachining [28, 29]. Due to the non-linear absorption, the laser induced breakdown could precisely deliver the high-intensity energy to the focal point causing minimum damage to the surrounding material [30]. The ultra-short pulse could effectively introduce the refraction index change to transparent material. With the modification by femtosecond laser, regular single mode fiber can be used to fabricate the high sensitive optical devices, including Fabry-Perot cavity, fiber Bragg grating, long period grating, substrate/probe for surface enhanced Raman spectroscopy (SERS) detection, etc. [1, 10, 13, 26, 31-38]. 
Recently, terahertz fiber Bragg grating (THz FBG) was successfully fabricated by the femtosecond laser [16]. The pitch length was setup in sub-centimeter scale to achieve the $\mathrm{THz}$ domain detection. Ultra-weak $(<-80 \mathrm{~dB})$ reflectors were periodically seeded along the single mode fiber and no crosstalk was found. The THz FBG holds the great potential for high resolution distributed sensing. Before the fabrication of the $\mathrm{THz}$ FBG, it is necessary to remove the buffer from femtosecond laser processing region due to the different material absorption between the buffer coating and silica glass. However, practically the bare fiber is easy to break with poor mechanical property. It is hard to handle the bare fiber without the coating material during the fabrication process. The THz FBG sample was also fragile during the delivery and testing. This is especially true for long distributed THz FBG arrays. Although it is possible to recoat the bare fiber sensor with UV curable material after the fabrication, the recoating length was limited. Here stresses the engineering challenge: during the femtosecond laser fabrication, can we process the sample without stripping the buffer.

This letter reports a new femtosecond (fs) laser fabrication method to modify the ultra-weak waveguide structure on the THz FBG sensor. The buffer was able to remain on the senor during the fs laser fabrication process, which significantly enhance the mechanical prosperity of the sensor. Experiments were conducted between the $\mathrm{THz}$ FBG with previous fs laser fabrication method and the one we proposed with intact polymer buffer coating. Highly similar results were observed.

\section{Operation Mechanism}

Both grating structures were interrogated using optical frequency domain reflectometry (OFDR) $[17,18]$ and fabricated using a Ti: Sapphire fs laser (Coherent, 
Inc.) micromachining system. Single mode optical fiber (Corning, SMF-28) with core and cladding diameters of 8.2 and $125 \mu \mathrm{m}$, respectively, and a dual acrylate buffer was used in the fabrication of both gratings.

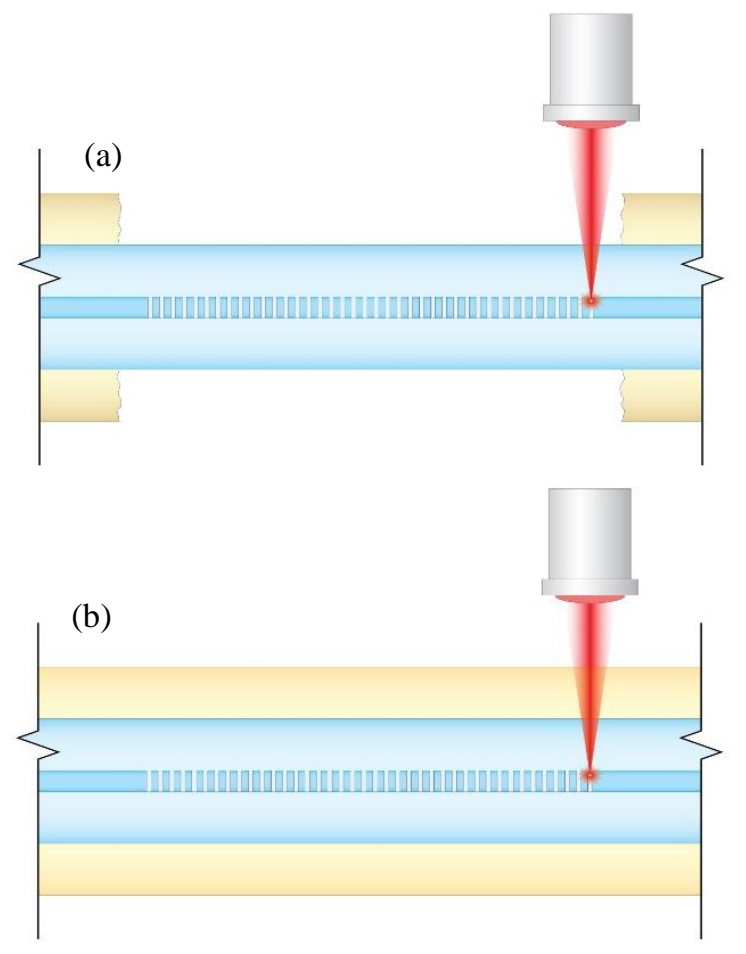

Figure 3.1 Schematic of femtosecond laser fabrication

In the case of the stripped-buffer grating, the laser power used during fabrication was $0.11 \mathrm{~W}$, resulting in the creation of a weak core mode reflection. This laser power was strong enough to be absorbed by the buffer material of the optical fiber to melt and deform the polymer layer, defocus the fs laser beam and fail the grating fabrication. This justifies the necessity to remove the buffer prior to fabrication of the grating structure. Reflection signals recorded using this method were measured to be $22 \mathrm{~dB}$ above the Rayleigh level, which was considered the noise floor in this study, offering good signal quality for detection. Figure 3.2 (a) shows a THz FBG (41 reflectors, or 40 periods, pitch length $1 \mathrm{~mm}$ ) fabricated using this earlier method requiring buffer removal. 
In order to fabricate a similar grating structure in which the buffer layer of the fiber under process was preserved, fs laser power was adjusted to $0.085 \mathrm{~W}$; at this reduced level, the laser energy was not absorbed by the dual acrylate coating at a level sufficient to melt the fiber buffer. It is worth noting that the melting power of buffer materials under this setup is $0.05 \mathrm{~W}$ when laser beam is focused on polymer coating. The power $0.085 \mathrm{~W}$ used in grating fabrication here is focused on the fiber core, explaining that it is $70 \%$ higher than the threshold power to melt polymer. While core reflectors were still inscribed along the length of the fiber under process, these perturbations within the optical fiber core were reduced to the point of being invisible using conventional light microscopy. Signals recorded from these reflectors were measured to be $10 \mathrm{~dB}$ above the noise floor, again sufficient for good signal quality. Figure 3.2 (b) shows a THz FBG (41 reflectors, pitch length $1 \mathrm{~mm}$ ) fabricated using this proposed method. No insertion loss was found.
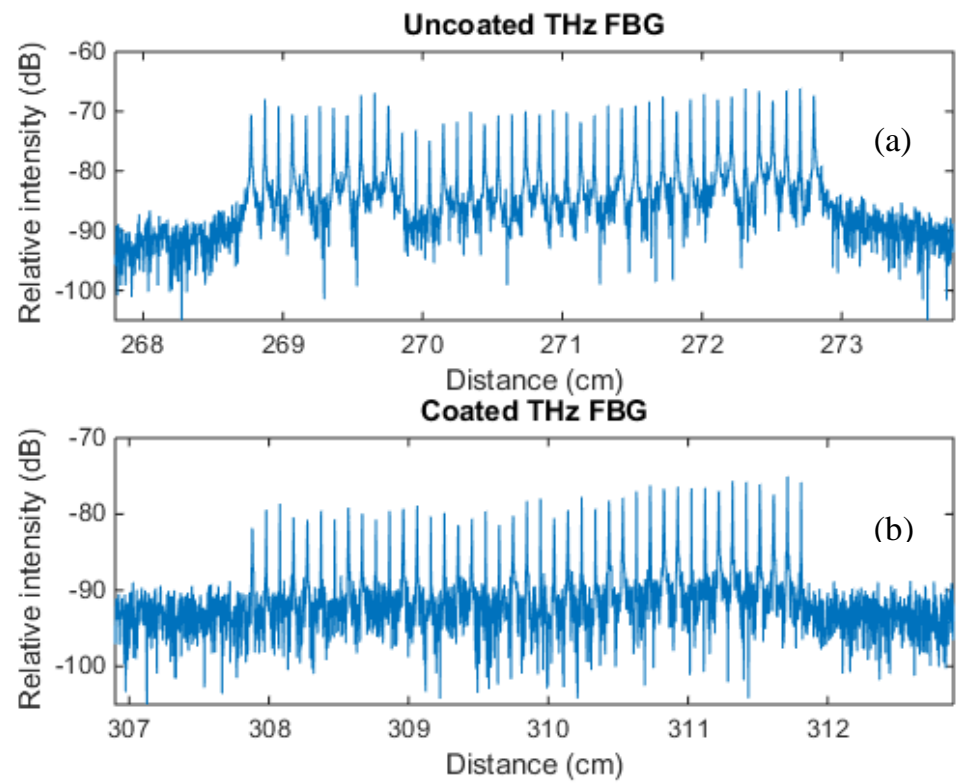

Figure 3.2 Distance domain signals for THz FBG fabricated by femtosecond laser: (a) with buffer coating stripped; (b) proposed method with buffer

\section{Experimental Results and Discussions}


To investigate the utility of the sensor constructed using an intact buffer, both the sensors in Figure 3.2 were interrogated in series and subjected to identical strain and temperature testing for direct comparison.

In order to conduct a strain test, one end of the optical fiber under test housing both grating structures was secured to an optical bench with the other end free to hang. Weights were sequentially added to the free end of the fiber in $3 \mathrm{~g}$ intervals; in total, 30 $\mathrm{g}$ were added to the free end of the fiber under test. The results of this strain test for both grating structures are shown in Figure 3.3 (a). A linear relationship was found between the accumulated screw weight and frequency shift, with an R2 values for both grating structures greater than 0.9998 and slopes of $1.8695 \mathrm{GHz} / \mathrm{g}$ and $1.7993 \mathrm{GHz} / \mathrm{g}$ for the uncoated and buffer-coated gratings, respectively. The slight discrepancy between these two slopes is surmised to be attributable to the difference in effective Young's modulus between the two sensors, the result of mechanical differences between with or without the impact buffer coating.
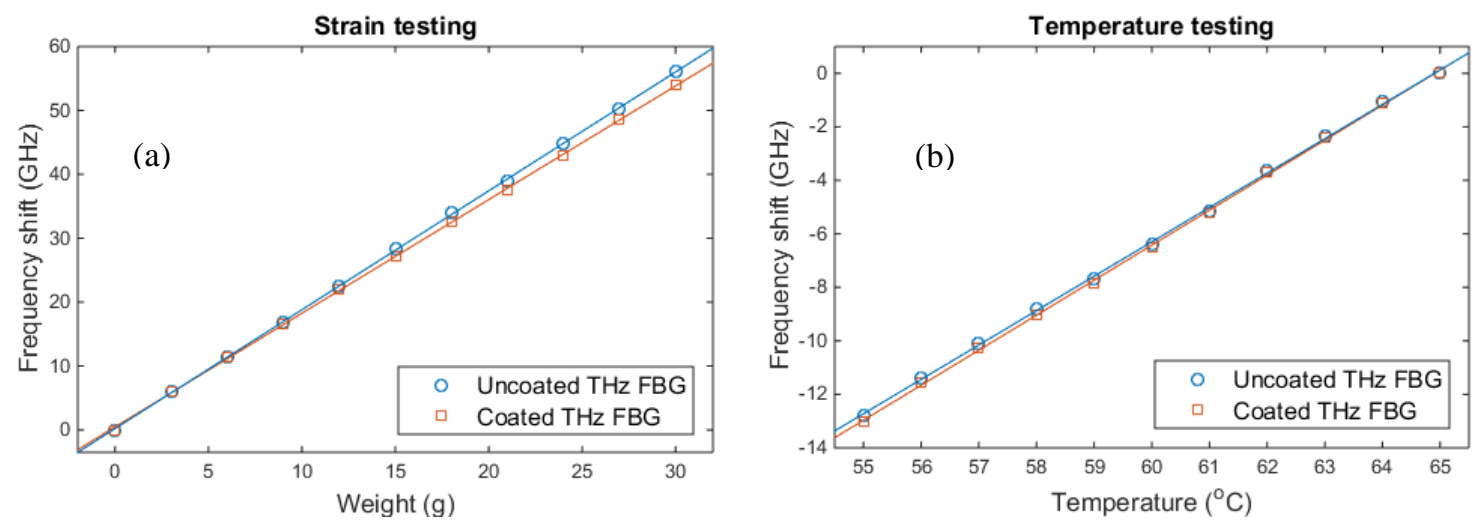

Figure 3.3 Strain and temperature test with two different THz FBGs: (a) strain test results; (b) temperature test results

Similarly, to the strain testing set-up, temperature sensing using the two gratings was conducted by simultaneously interrogating the two sensors in series with both 
sensors submerged within a water bath. The water was first heated to $65^{\circ} \mathrm{C}$, and then cooled to $55^{\circ} \mathrm{C}$. The test results are for both grating structures shown in Figure 3.3 (b). A liner relationship was found between the temperature drop and the sensor frequency shift, with R2 values for both gratings greater than 0.9994 and trend line slopes of 1.2791 $\mathrm{GHz} /{ }^{\circ} \mathrm{C}$ and $-1.3041 \mathrm{GHz} /{ }^{\circ} \mathrm{C}$ for the uncoated and buffer-coated gratings, respectively. As the heat capacity of water is larger than the buffer coating material, the sensor with the intact buffer coating cooled slightly faster than the grating without the buffer coating, which is surmised to account for the slight difference between the twotemperature testing result trend line slopes.

The results of both strain and temperature testing demonstrate that the fiber grating structure with an intact buffer coating has analogous sensing capabilities to the uncoated grating structure fabricated using conventional methods.

\section{Conclusions}

To conclude, this letter reports a new femtosecond laser fabrication method to modify the ultra-weak waveguide structures. To our best of knowledge, this is the first time to process the fiber sensing device without stripping the buffer. The fs laser output power was carefully adjusted to avoid the buffer coating melt and deformation, which enables grating fabrication with intact buffer, and largely enhances the sensor mechanical property. Strain and temperature test were conducted on both the uncoated buffer THz FBG sensor and the coated one. Highly identical results were observed, indicating that the proposed method holds great potential for distributed sensing measurement. 
4. Phase-shifted terahertz fiber Bragg grating for strain sensing with large dynamic range

by

Gerald Hefferman, Zhen Chen and Tao Wei

published in

Photonics Technology Letters, IEEE, vol. 27, no. 15, pp. 1649 - 1652, Jun. 2015. 


\begin{abstract}
Recent advances in optical fiber sensing techniques have demonstrated the utility of terahertz (THz) gratings as a modality for strain and temperature sensing. However, these techniques remain reliant on the use of higher-order resonant peaks, enhancing their sensitivity at the cost of limited dynamic range. The use of a lower-order resonant peak for sensing can lead to a larger dynamic range at the cost of accuracy. This letter reports a $\pi$-phase-shifted THz fiber Bragg grating, fabricated using a femtosecond laser, capable of detecting changes in strain over a substantially larger dynamic range than previously reported methods with improved accuracy. A second $\mathrm{THz}$ grating without a $\pi$-phase-shifted structure, but otherwise identically constructed, was interrogated in series on the same optical fiber. The two devices were simultaneously experimentally investigated using a strain test $(\sim 1.0 \mathrm{~m} \varepsilon$ in total), and the results presented in this letter. Additionally, theoretical models of the devices were created, which closely matched experimentally observed device physics.
\end{abstract}

\title{
Introduction
}

Optical fiber sensors have a unique set of characteristics that make them particularly useful as strain and temperature sensors [23, 26, 34-36, 39-41]. Their chemical stability and immunity to electromagnetic interference make them ideally suited to sensing in harsh environments, while the ease with which multiple sensors can be fabricated in series and simultaneously interrogated allows for multiplexed sensing across considerable distances $(\sim \mathrm{km})$, making optical fiber sensors a viable solution in structural health monitoring, energy, and aerospace applications [1, 13, 42, 43].

Fiber Bragg gratings in particular have demonstrated their utility in these areas, and 
are a fundamental element of many optical fiber sensing techniques. Recently, fiber Bragg gratings fabricated with grating structures corresponding to the terahertz range (THz FBGs) have demonstrated additional characteristics that make them particularly well-suited to applications requiring large-scale, multiplexed sensing techniques [24, 44]. Chief among these advantages are their narrow interrogation bandwidth (hundreds of gigahertz) and their high spatial resolution $(<1 \mathrm{~mm})$.

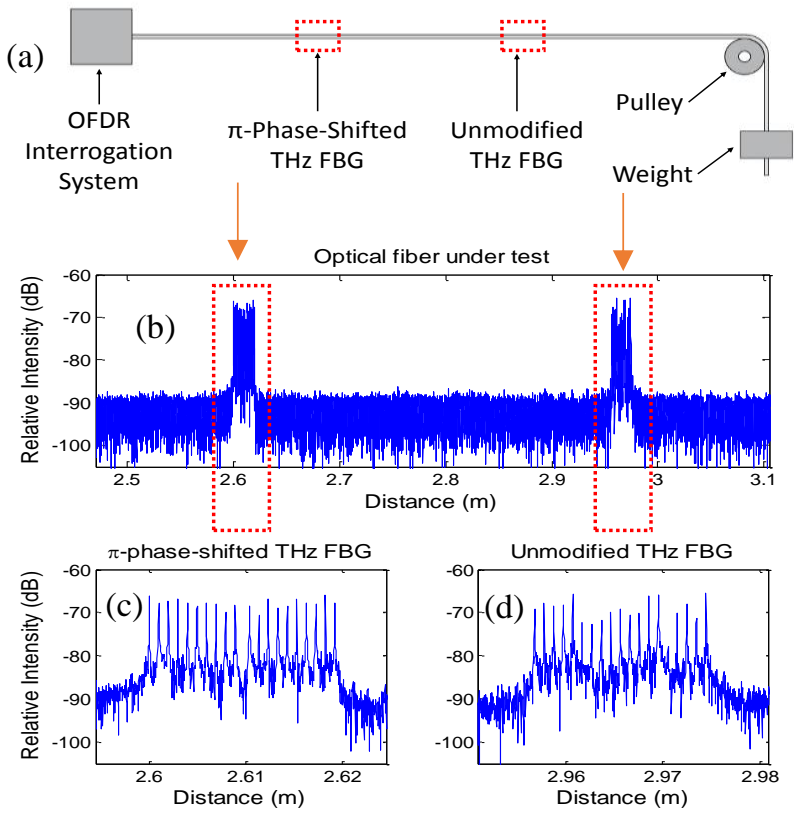

Figure 4.1 Experimental set-up and time-domain plots of the optical fiber under test. (a) Diagram of the strain testing set-up; (b) time domain plot of optical fiber with both the $\pi$-phase-shifted and unmodified THz FBGs in series; (c) detail of the time-domain trace at the area of the $\pi$-phaseshifted THz FBG; (d) detail of the time-domain trace at the area of the unmodified THz FBG.

Despite these advantages, however, current THz FBG sensing techniques remain limited by their relatively narrow dynamic range. This limitation is chiefly due to the use of higher-order resonant peaks ( $1923^{\text {rd }}$ to $1967^{\text {th }}$ order with a pitch length of $1 \mathrm{~mm}$ ) for parameter measurement and signal processing, which reduces dynamic range [44]. This trade-off is particularly limiting in the case of structural health monitoring, an area in which the need for structural data gathered during episodes of high strain and 
temperature is particularly acute. On the other hand, the use of lower-order resonance peaks at lower frequencies can resolve this ambiguity problem at the cost of decreased sensitivity.

It is known that the quality factor of the resonance peak can be significantly improved by introducing a defect into periodic structures, such as photonic crystals or FBGs $[20,45,46]$. This letter reports a grating structure interrogated in the terahertz range that surmounts this limitation. By interrogating the structure using lower-order $\left(9^{\text {th }}\right.$ order) resonance peaks and introducing a $\pi$-phase-shift into a THz FBG structure, the dynamic range of the resulting sensor was substantially increased and the corresponding loss of accuracy minimized, respectively. Experimental investigation of the $\pi$-phase-shifted THz FBG was conducted and compared against a THz FBG that was not phase-shifted, but was otherwise identically fabricated and interrogated in series along the same optical fiber. The results of this investigation, as well as a theoretical simulation modeling device physics, are presented in this letter.

\section{Operation Mechanism}

Optical frequency domain reflectometry (OFDR) was used as the interrogating system in this study [47]. The theoretical framework used to model the $\pi$-phase-shifted THz FBG is as follows: $M \pi$-phase-shifted THz FBGs are embedded along the detection arm of the system, with each $\pi$-phase-shifted FBG containing $N$ reflectors ( $N$ is even) and a pitch length $\Delta z$. The total AC coupled voltage received by the DAQ can then be expressed as: 


$$
\begin{aligned}
v_{\text {total }}=2 \eta I_{\text {ref }} & \sum_{m=0}^{M-1}\left\{\sum_{n=0}^{N / 2^{-1}} \cos \left[\beta\left(z_{\text {ref }}-z_{m}-2 n \Delta z\right)\right]\right. \\
& \left.+\sum_{n=N / 2}^{N-1} \cos \left[\beta\left(z_{r e f}-z_{m}-2 n \Delta z\right)\right]\right\}
\end{aligned}
$$

where $\eta$ is the light-to-voltage conversion coefficient of the photodiode, $r$ is the reflection coefficient of the FBG reflectors, $I_{r e f}$ is the light intensity of the reference arm, $\beta$ is the propagation constant, $z_{\text {ref }}$ is the length from the reference arm to the photodiode (PD), and $z m$ is the length from the start of the $m^{\text {th }}$ grating to the PD. The intensity of the reflected light can be obtained using an inverse Fourier transform. In this study, Rayleigh scattering is defined as the noise floor, resulting in a signal-to noise ratio (SNR) of approximately $25 \mathrm{~dB}$. Signals from a target FBG along the fiber under test can be extracted via a band-pass filter. The sensing mechanism is based on tracking the frequency shift of the THz FBG signal, which is related to ambient change - in this case, strain along the fiber under test.

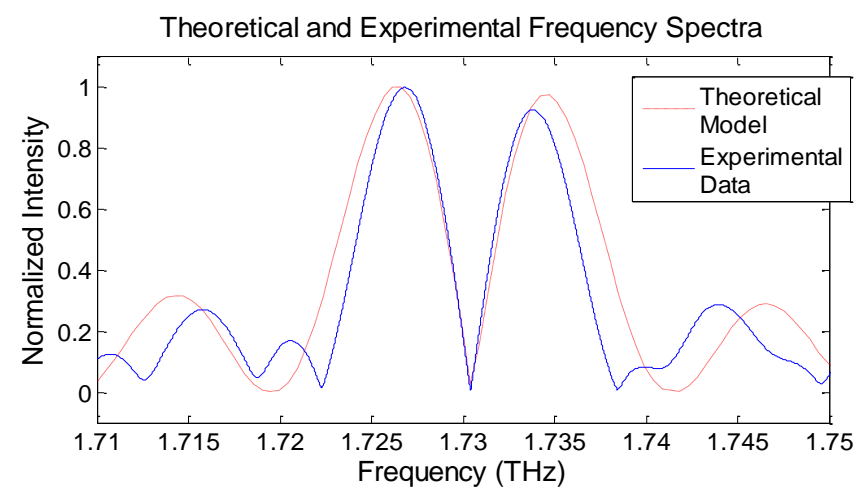

Figure 4.2 Simulation results and experimentally collected spectrum of the $\pi$-phase-shifted $\mathrm{THz}$

Data from the $\pi$-phase-shifted THz FBG is extracted from the total $M$ FBGs using a Butterworth band-pass filter; data from the unmodified $\mathrm{THz}$ FBG is similarly 
extracted. By applying a Fourier transform and discarding the phase information gathered by OFDR, the time domain signals of both gratings can be found, as shown in Fig. 1 (a-c). As the number of detection points is cut by half using this method, the signal bandwidth is $1.85 \mathrm{THz}$. Applying a second Fourier transform on the resulting time domain scanning signal yields a frequency domain periodic signal in the $\mathrm{THz}$ range.

Fig. 2 shows theoretically-modeled and experimentally observed frequency domain signals generated using this method. Simulated data was generated using a pitch-length of $0.9825 \mathrm{~mm}$, a reflectivity of $10^{-6}$, and a bandwidth of $3.8 \mathrm{THz}$. Peak shift in this signal is related to strain change along the length of the fiber under test. The $9^{\text {th }}$ order resonance peak of the frequency domain signal was used in this test.

\section{Experimental Results and Discussions}

In order to experimentally investigate the sensing utility of the $\pi$-phase-shifted fiber Bragg grating, a strain test was conducted. A Ti:Sapphire femtosecond laser (Coherent, Inc.) was used to fabricate both gratings within single-mode optical fiber (Corning, SMF-28) at a laser power of $0.11 \mathrm{~W}[37,38]$. Each grating contains 18 reflection cavities with a pitch-length of $1 \mathrm{~mm}$. A $\pi$-phase-shift (1.5 times the pitch length) was added halfway along the length of the phase-shifted THz FBG.

Both the $\pi$-phase-shifted and unmodified THz FBGs were tested in series and interrogated simultaneously. The optical fiber with both sensors was secured to an optical bench on one end, with the other end free to hang. Weights were sequentially added to the free end of the optical fiber, and the resulting output from each sensor was recorded using the method described above. In all, 20 weights, with a total mass of 124 
$\mathrm{g}$, were added to the free end of the fiber at $6.2 \mathrm{~g}$ intervals. In order to quantify the strain change along the optical fiber generated by the addition of each set of weights, the unmodified THz FBG was interrogated using higher order modes, as described in previous work [44]. Due to the fact that the sequential addition of each weight to the fiber under test resulted in an incremental phase shift of less than $2 \pi$ radians, this method provided a direct measure of strain along the fiber. Importantly, however, the total change after the addition of all the weights resulted in a combined shift greater than $2 \pi$ radians using higher-order modes, which is beyond the detection limit of the earlier approach, but within the limit of the method described in this letter. Using this technique, total strain change of $1.0 \mathrm{~m} \varepsilon$ in intervals of approximately $71.5 \mu \varepsilon$ was measured over the course of the experimental trial.
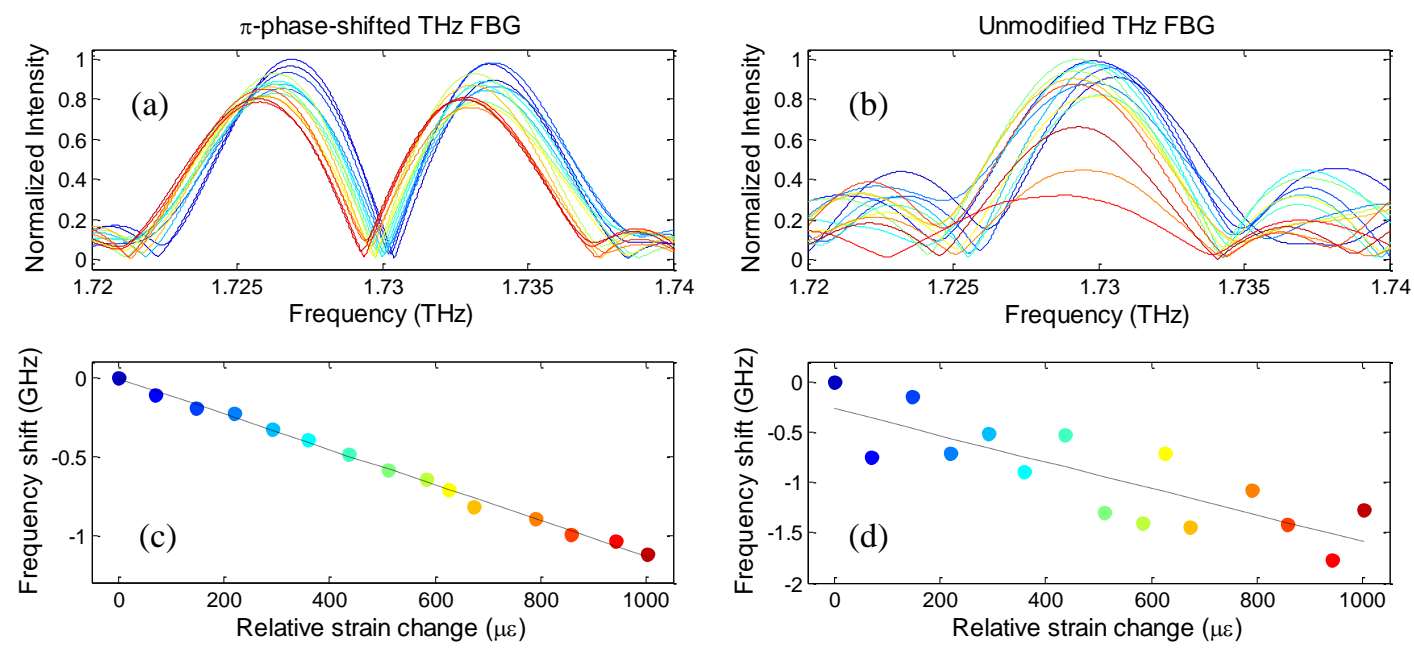

Figure 4.3 Experimental results of strain testing along the optical fiber under test. (a) Spectra of $\pi$ phase-shifted THz FBG; (b) spectra of unmodified THz FBG; (c) relative peak shift of the $\pi$-phaseshifted THz FBG as a function of strain and best-fit trend line; (d) relative peak shift of the unmodified THz FBG as a function of strain and best-fit trend line. Points and curves of the same color were collected and calculated simultaneously as weights were sequentially added to the fiber under test.

As both sensors were placed along the same optical fiber and tested simultaneously, crosstalk between the two sensors was considered. By using ultraweak reflectors in the 
fabrication of both grating structures $(-60$ to $-70 \mathrm{~dB})$, crosstalk between the two $\mathrm{THz}$ FBGs was effectively minimized [24]

The results of this experiment are shown in Fig. 3. The comparison between the spectra of $\pi$-phase-shifted THz FBG and unmodified THz FBG clearly showed that the quality factor of the resonance peak/dip improves by introducing a $\pi$-phase-shift into the normal THz FBG. As a result, the $\pi$-phase-shifted THz FBG demonstrated much improved linearity between strain change and frequency shift, with a $\mathrm{R}^{2}$ value of

0.9962 and a trend-line slope of $-0.0011 \mathrm{GHz} / \mu \varepsilon$. In contrast, data collected from the unmodified THz FBG demonstrated less linearity, with an $\mathrm{R}^{2}$ value of 0.6699 . These results demonstrate that the $\pi$-phase-shifted THz FBG performed substantially better than the unmodified THz FBG in the detection of strain changes along the length of the optical fiber under test. In addition, as observed in the experiments, the intensity of reflection spectrum decreases as the loaded strain increases. This is attributed to the fact that the refractive index contrast, or reflectivity, of the fs modified reflectors decreases under strain. Importantly, the experimental results also showed that the signal of the proposed THz FBG was sufficiently strong for strain sensing within the testing range up to $1 \mathrm{~m} \varepsilon$.

\section{Conclusions}

To conclude, this letter demonstrates that the application of a $\pi$-phase-shift to $\mathrm{THz}$ FBGs provides the enhanced accuracy necessary to use lower-order resonance peaks for sensor interrogation, allowing for expanded dynamic range relative to the previously reported method using higher-order resonance peaks. This sensing strategy was theoretically-modeled and experimentally investigated using both a $\pi$-phase-shifted 
THz FBG and an unmodified THz FBG in series along the same optical fiber under test. The resulting linearity of the peak shift of the $\pi$-phase-shifted THz FBG $\left(\mathrm{R}^{2}=0.9962\right)$ demonstrates that this technique has the potential to lead to the application of THz FBGs to domains in which large dynamic range is a critical engineering requirement. Structural health monitoring provides an example of one such area; by allowing for enhanced real-time monitoring of critical infrastructure, THz FBGs with enhanced dynamic range have considerable potential societal benefit. 
5. Terahertz-range interrogated grating-based two-axis optical fiber inclinometer

by

Zhen Chen, Gerald Hefferman, Lei Yuan, Yang Song and Tao Wei

published in

Optical Engineering, SPIE, vol. 55, no. 2, pp. 026106, Feb. 2016. 


\section{Abstract}

This manuscript reports a two-axis fiber inclinometer fabricated using an ultraweak terahertz-range fiber Bragg grating (THz FBG). Three sensing grating structures were inscribed along a single-mode optical fiber using a femtosecond laser, bound together into a sensing array using thermoformed plastic, and fixed to a two-axis rotation stage. Inclination tests were performed in which the fiber was deflected from $0^{\circ}$ to $1.7^{\circ}$. These tests were repeated at eight azimuthal angles in increments of $45^{\circ}$ (from $0^{\circ}$ to $315^{\circ}$ ). The standard deviation of the largest inclination angle error was $0.048^{\circ}$ and the stability of the inclination angle was $0.030^{\circ}$.

\section{Introduction}

Optical fibers have seen increasing use as sensing elements for strain, stress, temperature and refractive index measurements in a wide variety of engineering applications. A more recent application of fiber sensors is to determine angle and position information used for three-dimensional shape sensing. Due to its compact size and ability to conform to relatively complex shapes, optical fiber can be easily fixed along an object of interest to mirror its orientation. Multiple strain sensors can then be integrated together to form a single sensing probe aligned to the changing contour along the object. By using a multi-core fiber or a multi-fiber bundle packaging method, three or more strain sensors can be packaged in parallel together as one sensing element to measure strain information in three dimensions, data that can ultimately be used to find the angle and position information necessary for three-dimensional shape sensing.

Different fiber distributed strain sensors have been proposed and commercialized to reach this engineering goal. The two main research areas undergirding this 
technology are fiber Bragg gratings (FBG) and coherent optical frequency domain reflectometry (C-OFDR). Both methods use differing fundamental physics to reach the same goal; distributed strain sensing along an optical fiber.

A fiber Bragg grating (FBG) is a wavelength-based fiber sensor $[14,48]$. Periodic reflectors are inscribed along a single mode fiber by UV laser to form a wavelengthspecific dielectric mirror that reflects a certain wavelength and transmits the rest. Multiple FBGs can be integrated along one sensing probe using wavelength multiplexing to achieve distributed strain measurements. Miller et al. have proposed several FBG-based structures used to resolve the spatial angle information using multiple FBGs, which include multi-core fibers $[49,50]$ and three-fiber bundles [51] as two-axis fiber inclinometers. More recently, researchers have demonstrated using a femtosecond laser to directly inscribe FBG waveguide structures into a single coreless fiber [52] and standard single mode fiber [53] for three-dimensional shape sensing applications.

Coherent optical frequency domain reflectometry (C-OFDR) $[2,5,54-59]$ is an alternative, state-of-the-art distributed sensing technology. By sweeping the optical frequency with a tunable laser, the Rayleigh backscatter profile along an optical fiber can be measured, which correlates to strain along the fiber under test. This interferometric measurement is capable of maintaining a terahertz-level detection bandwidth with high spatial resolution. Using three or more probes, C-OFDR has successfully demonstrated its ability to deliver three-dimensional shape sensing using both the multicore fiber [60-62] and multiple fiber packing methods [63]. 
Recently, the use of terahertz fiber Bragg grating (THz FBG), an extension of COFDR interrogation, has been demonstrated [44, 64]. A high-power femtosecond laser is employed to inscribe ultra-weak periodic reflectors $(<-70 \mathrm{~dB})$ within the core of a standard single mode optical fiber, providing distributed interferometric strain measurements along the fiber under test and allowing the system to achieve enhanced sensitivity compared with traditional Rayleigh scattering-based $(<-80 \mathrm{~dB})$ C-OFDR techniques. By combining three or more of these THz FBG sensors into a single fiber sensor bundle, the system is able to determine the spatial angle information necessary to act as a two-axis optical fiber inclinometer, an important step towards threedimensional shape sensing.

This manuscript reports a terahertz fiber grating-based two-axis optical fiber inclinometer fabricated using ultra-weak reflection arrays $(-70 \mathrm{~dB})$. Three identical $\mathrm{THz}$ FBGs were aligned and packaged as a single fiber bundle. The differing strain distributions across the three THz FBGs within the sensing probe were measured and used to determine the spatial angle information along the fiber bundle. The inclinometer was tested at eight azimuthal angles (from $0^{\circ}$ to $315^{\circ}$ ). The standard deviation of the greatest inclination angle error was $0.048^{\circ}$ and the inclination angle stability was $0.030^{\circ}$. No cross-talk was found between the ultra-weak reflection arrays.

\section{Operation Mechanism}

In order to experimentally investigate the terahertz grating-based inclinometer concept, a $30 \mathrm{~mm}$-long inclinometer was constructed. Three identical THz FBGs were fabricated with intact buffer coatings using single mode fiber (SMF-28, Corning) and a Ti: Sapphire fs laser (Coherent, Inc.) micromachining system [11, 24, 34, 38, 65]. Each 
sensing array contained 41 ultra-weak reflectors $(\sim-75 \mathrm{~dB})$ with a pitch length of $1 \mathrm{~mm}$. To bind the modified fibers into a three-fiber bundle, the three SMF fibers with $\mathrm{THz}$ FBG arrays were secured equidistant to one another, as shown in Figure 5.2 (a). The three modified fibers were aligned using reference marks $\sim 5 \mathrm{~cm}$ beyond the length of the sensing arrays. Two $40 \mathrm{~mm}$ heat melt tubes (EVA) and a $90 \mathrm{~mm}$ heat shrink tube were placed outside the aligned sensing arrays, as illustrated in Figure 5.1 (a). Hot air $\left(\sim 300 \mathrm{~F}^{\circ}\right)$ was blown from $\sim 1 \mathrm{~cm}$ away and swept across the length of the bundle at a speed of $\sim 1 \mathrm{~cm} / \mathrm{s}$. After subsequent cooling, the three THz FBG sensing arrays were firmly fixed in place, shown in Figure 5.1 (b).

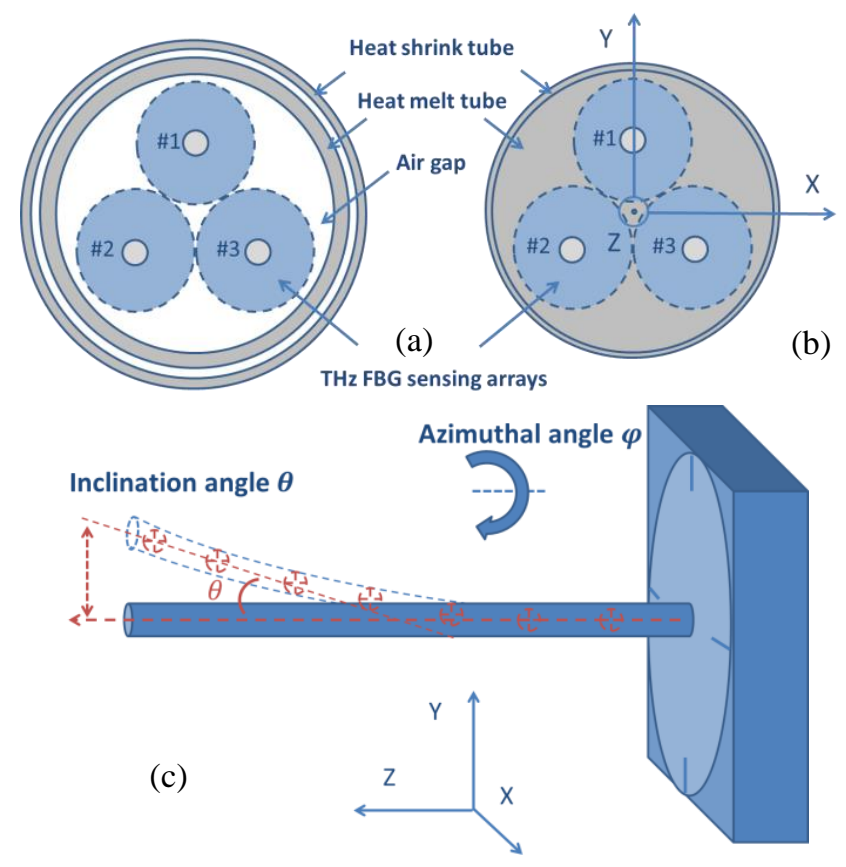

Figure 5.1 Three-core fiber inclinometer bundle packaging and assembling: (a) before heated, (b) after heated, (c) schematic of the assembling the fiber inclinometer

To assemble the inclinometer, one end of the packaged bundle was positioned using a screwed ferrule into the center of a $360^{\circ}$ rotational mount (Newport Inc.) along the $\mathrm{Z}$ axis to give azimuthal rotation control, as illustrated in Figure 5.1 (b). The other end 
was affixed to a micrometer-level stage that could be moved vertically in the $\mathrm{Y}$ direction, enabling inclination angle control.
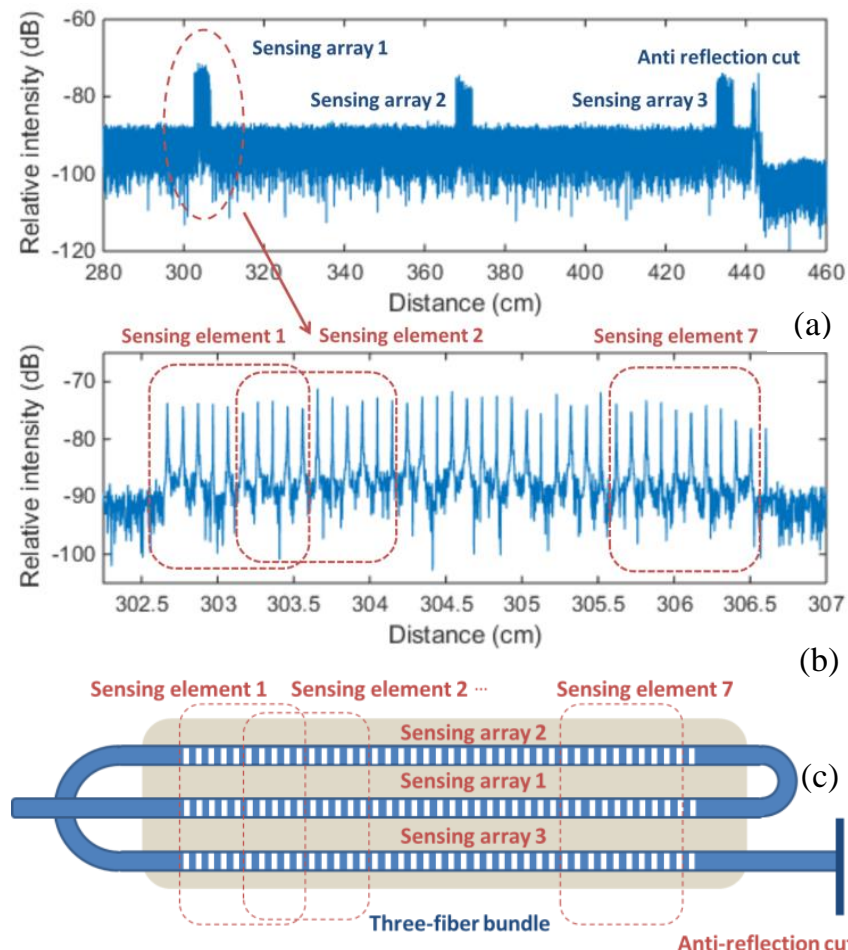

Figure 5.2 Schematic of cascaded THz FBG sensors: (a) the time domain interrogation signal of three identical THz FBG sensing arrays, (b) the details of sensing array 1, (c) the packaged inclinometer bundle

To conduct the inclination measurement experiment, three THz FBG sensing arrays were cascaded in-line, as shown in Figure 5.2, and interrogated via coherent optical frequency domain reflectometry (C-OFDR). For each sensing array, a $10 \mathrm{~mm}$ timedomain moving filter was applied to measure the distributed strain along sensor; in total, 7 strain measurements at $5 \mathrm{~mm}$ steps were extracted. The three strain sensing points at the same position along the inclinometer bundle then form a single sensing element; in total, 7 sensing elements along the $30 \mathrm{~mm}$ sensor were used. Each sensing element contains three-dimensional strain information, which can be used to calculate the spatial position in the three-dimensional Cartesian coordinate system with Frenet-Serret 
formulae (16). The azimuthal angle $\varphi$ was defined as the sensor rotation along the $\mathrm{Z}$ axis. As the sensor was deflected towards the $\mathrm{Y}+$ direction, the inclination angle $\theta$ at a certain azimuthal angle $\varphi$, shown in Figure 5.3 (b), was defined as the acute angle between the $\mathrm{Z}$ axis and line passing through the 6th and 7th sensing elements.

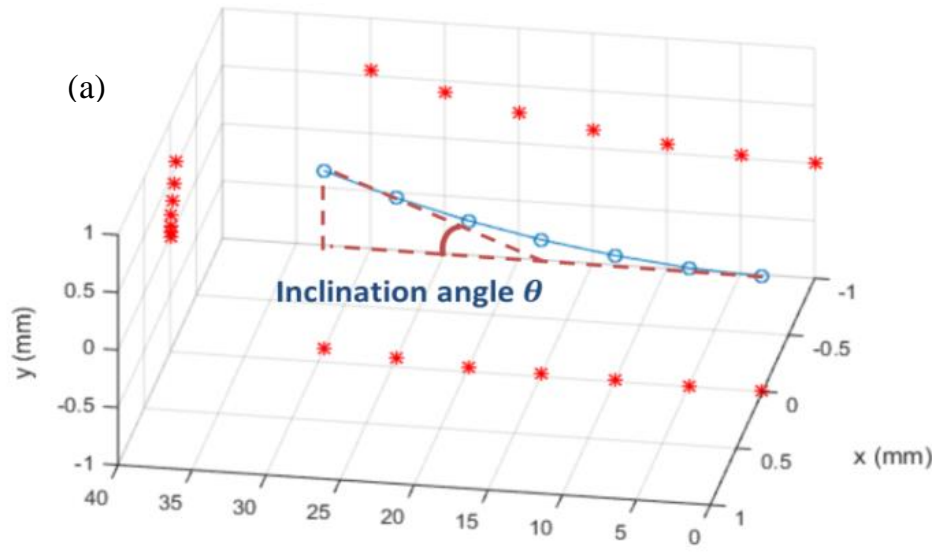

(b)

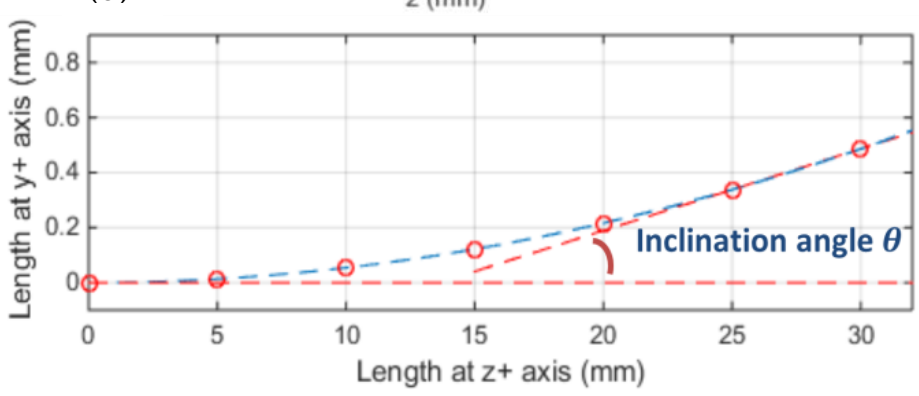

Figure 5.3 Schematic of the measured inclination angle: (a) 3D representation, (b) 2D representation (project to $\mathrm{YZ}$ plane)

In order to account for packaging inconsistencies such as sensing array misalignment or heat shrink tube nonuniformity, calibration must be performed. First the cross-plane of each sensing element, the relative positions of the three strain sensing points were calibrated. As illustrated in Figure 5.4 (d), the bundle was bent towards three different azimuthal angles $\varphi_{1}, \varphi_{2}$, and $\varphi_{3}$, where sensing arrays \#1, \#2, and \#3 were each positioned on top, respectively. The differing strain distributions for the seven sensing points were then measured along each sensing array. For each sensing element, a two-dimensional cross-plane was extracted to calculate the relative position of the 
three strain sensing points. At each sensing element, the balanced boundary condition can be expressed as:

$$
\frac{\varepsilon_{i 1}}{d_{i 1}}=\frac{\varepsilon_{i 2}}{d_{i 2}}=\frac{\varepsilon_{i 3}}{d_{i 3}}
$$
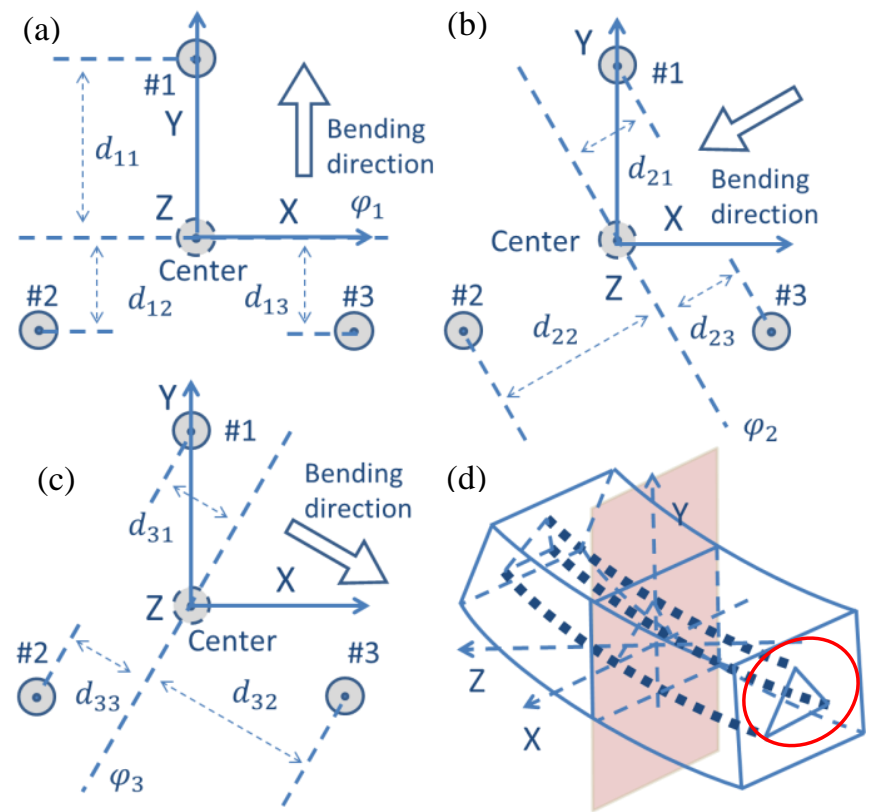

Figure 5.4 Calibrate the relative position: (a) - (c): the cross-plane illustration of bending with sensing array \#1, \#2 and \#3 on top respectively, (d) schematic of inclination bending

where $\varepsilon_{i j}$ is the strain measurement with the $i$ th sensing array on top (at azimuthal angle $\left.\varphi_{\mathrm{i}}\right)$ for the $j$ th sensing array and $d_{i j}$ is the related length of lever arm. It is assumed that the bending is occurring across the center of the sensor at origin $(0,0)$ in the twodimensional plane. The line perpendicular to the bending direction and passing the origin $(0,0)$, can be expressed as:

$$
A_{i} x-y=0
$$

where $A_{i}$ is the slope of the line with ith sensing array on top. At a certain sensing element cross-plane, the relative position of ith sensing array on top at the jth sensing array can be expressed as $\left(x_{i j}, y_{i j}\right)$.The related length of lever arm $d_{i j}$ shown in Figure 5.4 (a) - (c), can be expressed as: 


$$
d_{i j}=\frac{A_{i} x_{i j}-y_{i j}}{\sqrt{{A_{i}^{2}+1}^{2}}}
$$

The initial condition is defined where the relative distance between the sensing points along sensing array \#1 to the $\mathrm{X}$-axis is normalized to 1 . The relative positions of the three sensing points for each sensing element cross-plane were then solved, shown in Figure 5.5 These coefficients were converted to polar form and applied to solve the Frenet-Serret equations.

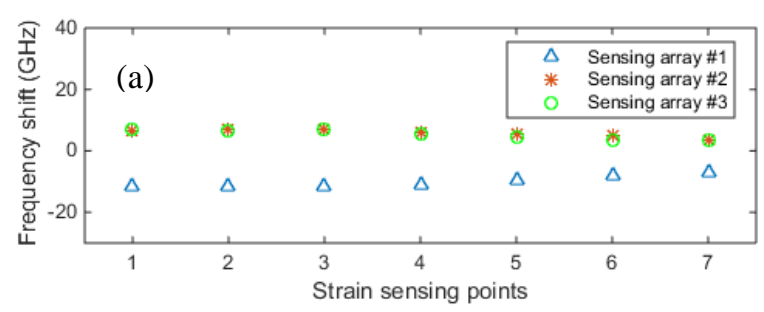

(b)

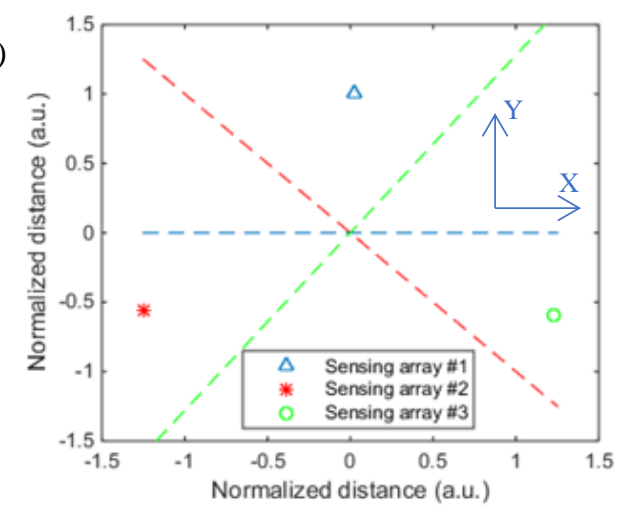

Figure 5.5 Calibrate the relative position: (a) with sensing array \#1 on top, the strain measurement result along the three sensing arrays, (b) the solved relative position on the $1^{\text {st }}$ sensing element cross-plane

Second, the absolute radius can be calibrated based on the assumption that all seven strain sensing points along the sensing array \#1 share the same distance to the center of the inclinometer bundle. Based on the first calibration results, another radius coefficient is set to match the calculated position and the absolute deflection by the Y-directional stage at each azimuthal angle.

\section{Experimental Results and Discussions}


To investigate the utility of the fiber inclinometer, inclination angles from $0^{\circ}$ to $1.7^{\circ}$ with a step of $0.085^{\circ}$ were investigated for all eight azimuthal angles $\left(0^{\circ}, 45^{\circ}, 90^{\circ}\right.$, $135^{\circ}, 180^{\circ}, 225^{\circ}, 270^{\circ}$ and $315^{\circ}$ ). Some of the representative measurement data are plotted in Figure 5.6. The calibration coefficients were implemented before inclination testing. The inclination test results at azimuthal angle $0^{\circ}$ and $270^{\circ}$ are shown in Figure
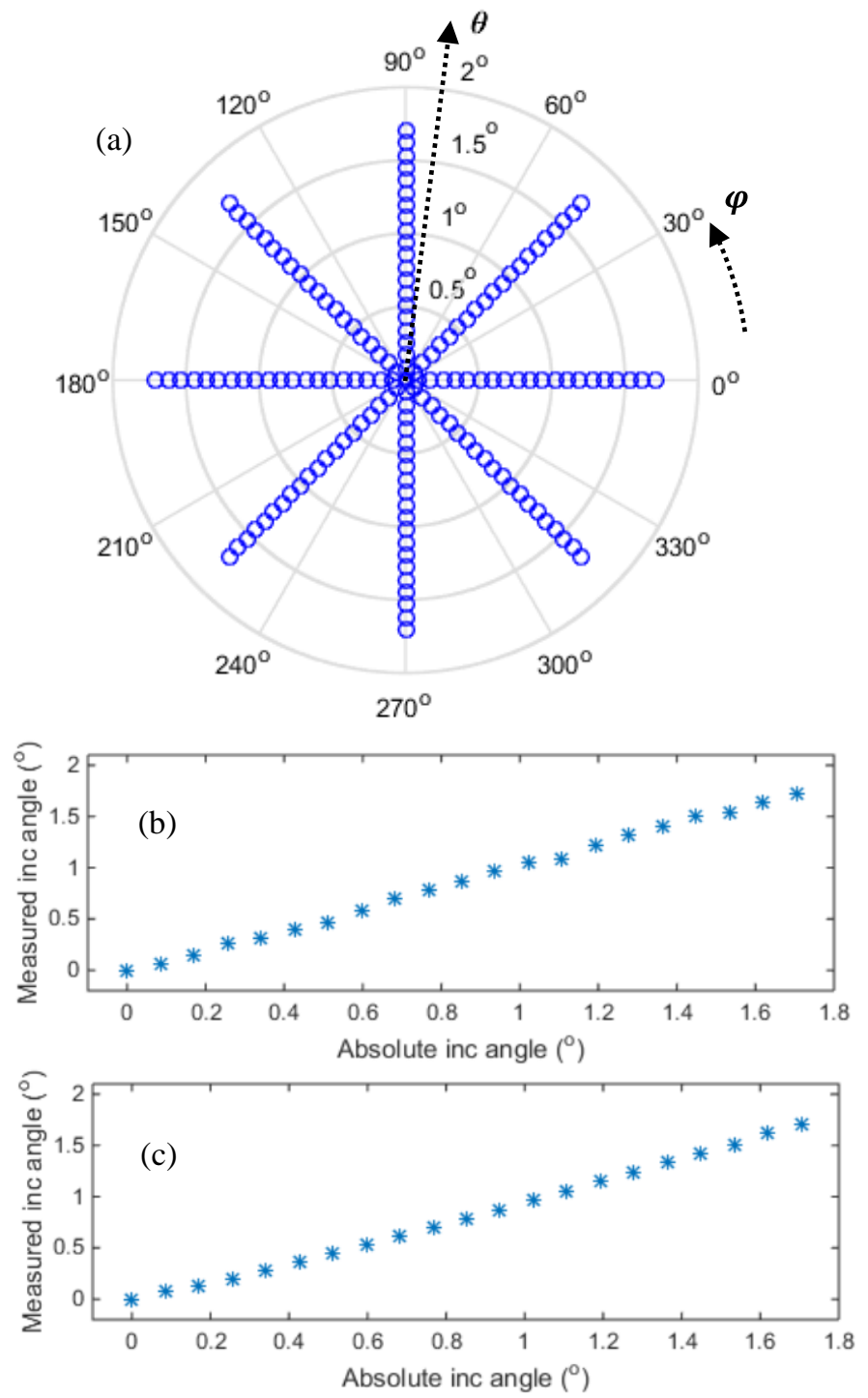

Figure 5.6 Two-axis inclination measurement results: (a) measured inclination angles $\theta=0^{\circ}$, $0.767^{\circ}$ and $1.70^{\circ}$, respectively, at eight differing azimuthal angles $(\varphi)\left(45^{\circ}\right.$ apart from $0^{\circ}$ to $315^{\circ}$ ) (b) measured inclination angle against calculated angle at $\varphi=0^{\circ}$, (c) measured inclination angle against calculated angle at $\varphi=270^{\circ}$ 
5.6 (b), (c). Similar results were found with other azimuthal angles. The largest error standard deviation was $0.048^{\circ}$.

The stability test of the proposed inclinometer was conducted with a fixed $0^{\circ}$ azimuthal angle and $0^{\circ}$ inclination angle. In total 250 sets of tests were repeated to measure this initial inclination angle. The standard deviation of the measured angle was $0.030^{\circ}$, indicating a good repeatability of this device.

The maximum inclination measurement range was $2.5^{\circ}$ with steps of $0.085^{\circ}$ and the error standard deviation was $0.036^{\circ}$. In these tests, the inclination measurement range was limited by the maximum frequency shift within 1 period of the implemented THz FBG, which is $100 \mathrm{GHz}$ for $1 \mathrm{~mm}$ pitch length.

\section{Conclusions}

To conclude, this manuscript reports a new two-axis optical fiber inclinometer based on terahertz fiber Bragg grating (THz FBG) structures. Inclination angles from $0^{\circ}$ to $1.7^{\circ}$ were tested at eight azimuthal angles (from $0^{\circ}$ to $315^{\circ}$ ), covering one full rotation. The standard deviation of the largest inclination angle error was $0.048^{\circ}$ and the observed inclination angle stability was $0.030^{\circ}$. In addition, the 3-dimensional spatial positions for all sensing elements were solved, indicating this sensor holds substantial potential for three-dimensional distributed sensing. 
6. A low bandwidth DFB laser-based interrogator for terahertz-range fiber Bragg grating sensors

by

Zhen Chen, Gerald Hefferman and Tao Wei

published in

Photonics Technology Letters, IEEE, vol. 29, no. 4, pp. 365 - 368, Feb. 2017. 


\begin{abstract}
This letter reports an all-electronic, low bandwidth swept frequency laser used to interrogate terahertz-range fiber Bragg gratings (THz FBGs) for distributed strain sensing applications. A distributed feedback (DFB) laser with current injection modulation was employed as the swept frequency laser source. Using the resulting narrow bandwidth $(\sim 110 \mathrm{GHz})$ laser frequency sweep, high accuracy distributed strain measurements were achieved. In order to experimentally investigate this concept, a strain test was conducted using terahertz-range fiber Bragg gratings. During the test, the laser sweeping time was limited to less than $2 \mathrm{~ms}$. Highly linear results were found $\left(\mathrm{R}^{2}\right.$ $=0.996)$, with an observed sensitivity of $-0.142 \mathrm{GHz} / \mu \varepsilon$ and the standard deviation of $1.167 \mu \varepsilon$. A multiplexing test was also conducted, and no cross-talk found between sensor elements. These results demonstrate that this interrogation system holds substantial potential as a method of rapid distributed optical fiber frequency-domain sensing.
\end{abstract}

\title{
Introduction
}

Terahertz fiber Bragg gratings (THz FBGs) have been investigated as sensor elements for a number of distributed strain and temperature sensing applications [44]. Femtosecond laser micromachining techniques have been used to fabricate these millimeter-range weak periodic structures within the cores of otherwise unmodified communications-grade optical fibers [26]. The reflectivity of individual reflector elements can be controlled by adjusting the power of the femtosecond laser during fabrication, allowing the system to balance both signal-to-noise ratio and multiplexing capability. High accuracy measurement can be achieved by interrogating the sub- 
terahertz range resonation periods of these THz FBGs at higher orders using an optical frequency sweep. More recently, low interrogation bandwidth $(100 \mathrm{GHz})$ measurements have been effectively demonstrated using $1 \mathrm{~mm}$ pitch length THz FBGs.

Until recently, the interrogation systems used to probe $\mathrm{THz}$ FBGs have all been based on coherent optical frequency domain reflectometry (C-OFDR) $[5,54,66]$, where a key component of the system is a highly coherent swept laser source. Previously, an external cavity laser was employed as the frequency sweep source, which allowed for the interrogation of THz FBGs using a broad sweeping bandwidth $(\sim 3.7 \mathrm{THz})$. When compared with the $100 \mathrm{GHz}$ resonation period of the $1 \mathrm{~mm}$ pitch length $\mathrm{THz}$ FBG, however, this broad sweeping bandwidth may be unnecessary. External cavity lasers require the use of moving mechanical components in order to achieve a frequency sweep, limiting the update rate and increasing the system complexity. This combination of a broader-than-necessary bandwidth and slow update rate has led to increasing interest in the use of alternative swept laser interrogator techniques.

Several other highly coherent frequency sweep sources have been experimentally explored for use with C-OFDR systems; these include temperature and piezoelectrically tuned Nd: YAG ring lasers [67, 68], chirped distributed feedback (DFB) lasers [69] and piezo-electrically tuned fiber grating lasers [70]. Among these sources, the chirp-DFB laser represents a particularly promising candidate. The highly coherent optical frequency output of these lasers can be directly controlled by modulating the injection current. As this frequency modulation technique requires no mechanical movement of the laser cavity, the sweep time can be shortened, potentially increasing the update rate of the interrogation system. Additionally, a sweeping bandwidth of 100 
$\mathrm{GHz}$ can be achieved, a suitable range for the interrogation of $\mathrm{THz}$ FBG sensors with a pitch length of $1 \mathrm{~mm}$ or greater. This letter reports a low bandwidth chirp-DFB laserbased interrogator for THz FBGs. A DFB laser with injection current modulation was used as the frequency sweep source to interrogate two THz FBG sensor elements.

This letter reports a low bandwidth chirp-DFB laser-based interrogator for $\mathrm{THz}$ FBGs. A DFB laser with injection current modulation was used as the frequency sweep source to interrogate two THz FBG sensor elements. An interrogation bandwidth of 110 $\mathrm{GHz}$ within $1 \mathrm{~ms}$ was achieved using the system. In order to experimentally investigate the system, a strain test was performed; highly linear results $\left(\mathrm{R}^{2}=0.996\right)$ were observed, with a sensitivity of $-0.142 \mathrm{GHz} / \mu \varepsilon$ and a standard deviation of $1.167 \mu \varepsilon$. A multiplexing test was also conducted, and no cross-talk observed between the sensor elements. These results demonstrate that this system holds substantial potential as a method of dynamic distributed strain sensing.

\section{Operation Mechanism}

A schematic of the interrogation system, based on coherent optical frequency domain reflectometry (C-OFDR) [25, 64, 71], is shown in Figure 6.1. An arbitrary wave

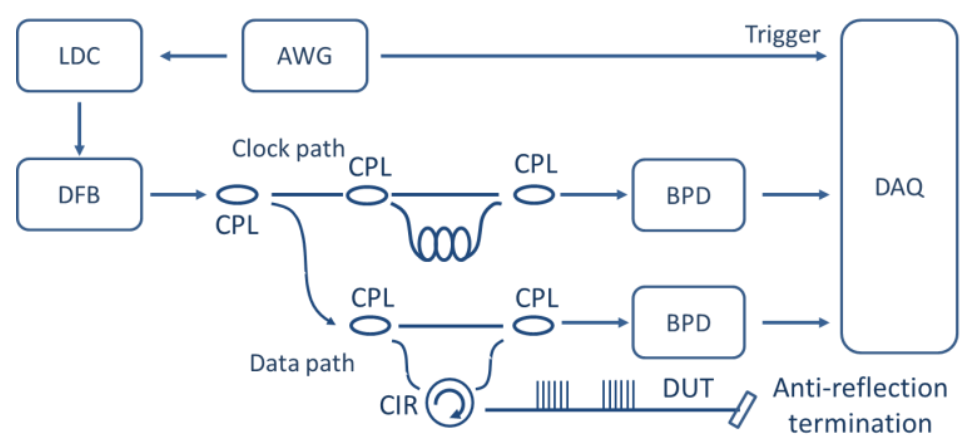

Figure 6.1 Schematic of the chirp-DFB laser-based C-OFDR interrogation system (LDC: laser diode controller; AWG: arbitrary wave generator; DAQ: data acquisition; DFB: distributed feed-back laser; CPL: coupler; CIR: circulator; DUT: devices under test; BPD: balanced photodiode). 

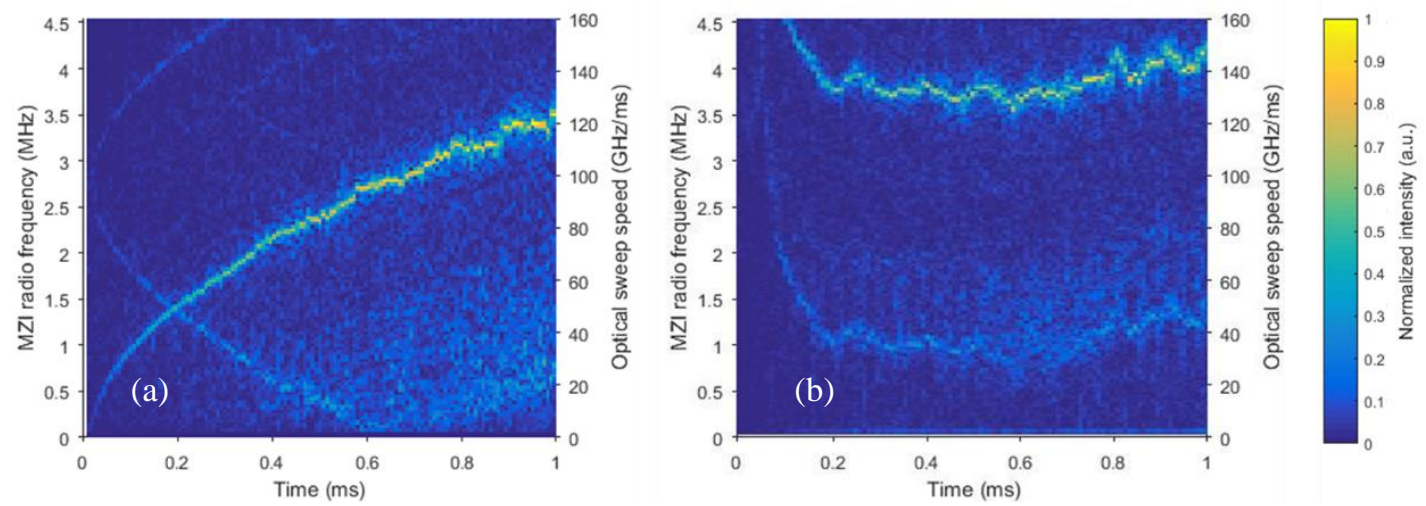

Figure 6.2 The measured MZI radio frequency, or the chirp-DFB laser sweeping speed as a function of time using different voltage modulation waveforms: (a) an initial ramp input; (b) after 6 feedback pre-distortion iterations.

generator $(\mathrm{AWG})$ is used to modulate the DFB laser via a laser diode controller (LDC).

The resulting output of the DFB laser source is sent into the optical system via a 90/10 coupler (CPL), with $90 \%$ of the power directed into the detection path and $10 \%$ of the power directed into the clock path. The clock path is constructed using a Mach-Zehnder interferometer (MZI), with a constant delay length of 54.02 ns. The data path is constructed using two 3-dB couplers and an optical circulator (CIR). The reflected signals from devices under test (DUT) can be measured via this interferometry structure. An anti-reflection termination is used to prevent strong reflections form the end of the DUT. Two balanced photo diodes and a data acquisition card (DAQ) are used to record the resulting interferometric signals. The DAQ has a sampling rate of $130 \mathrm{MSa} / \mathrm{s}$ and 15-bit resolution, and is triggered by synchronization output from the AWG.

Inspired by the work of Satyan et al. [72], an iteration pre-distortion method is applied in order to increase the linearity of the laser sweep. A chirp-DFB sweep model can be established in which the laser output optical frequency can be expressed as:

$$
\omega(t)=\omega_{0}+K_{L}(i) \cdot K_{0} \cdot V(t)
$$


where $\omega(t)$ is the output frequency of the DFB laser, $\omega_{0}$ is the initial start sweep optical frequency, $K_{L}(i)$ is the LDC current dependent gain, $K_{0}$ is the LDC external modulation voltage to current coefficient, and $V(t)$ is the output modulation voltage waveform from the AWG. To simplify the equation, a sweep coefficient $B(i)$ can be defined as:

$$
B(i)=K_{L}(i) \cdot K_{0}
$$

An extra MZI interferometer with two 3-dB couplers and a delay of 28.4 ns was constructed for this iteration process. The laser sweep speed $v(t)$, related with laser frequency $\omega(t)$, can then be measured using this MZI with a delay length of $\tau_{d}$ :

$$
v(t)=\frac{d \omega(t)}{d t}=B(i) \frac{d V(t)}{d t}=\frac{\omega_{B P D}(t)}{\tau_{d}},
$$

where $\omega_{B P D}(t)$ is the radio frequency output of the BPD after the MZI.

The iteration process is as follows: For the $\mathrm{n}^{\text {th }}$ round of sweep $(n \geq 0)$ using the $\mathrm{n}^{\text {th }}$ AWG modulation waveform $V_{n}(t)$, the $\mathrm{n}^{\text {th }}$ laser sweep speed $v_{n}(t)$ can be measured based on eq. (3). Using the $\mathrm{n}^{\text {th }}$ sweep coefficient $B_{n}(i)$, the next round $(\mathrm{n}+1)^{\text {th }}$ sweep coefficient $B_{n+1}(i)$ can be generated:

$$
B_{n+1}(i)=\frac{v_{n}(t)}{\bar{v}} B_{n}(i),(n \geq 0)
$$

where $\bar{v}$ is the target sweep speed and initial sweep coefficient $B_{0}(i)=\bar{v}$. The $(\mathrm{n}+1)^{\text {th }}$ AWG modulation voltage waveform $V_{n+1}(t)$ can then be determined based on eq.(3):

$$
V_{n+1}(t)=\int \frac{\bar{v}}{B_{n+1}(i)} d t,(n \geq 0)
$$

For the first sweeping round ( $\mathrm{n}=0)$, the initial AWG output waveform $V_{0}(t)$ is set as a ramp function from 0 to 1 Volt over $1 \mathrm{~ms}$. The resulting laser optical sweep speed $v_{0}(t)$ is measured using a short-time FFT algorithm and plotted in Figure 6.2 (a). The target 

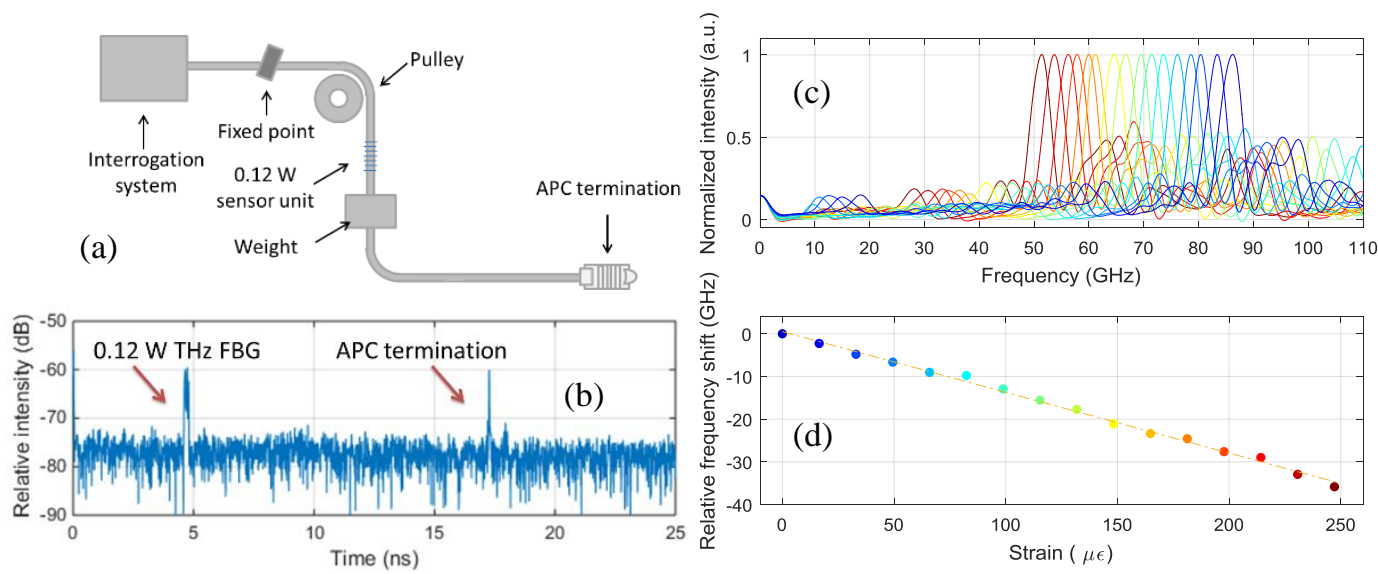

Figure 6.3 Strain tests using the chirp-DFB laser source: (a) strain sensing test setup; (b) time domain reflection signals of the DUT with a $0.12 \mathrm{~W} \mathrm{THz} \mathrm{FBG;} \mathrm{(c)} \mathrm{frequency} \mathrm{domain} \mathrm{interferograms} \mathrm{with}$ varying applied strain values; (d) resulting frequency shift at the varying applied strain values.

frequency sweep speed was set to $140 \mathrm{GHz} / \mathrm{ms}$. After 6 rounds of feedback predistortion iterations, the resulting measured laser sweep speed is plotted in Figure 6.2 (b). The DFB laser sweep from $0.1 \mathrm{~ms}$ to $1 \mathrm{~ms}$ is used to interrogate the THz FBG sensors, where the sweep speed is between 130 and $150 \mathrm{GHz} / \mathrm{ms}$. After re-sampling the data using the reference clock arm, $6 \mathrm{k}$ data points are generated, covering an interrogation bandwidth of $110 \mathrm{GHz}$. A $1 \mathrm{~ms}$ DC voltage output follows the $1 \mathrm{~ms}$ precalibrated waveform to allow the laser sweep speed to return to zero.

A Ti:sapphire femtosecond laser micromachining system (Coherent, Inc.) was employed to fabricate the THz FBG sensing units $[24,73]$. The pitch length was set to $1 \mathrm{~mm}$, resulting in a $100 \mathrm{GHz}$ resonation period, which can be fully covered by the

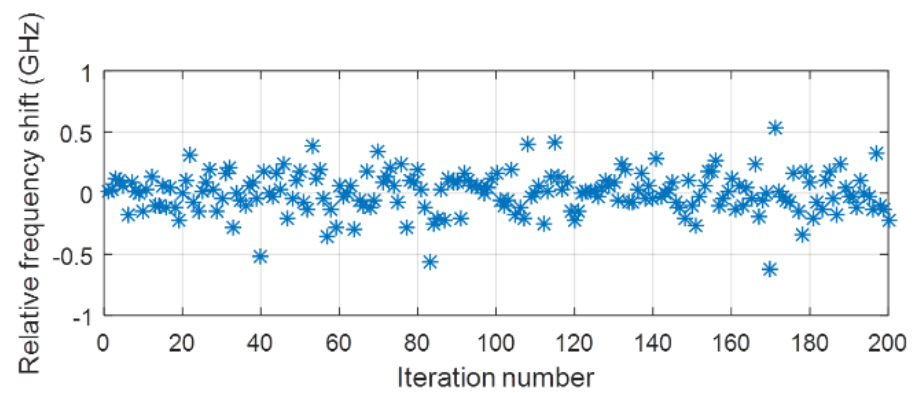

Figure 6.4 Stability test results using 200 iterative measurements 
interrogator. Multiple THz FBGs can be spliced in-line to perform distributed sensing measurements. A C-OFDR system is then used to gather frequency-domain data from the THz FBGs. By employing different band-pass filters, the reflected frequency domain data from each multiplexed THz FBG can be isolated. Using self-mixing and a low pass filter, the frequency domain interferograms $S$ of a THz FBG can be extracted and expressed as:

$$
S=2 \eta^{2} r^{2} I_{r e f}{ }^{2} \sum_{i=0}^{N-1} \sum_{j=0}^{N-1} \cos [2 \beta(i-j) \Delta z]
$$

where $\eta$ is the light-to-voltage conversion coefficient of the photodiode, $r$ is the reflection coefficient of the $\mathrm{THz}$ FBG reflectors, $I_{\text {ref }}$ is the light intensity of the reference arm, $\beta$ is the propagation constant, $N$ is the number of reflectors within the $\mathrm{THz}$ FBG, and $\Delta z$ is the pitch length. The weak reflectivity feature of THz FBG sensors results in very low insertion loss and ignorable cross-talk among THz FBG sensors in a large array so that substantially more sensing elements can be cascaded in-line along one fiber probe using time-domain multiplexing method. The reflected spectrums of demultiplexed THz FBG sensors can be overlapped. The central frequency of a particular THz FBG can be calculated using this equation. Strain applied to the fiber under test expands or contracts the $\mathrm{THz}$ FBG reflector elements, resulting in a phase shift of the $\mathrm{THz}$ FBG interferogram. These phase shifts are ultimately used as a method of distributed strain quantification [74].

\section{Experimental Results and Discussions}

In order to experimentally investigate the chirp-DFB laser-based interrogator system, a strain test was conducted. One THz FBG with a pitch length of $1 \mathrm{~mm}$ and 20 

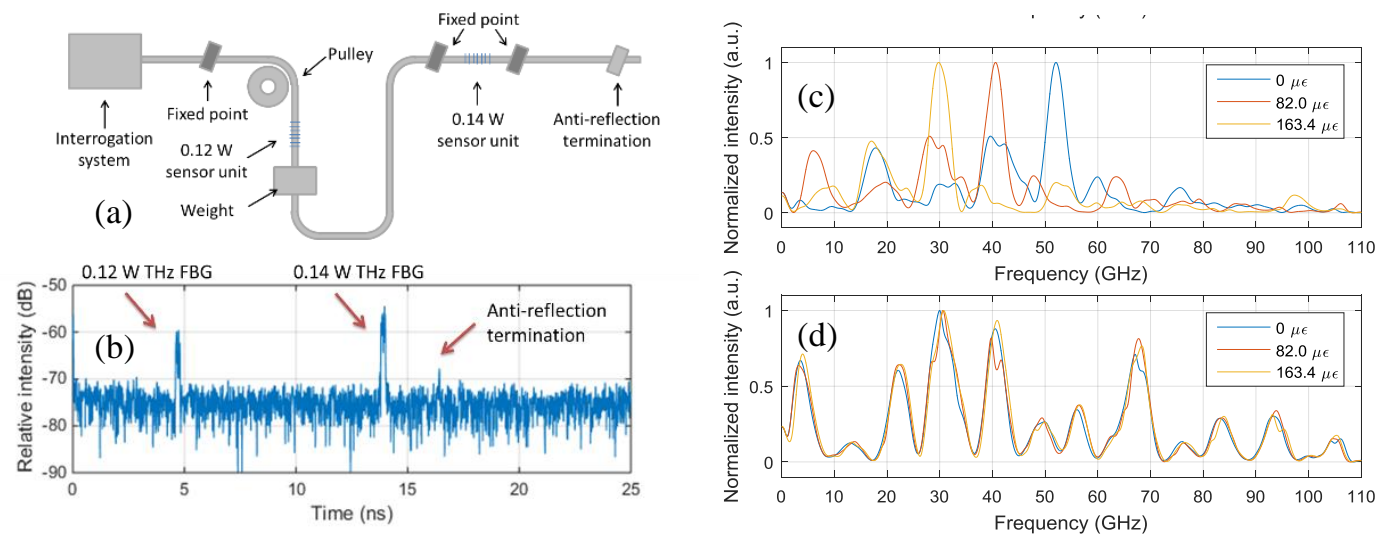

Figure 6.5 Multiplexing test using two THz FBG sensors: (a) multiplexing test setup; (b) time domain reflection signals of the DUT with both the $0.12 \mathrm{~W}$ THz FBG and the $0.14 \mathrm{~W} \mathrm{THz} \mathrm{FBG;} \mathrm{(c)} \mathrm{frequency}$ shift interferograms of the $0.12 \mathrm{~W}$ sensor with different strain values applied; (d) frequency interferograms of the $0.14 \mathrm{~W}$ sensor with no additional strain applied during the same period.

reflector points was fabricated using $0.12 \mathrm{~W}$ femtosecond laser power and connected to the interrogation system. One end of the sensor under test was secured to an optical bench while the other end was left free to hang, shown in Figure 6.3 (a). Weights were sequentially added to the free end of the fiber at $1.4 \mathrm{~g}$ intervals; in total, $21 \mathrm{~g}$ of weights $(247.2 \mu \varepsilon)$ were added to the free end of the sensor under test [75]. The DFB laser was modulated with the pre-calibrated AWG waveform shown in Figure 6.2 (b), covering a total bandwidth of $110.0 \mathrm{GHz}$. The time domain reflected signal, calculated using a Fourier transform algorithm, is plotted in Figure 6.3 (b). The relative intensity was calibrated using an optical fiber APC connector as a $-60 \mathrm{~dB}$ reference. The resulting frequency domain shifted interferograms are plotted in Figure 6.3 (c), and strain test results are plotted in Figure 6.3 (d). A linear relationship was found between the accumulated strain and frequency shift, with an $\mathrm{R}^{2}$ value of 0.996 and strain sensitivity of $-0.142 \mathrm{GHz} / \mu \varepsilon$, which is similar to the sensors fabricated using unmodified silicabased optical fibers [76]. A stability test was then conducted in which the sensor under test was fixed to an optical bench with no strain changes applied. 200 groups of 
measurements were gathered; the frequency domain interferogram shifts were determined, shown in Figure 4, and resulted in a standard deviation of $1.167 \mu \varepsilon$.

To demonstrate multiplexing capability, another $1 \mathrm{~mm}$ pitch length, 20-point $\mathrm{THz}$ FBG was fabricated using $0.14 \mathrm{~W}$ femtosecond laser power and cascaded in-line with the first $\mathrm{THz}$ FBG, as shown in the schematic setup in Figure 5 (a) and the time-domain plot in Figure 6.5 (b). The $0.14 \mathrm{~W}$ sensor has a higher reflectivity compared with the $0.12 \mathrm{~W}$ sensor due to the higher femtosecond laser fabrication power. The $0.12 \mathrm{~W}$ sensor was left free to hang and strains of $82 \mu \varepsilon$ and $163.4 \mu \varepsilon$ applied in a similar manner to the previous strain test, while the $0.14 \mathrm{~W}$ sensor was fixed on the optical bench with no additional strain applied over the course of the test. The resulting frequency domain interferograms of the $0.12 \mathrm{~W}$ sensor and the $0.14 \mathrm{~W}$ sensor are shown in Figure 6.5 (c) and (d). With strain applied, a clear frequency domain interferogram shift trend was observed with the $0.12 \mathrm{~W}$ sensor; during that time, no shift was observed in the interferograms of the $0.14 \mathrm{~W}$ sensor to which no additional strain was applied, indicating good multiplexing capability. Of note, ideally the frequency domain interferogram of periodic grating structures should contain only one strong peak within each resonation period. This was not true of the $0.14 \mathrm{~W} \mathrm{THz} \mathrm{FBG;} \mathrm{the} \mathrm{authors} \mathrm{surmise}$ that this lack of a clear peak was the result of imperfections introduced during fabrication. The precise mechanism of this lack of a clear peak for this particular 0.14 W THz FBG will be the subject of future research; however, this lack did not prevent the authors from observing frequency shifts during testing, as evidenced in Figure 6.5 (d). 
Although the chirp-DFB lasers are a suitable method of interrogating THz FBGs, they present several limitations. The maximum interrogation range in the frequency domain is limited by the sweep bandwidth of the chirp-DFB laser, which is approximately $100 \mathrm{GHz}$; consequently, large strain changes with frequency shifts greater than $100 \mathrm{GHz}$ cannot be resolved. Additionally, the precise starting optical frequency for each frequency sweep may vary; a calibration method that precisely tunes the starting frequency will be investigated in subsequent work. Although the intensity output of laser does vary due to the current injection modulation, the fact that changing output intesnity did not prevent the interrogation to measure multiplexed strain changes using Thz FBGs. Optical or electrical automatic gain control module can be added to potentially solve this problem for particular applications.

\section{Conclusions}

To conclude, this letter reports a chirped DFB laser-based interrogator for $\mathrm{THz}$ FBGs. A DFB laser with current injection modulation was employed as the frequency sweep source used to interrogate two THz FBGs. A feedback iteration method and an auxiliary clock were applied to increase the sweep linearity. $110 \mathrm{GHz}$ interrogation bandwidth within $1 \mathrm{~ms}$ was achieved. Strain testing and multiplexing testing were conducted to experimentally investigate the system. The results of these tests

demonstrate that this system holds substantial potential as a method of dynamic distributed strain sensing, particularly for applications in which increased sampling speed is of particular benefit. 
7. Digitally controlled chirped pulse laser for sub-terahertz-range fiber structure interrogation

\author{
by \\ Zhen Chen, Gerald Hefferman and Tao Wei
}

published in

Optics Letters, vol. 42, no. 5, pp. 1007-1010, Mar. 2017 


\begin{abstract}
This letter reports a sweep velocity-locked laser pulse generator (SV-LLPG) controlled using a digital phase locked loop (DPLL) circuit. This design is used for the interrogation of sub-terahertz-range fiber structures for sensing applications that require real-time data collection with mm-level spatial resolution. A distributed feedback laser (DFB) was employed to generate chirped laser pulses via injection current modulation. A DPLL circuit was developed to lock the optical frequency sweep velocity. A high-quality linearly chirped laser pulse with a frequency excursion of $117.69 \mathrm{GHz}$ at optical communication band was demonstrated. The system was further adopted to interrogate a continuously distributed sub-THz-range fiber structure (Sub-THz-FS) for sensing applications. A strain test was conducted in which the Sub-THz-FS showed a linear response to longitudinal strain change with predicted sensitivity. Additionally, temperature testing was conducted in which a heat source was used to generate a temperature distribution along the fiber structure to demonstrate its distributed sensing capability. A Gaussian temperature profile was measured using the described system and tracked in real-time as the heat source was moved.
\end{abstract}

\title{
Introduction
}

Sub-terahertz-range fiber structures (sub-THz-FS) have demonstrated their feasibility for distributed strain and temperature sensing applications [24, 44, 64, 71]. By definition, a sub-THz-FS is an optical fiber-inline structure with characteristic geometries in the millimeter or sub-millimeter range that can be interrogated using sub-THz bandwidths in the optical frequency band[74]. Uniquely, sub-THz-FSs allow systems to simultaneously achieve distributed strain and temperature measurements with high-accuracy and high 
spatial resolution using a narrow interrogation bandwidth. Previously, the interrogation system of sub-THz-FSs was based on coherent optical frequency domain reflectometry (COFDR) technique where the key component was a highly coherent swept laser source, specifically, an external cavity tunable laser (ECL). An ECL tunes its lasing wavelength via modulating the physical length of an externally-coupled cavity. Recent progress in micro-electro-mechanical systems (MEMS) technologies has led to miniaturized ECLs that can be as compact as conventional semiconductors [77]. The unique advantage of ECLs include high coherence length and mode-hop-free broadband tuning (>100 nm or $>12.5$ $\mathrm{THz}$ at the $1.55 \mu \mathrm{m}$ band). Their disadvantages include inconsistent sweep velocity, nonrepeatable starting wavelength, high system complexity with geometrically coupled moving optical components, and high cost. Several additional components are used to compensate for these limitations: an auxiliary sampling clock (k-clock) is employed to accommodate nonlinear sweep speeds and a wavelength reference gas cell is used to calibrate starting wavelength $[66,78]$. Although effective, these methods add complexity, cost, and increased device footprint to ECLs.

In contrast, a distributed feedback laser (DBF) is also capable of mode-hop-free wavelength tuning via modulating its injection current, without the need for moving geometric-optic components. However, there are two critical fundamental challenges associated with using this frequency sweep technique for C-OFDR-based applications: (1) a limited tuning bandwidth ( 100 GHz), and (2) a nonlinear relationship between injection current and laser frequency, leading to inconsistent sweep velocities. The challenge of limited bandwidth, which restricts the spatial resolution of many C-OFDR applications, is readily overcome using sub-THz-FSs due to their unique, proven ability to facilitate narrow 
interrogation bandwidth operation $[24,44,74]$. Thus, inconsistent sweep velocity represents the key remaining challenge precluding the use of tunable DFB lasers for subTHz-FS sensor interrogation. Efforts have been made to overcome this remaining limitation by implementing an auxiliary sampling clock; however, due to the Nyquist criterion, the delay line for the interferometer used in the sampling clock must be at least four times longer than the total length of sensing arm. This long delay line makes the interrogation system more susceptible to ambient noise, and, given the same sweep velocity, necessitates the use of high frequency electronics, resulting in increased design complexity and system cost.

This letter described an alternative approach that actively linearizes the frequency sweep in order to overcome the remaining challenge of inconsistent sweep velocity directly, allowing for purely-electronically modulated DFB lasers to be used for sub-THzFS sensor interrogation. A sweep velocity-locked laser pulse generator (SV-LLPG) based on a digital phase locked loop (DPLL) design was theoretically developed and prototyped. Using this approach, a tuning bandwidth of $117.69 \mathrm{GHz}$ was achieved over $8.3 \mathrm{~ms}$. A highly consistent sweep velocity of $14.2 \mathrm{GHz} / \mathrm{ms}$ was maintained within each chirped pulse. The standard deviation of the starting frequency was measured to be $109 \mathrm{MHz}$, corresponding to a strain sensing instability of $0.75 \mu \varepsilon$, or a temperature sensing instability of $0.08{ }^{\circ} \mathrm{C}$, obviating the necessity of starting frequency calibration of any kind for most applications. The SV-LLPG prototype was used to interrogate a sub-THz-FS. A linear strain response was obtained with a sensitivity of $-0.1436 \mathrm{GHz} / \mu \varepsilon$, which agrees with previously reported results obtained using an ECL. Additionally, a soldering iron was 
employed as a heat source to form a temperature distribution along a continuously cascaded sub-THz-FS array to demonstrate its high spatial resolution distributed sensing capability.

\section{Operation Mechanism}

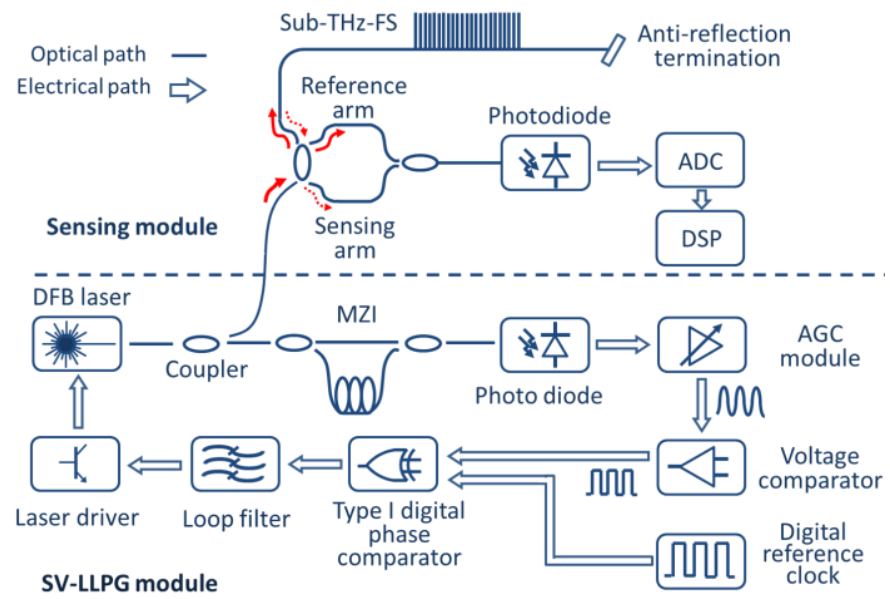

Figure 7.1 Schematic of the proposed Sub-THz-FS interrogation system using SV-LLPG. (ADC: analog-to-digital converter, DSP: digital signal processing unit, MZI: Mach-Zehnder Interferometer, AGC: automatic gain control amplifier.)

A schematic of the described interrogation system is shown in Figure 7.1. A DFB laser serves as the laser source, which is modulated using a time-varying voltage signal through a laser driver circuit. An isolator is placed at the output of the laser to eliminate reflection. Using a 90/10 coupler (CPL), $10 \%$ of the power is directed into a fixed MZI to monitor sweep speed, while $90 \%$ of the remaining power is sent into the sensing module (upper part of Figure 7.1) to activate and interrogate the sub-THz-FSs. The MZI has a fixed delay, $\tau_{\mathrm{d}}$, of $10.58 \mathrm{~ns}$. Under the assumption that the DFB laser is operated with an ideal constant sweep velocity, the AC-coupled current output of the photo diode after the MZI as a function of time, $\mathrm{i}(\mathrm{t})$, can be expressed as:

$$
i(t)=\frac{A(t)^{2}}{8} \eta \cos \left[2 \pi\left(f_{0}+v t\right) \tau_{d}\right]
$$

where $\mathrm{A}(\mathrm{t})$ is the amplitude of the electric field directed into the MZI as a function of time, $\eta$ is the light-to-voltage conversion coefficient of the photodiode, $f_{0}$ is the starting 

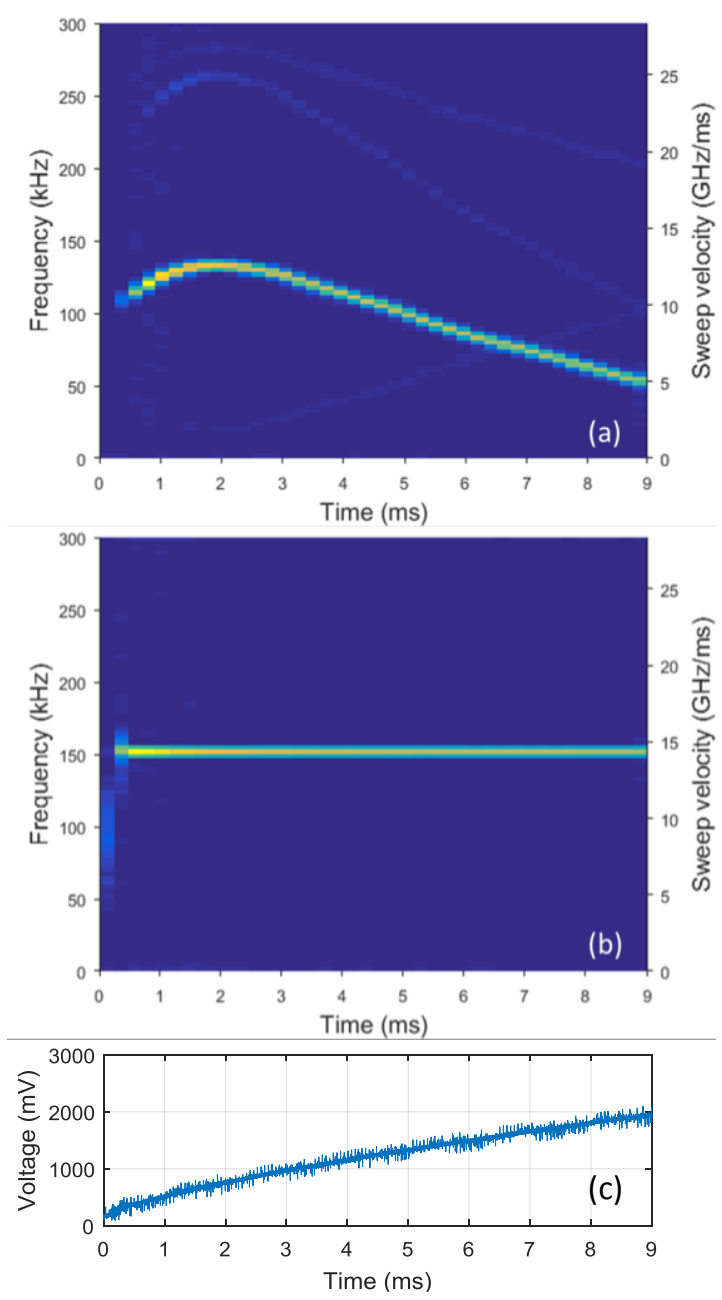

Figure 7.2 Spectrogram of AGC output within a chirped laser pulse: (a) free running; (b) sweep velocity locked; (c) modulation waveforms when the laser sweep velocity is locked.

frequency of the DFB laser sweep, $v$ is the optical frequency sweep velocity, and $t$ is time. Light passing through the MZI generates a beat frequency in the radio frequency (RF) range, which is linearly proportional to the optical frequency sweep velocity given a fixed MZI delay, $\tau_{\mathrm{d}}$. Due to the current injection modulation, the intensity of the DFB laser output varies as a function of time. To account for this effect, an automatic gain control (AGC) transimpedance amplifier is used to adjust the amplitude of the AC-coupled signal output of the photodiode. A voltage comparator is used to convert the analog beat signals generated by the MZI into digital signals, which are then compared to a high-quality digital reference clock with a frequency, $f_{R}$, of $150 \mathrm{kHz}$. Phase errors are then extracted via a type 
I digital phase comparator. A loop filter is used to convert the digital phase error signal into a laser control signal, which is then fed into the laser driver to complete the control loop. It is worth noting that the SV-LLPG can be considered a digital version of an optical phase locked loop (OPLL), and obviates the need for optical amplitude feedback control of analog OPLLs $[72,79]$. Compared with analog mixers, digital phase comparators are inexpensive and less sensitive to noise. Additionally, no polarization maintaining devices were included in the entire system.

A spectrogram of the AGC output during a chirped laser pulse under free-running, open loop operation (when the AGC output is disconnected from the voltage comparator) is shown in Figure 7.2 (a). After closing the control loop, the output of AGC - and thus laser sweep velocity - is locked during each chirped laser pulse. When locked, the AGC is in phase with the digital reference clock; the locked optical frequency sweep velocity, $v$, can therefore be expressed as:

$$
v=\frac{f_{R}}{\tau_{d}}
$$

Given the fixed MZI delay and reference frequency, the locked sweep velocity is calculated to be $\sim 14.2 \mathrm{GHz} / \mathrm{ms}$. Figure 7.2 (b) shows the AGC output within a chirped pulse under the sweep velocity locked condition. The total locking period within a chirped pulse is $\sim 8.3 \mathrm{~ms}$, leading to an optical interrogation bandwidth of $117.69 \mathrm{GHz}$. Figure 7.3 (a) shows the Fourier transform of the AGC output over the span of $8.3 \mathrm{~ms}$ under locked condition; over that span, a signal-to-noise ratio (SNR) above $50 \mathrm{~dB}$ was achieved. During testing, a resting period of $5 \mathrm{~ms}$ followed each $9 \mathrm{~ms}$ sweep in order to discharge the capacitors in the loop filter, resulting in a total $14 \mathrm{~ms}$ for each complete pulse cycle and a reputation rate of $71 \mathrm{~Hz}$. To determine the noise of the system, 1 second of data with 71 
chirped laser pulses was recorded. The Fourier transform of this data is plotted in Figure 7.3 (b). A $71 \mathrm{~Hz}$ frequency period was observed due to the reputation rate. The full width at half maximum (FWHM) of the peak envelope using a Gaussian curve fit was measured to be $116 \mathrm{~Hz}$.

A homodyne configuration was constructed using two $2 \times 23-\mathrm{dB}$ couplers, depicted in the sensing module of Figure 7.1. The input light is split into two paths via the first coupler, with one path serving as the reference arm and the other path directed into the sensing arm including a sub-THz-FS array. The sensing arm was terminated using an anti-reflection cut. The reflected light from sub-THz-FSs was then combined with light from the reference arm through the second coupler. A photodetector and a single channel AC-coupled 12-bit ADC was used to record the resulting data. The sampling rate of the ADC was set to 8 $\mathrm{MSa} / \mathrm{s}$ with a matched anti-aliasing filter. The digitized raw data was then fed into a DSP module.
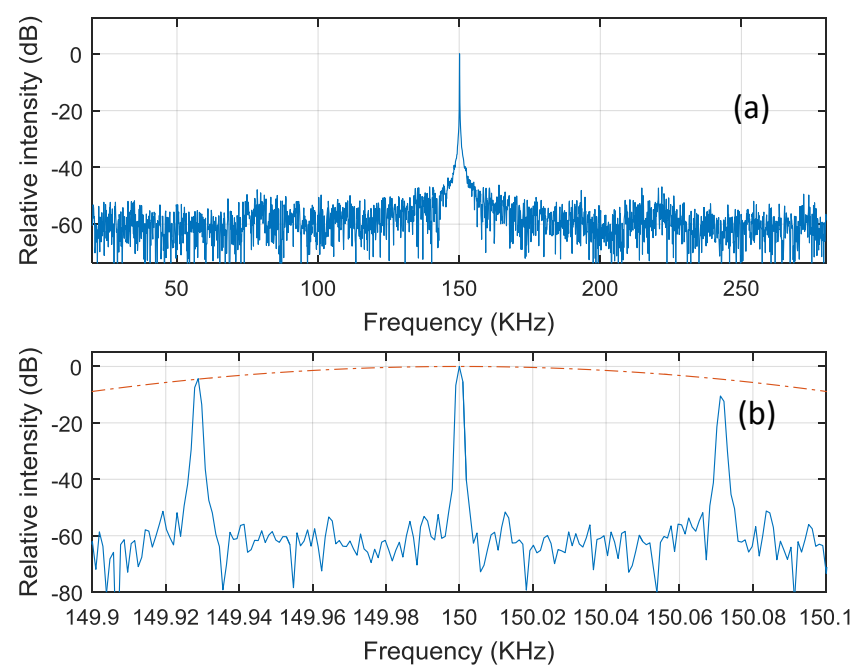

Figure 7.3 Quality of sweep velocity locking: (a) Fourier transform of the AGC output over the locked span of $8.3 \mathrm{~ms}$; (b) a Gaussian curve fit applied to the measure the FWHM of the Fourier transform of a chirped pulse train over 1 second.

\section{Experimental Results and Discussions}


In order to investigate the sensing capability of the described concept, a 20-pt periodic weak reflection sub-THz-FS array with a $1 \mathrm{~mm}$ pitch length was fabricated along a single mode fiber (SMF-28, Corning, Inc.) using a Ti: Sapphire femtosecond laser (Coherent, Inc.) $[26,38]$. During interrogation and signal processing, the sub-THz-FS array was considered as 9 cascaded sub-THz-grating sensor units using a 4-mm wide moving Butterworth bandpass filter with a step size of 2-mm. In this case, each sensor unit contains 4 reflection peaks. This signal processing method has been systematically investigated in the previous publications $[24,44,74]$. The interferograms of the target sensor units were extracted using a self-mixing method and a low-pass filter. Changes in strain or
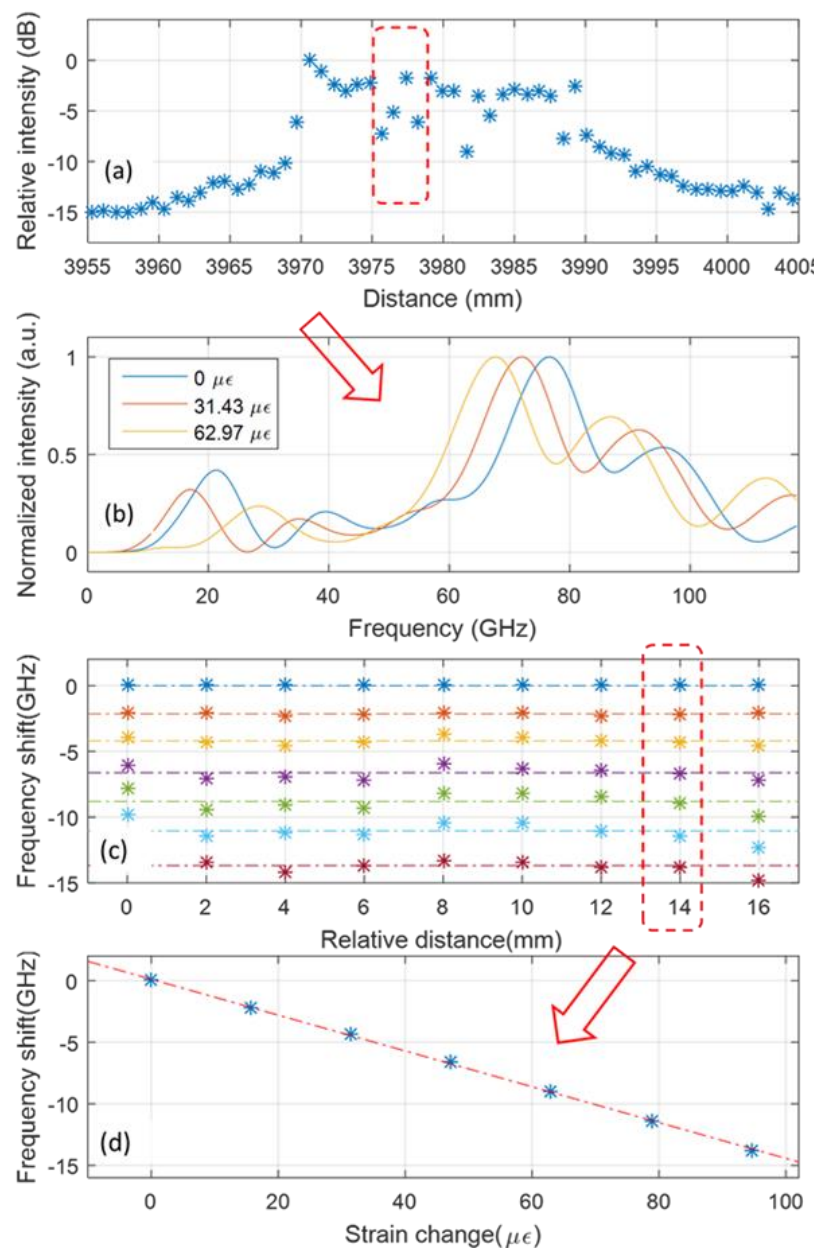

Figure 7.4 Static strain test: (a) time domain reflections of DUT; (b) interferograms of the sensor unit between $3975 \mathrm{~mm}$ and $3979 \mathrm{~mm}$ with varied strain applied; (c) strain test results for all 9 sensor units; (d) strain test results for 8th sensor unit 
temperature along the optical fiber result in optical path length (OPL) changes between the weak reflectors of the sub-THz-FS array. Thus, a phase-shift in the resulting interferogram can be used to measure strain and temperature along the sensor probe.

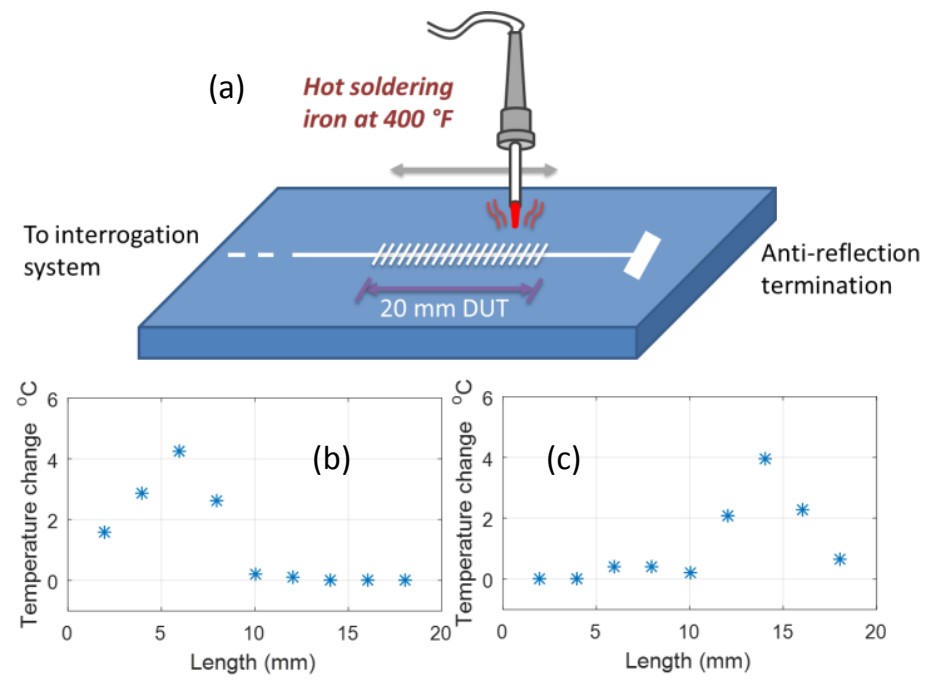

Figure 7.5 Distributed temperature test: (a) schematic; (b), (c) testing results [Visualization 1]

To demonstrate the strain sensing capability of the system, a series of static strain tests were conducted. One end of the device under test (DUT) was secured to an optical bench while the other end was left free to hang. Weights were sequentially added to the free end of the fiber at $1.33 \mathrm{~g}$ intervals; in total $7.98 \mathrm{~g}$ of weights were added to the free end of the DUT, resulting in a strain change of $93.92 \mu \varepsilon$. The SV-LLPG system was set using the parameters described above, resulting in a total sweeping bandwidth of $117.69 \mathrm{GHz}$. The resulting distance domain signals, calculated using a Fourier transform and in which the sensor structures can be identified between $3969 \mathrm{~mm}$ and $3989 \mathrm{~mm}$, are plotted in Figure 7.4 (a). The individual reflection peaks of the sub-THz-FS array elements cannot be resolved due to the narrow bandwidth used for interrogation. The measured frequency domain interferograms of the $3^{\text {rd }}$ senor unit between $3975 \mathrm{~mm}$ and $3979 \mathrm{~mm}$ are plotted in Figure 7.4 (b). The strain test results for all 9 sensor units are plotted in Figure 7.4 (c), and 
the results of the $8^{\text {th }}$ sensor unit specifically are plotted in Figure 7.4 (d). Linear results were observed for all sensor units, with the least linear having a R2 value of 0.9950 . The mean strain sensitivity across all sensing elements was calculated to be $-0.1436 \mathrm{GHz} / \mu \varepsilon$ with a standard deviation of $0.0078 \mathrm{GHz} / \mu \varepsilon$. A stability test was conducted in which the sub-THz-FS array was affixed to an optical bench and no strain changes applied. 100 groups of measurements were collected; the maximum standard deviation among these sensor units was calculated to be $0.16 \mathrm{GHz}$, corresponding to a detection limit of $1.11 \mu \varepsilon$. The starting sweep frequency was evaluated by measuring the starting frequency of the entire system over 1000 captures, and the standard deviation of start frequency was 109 MHz.

In order to demonstrate the distributed sensing capability of the system, a dynamic temperature test was conducted. A schematic illustration of the testing setup is shown in Figure 7.5 (a). The interrogator setup was identical to that of the static strain test. A heat source (a soldering iron tip heated to $400^{\circ} \mathrm{F}$ ) was then placed approximately $2 \mathrm{~mm}$ from the sub-THz-FS array. The heat source was free to move along the direction of the array in order to introduce a changing temperature distribution at various locations along the optical fiber. A graphical unit interface (GUI) was employed to generate temperature profile along the Sub-THz-FS array in real-time at a refresh rate of $2.5 \mathrm{~Hz}$. Figure 7.5 (b) and (c) show the measured temperature distributions with the heat source at different locations. A video file documenting this distributed temperate testing is included as a supplement to this manuscript.

\section{Conclusions}


To conclude, this manuscript reports a SV-LLPG enabled by a DPLL circuit to interrogate a sub-THz-FS sensor array for distributed sensing with millimeter-level spatial resolutions. A highly linearly chirped pulse over a bandwidth of $117.69 \mathrm{GHz}$ at optical frequency was achieved within $8.3 \mathrm{~ms}$ at a repetition rate of $71 \mathrm{~Hz}$. The locked sweep velocity was $14.2 \mathrm{GHz} / \mathrm{ms}$. The proposed apparatus was built around a purely electronically-modulated DFB laser source. A static strain test was conducted and linear results observed, with a mean sensitivity of $-0.1436 \mathrm{GHz} / \mu \varepsilon$ and a detection limit of 1.11 $\mu \varepsilon$. In order to evaluate the distributed sensing capability of the system, a dynamic temperature sensing test was conducted, which demonstrated the system's ability to measure changing temperature distributions in real-time. This system holds substantial potential as a means of achieving rapid, real-time measurement of distributed temperature and strain data for a broad range of sensing applications. 
8. Field-programmable gate array-controlled sweep velocity-locked laser pulse generator

by

Zhen Chen, Gerald Hefferman and Tao Wei

published in

Optical Engineering, SPIE, vol. 56, no. 5, pp. 054102, May. 2016. 


\section{Abstract}

This manuscript reports a FPGA-controlled sweep velocity-locked laser pulse generator (SV-LLPG) design based on an all-digital phase-locked loop (ADPLL). A distributed feedback (DFB) laser with modulated injection current was used as a swept-frequency laser source. An open loop pre-distortion modulation waveform was calibrated using a feedback iteration method to initially improve frequency sweep linearity. An ADPLL control system was then implemented using a field programmed gate array (FPGA) to lock the output of a Mach-Zehnder interferometer that was directly proportional to laser sweep velocity to an on-board system clock. Using this system, linearly chirped laser pulses with a sweep bandwidth of $111.16 \mathrm{GHz}$ were demonstrated. Further testing evaluating the sensing utility of the system was conducted. In this test, the SV-LLPG served as the swept laser source of an optical frequency domain reflectometry (OFDR) system was used to interrogate a sub-terahertz range fiber structure (sub-THz-FS) array. A static strain test was then conducted and linear sensor results were observed.

\section{Introduction}

Frequency modulated continuous wave (FMCW) reflectometry or optical frequency domain reflectometry (OFDR) $[2,5,54]$, is a well-established frequency domain measurement method for optical component characterization and optical fiber distributed sensing. This technique allows distance domain information to be obtained from frequency domain intensity data via a Fourier transform. A key component of this FMCW reflectometry system is a swept-frequency laser source. A variety of laser sources have been investigated for this purpose, including temperature and piezoelectrically tuned Nd: YAG ring lasers [67, 68], external cavity lasers (ECLs) [24, 25, 
44, 80, 81], piezo-electrically tuned fiber grating lasers [70], and chirped distributed feedback (DFB) lasers [69].

Among these sources, the chirped DFB laser represents a particularly promising candidate for narrow-bandwidth interrogation applications. The output frequency of DFB lasers can be controlled using injection current modulation without the need for any moving mechanical components, resulting in a fast repetition rate over a sweeping bandwidth of $\sim 100 \mathrm{GHz}$. Other beneficial features of chirped DFB lasers include single longitudinal mode output, good laser coherence length $(\sim \mathrm{km})$, and low cost. However, there are several drawbacks limiting chirped DFB lasers as elements of FMCW/OFDR systems. Chief among these is a non-linear relationship between input current and output frequency, leading to non-linear optical sweep speeds. To compensate this nonlinearity, a pre-distortion waveform can be generated based on a feedback iteration method [82]. However, this method only fractionally enhances the linearity of the resulting optical sweep speed. An auxiliary clock or 'k-clock' with a fixed optical delay can also be applied to resample the data and correct for non-linear sweep speeds [82]. However, this sampling clock method increases both system sampling and signal processing complexity.

A closed loop control system based on an optical phase-locked loop (OPLL) that modifies laser output frequency in real-time offers an alternative approach to precisely controlling optical sweep speed $[72,83]$. Recently, a digital-controlled chirped pulse laser based on a digital phase-locked loop (DPLL) design was reported based on modular electronic design [84]. A digital phase comparator (XOR gate) was utilized to extract phase errors between the output of a Mach-Zehnder interferometer, which 
converted laser sweep speed to a radio-frequency signal, and a reference oscillator. The system generated a highly linear frequency sweep, and its utility as a source for high spatial resolution fiber sensing applications was demonstrated. However, while this system successfully demonstrated the concept, there remain engineering challenges, stemming from the fact that analog systems are susceptible to noise and DC drifts in comparison to similar digital systems $[85,86]$. More importantly, modular design results in relatively high power consumption, large size and weight of the final product.

This manuscript reports an alternative design for a sweep velocity-locked laser pulse generator (SV-LLPG) using an all-digital phase-lock loop (ADPLL), which has the potential to surmount several of the previous engineering challenges facing modular DPLL design. The ADPLL is constructed such that all components, including the phase comparator, loop controller, and reference frequency synthesizer, are digitally generated using logic gates and integrated in an IC chip. This design was then implemented using a field-programmable gate array (FPGA) in which all digital components were synchronized using the same on-chip clock to minimize phase noise. The FPGA chip used in this design is from Xilinx Zyqn 7000 series, and the DAC is a Texas Instruments DAC121S101 with 12-bit resolution and a sampling rate of $1 \mathrm{MSa} / \mathrm{s}$. Using this approach, a sweeping bandwidth of $111.16 \mathrm{GHz}$ over $9 \mathrm{~ms}$ has been demonstrated. A highly consistent sweep velocity of $12.35 \mathrm{GHz} / \mathrm{ms}$ is maintained within each chirped pulse. The standard deviation of the starting frequency was measured to be $106.7 \mathrm{MHz}$, corresponding to a strain sensing instability of $0.79 \mu \varepsilon$. In order to evaluate the potential of the ADPLL-based SV-LLPG as an element of an OFDR system, the system was used to interrogate a sub-THz-FS array. Highly linear results and a sensitivity of -0.1346 
$\mathrm{GHz} / \mu \varepsilon$ were observed, which agrees well with previously reported sensing results obtained using an ECL [24, 44].

\section{Operation Mechanism}

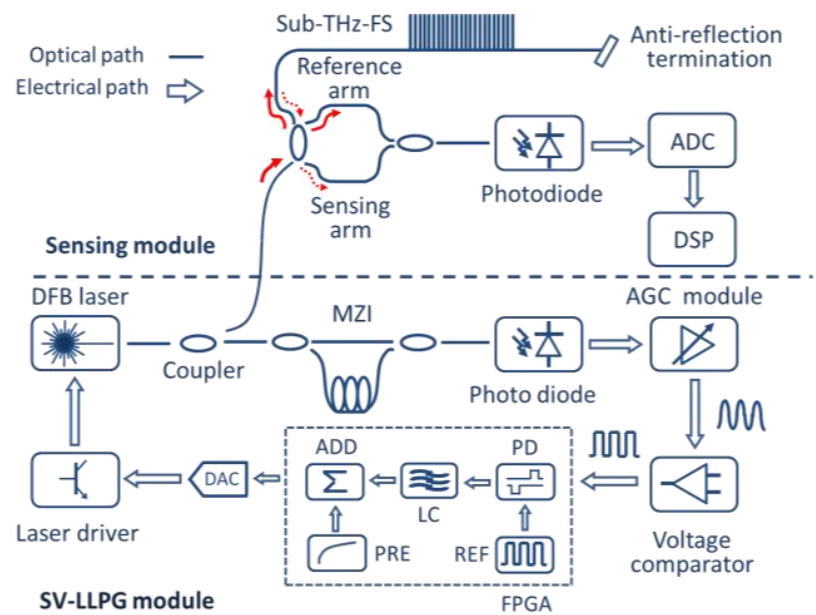

Figure 8.1 Schematic of proposed all-digital OPLL system with sensing modulate and control module (MZI: Mach-Zehnder interferometer; FPGA: field-programmable logic array; DAC: digital-to-analog converter; ADD: adder; PRE: pre-distortion curve; LC: loop controller; REF: reference frequency clock; PD: type-II phase detector; ADC: analog-to-digital converter; DSP: digital signal processing unit).

A schematic of the described interrogation system is shown in Figure 8.1. In the SV-LLPG module, a DFB laser is employed as the frequency sweep source, which is injection current-modulated using a time-varying voltage via a laser control circuit. An isolator is placed at the laser output to eliminate reflection. Using a 90/10 coupler, 10\% of the output power is directed in to the MZI and $90 \%$ of the power into the sensing module to interrogate the sub-THz-FS array. The MZI is constructed with two 3-dB couplers with a constant delay, $\tau_{d}$, of $11.334 \mathrm{~ns}$. Under the assumption that the DFB laser is operated at a constant sweep velocity, the AC-coupled current output $\mathrm{i}(\mathrm{t})$ at the photo diode after the MZI as a function of time can be expressed as:

$$
i(t)=\frac{A(t)^{2}}{8} \eta \cos \left[2 \pi\left(f_{0}+v t\right) \tau_{d}\right]
$$


where $\mathrm{A}(\mathrm{t})$ is the electrical-field amplitude directed into the MZI as a function of time, $\eta$ is the responsivity of the photo diode, $f_{-} 0$ is the initial frequency of the DFB laser during sweeping, $v$ is the optical sweep velocity, and $t$ is the time. A beat frequency in the radio frequency $(\mathrm{RF})$ range less than $250 \mathrm{kHz}$, which is linearly proportional to laser sweep velocity, is generated through this fixed delay MZI. Due to the current injection modulation, the intensity of the DFB laser output varies as a function of time. To account for this effect, an automatic gain control (AGC) transimpedance amplifier is used to adjust the amplitude of AC-coupled photodiode output signal. This photodiode has a bandwidth of $1 \mathrm{MHz}$. A high-speed voltage comparator with a bandwidth of 50 $\mathrm{MHz}$ is used to convert the analog beat signals into digital signals, which is sent into a digital input port of a FPGA evaluation board with a $100 \mathrm{MHz}$ system clock. In order to improve the initial laser sweep linearity before phase locking, a pre-distortion voltage waveform is pre-calibrated using a feedback iteration method [82], resulting in an output frequency sweep velocity at about $12.5 \mathrm{GHz} / \mathrm{ms}$. This open loop pre-distortion voltage waveform is then stored within the FPGA memory. A type-II phase comparator, constructed with two D flip-flops and an AND gate [87], is used to extract the phase difference between the input digital signal and the on-board reference frequency clock $f_{R}$ at $140 \mathrm{kHz}$. The resulting phase error signals are then fed into a loop controller constructed using an integrator to further modify the pre-distortion current modulation waveform. The digital output of the FGPA is converted to an analog signal using a digital-to-analog converter (DAC) module with a refresh cycle of $1 \mu$ s and sent to the laser driver circuit. 

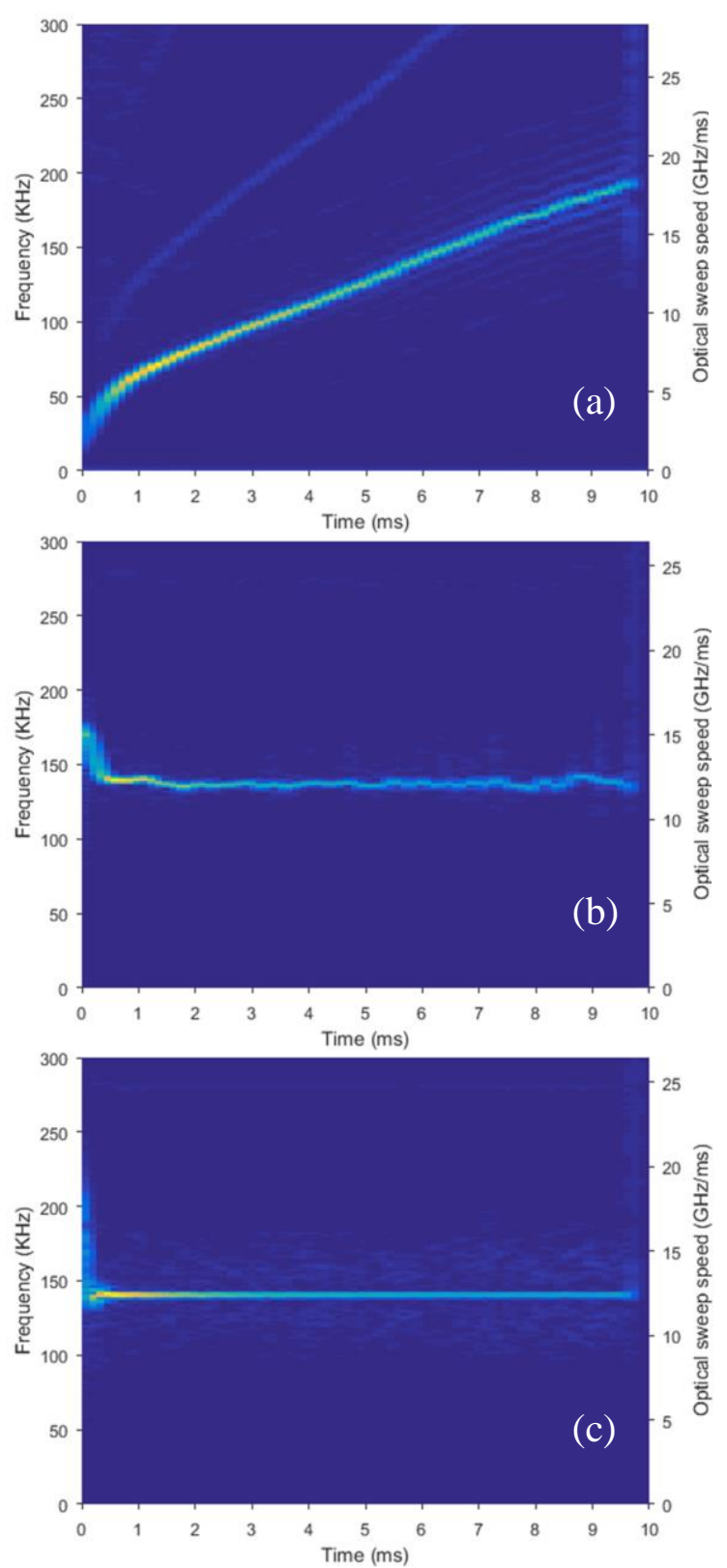

Figure 8.2 Measured frequency spectrum of AGC output within a chirped laser pulse: (a) free running with ramp input; (b) free running with calibrated pre-distortion input; (c) sweep velocity locked.

Spectrograms of the AGC output during a chirped laser pulse under the free-running open loop case (when the phase errors are not fed into the loop controller) with an unmodified ramp input and under the pre-calibrated modulation waveform case are shown in Figure 8.2 (a) and (b). Sweep linearity is substantially improved using the predistortion curve compared with the initial ramp waveform. After closing the control 
loop (i.e. when the phase errors are fed into the loop controller), the laser sweep velocity is locked during each chirped laser pulse. When locked, the AGC output signal is in phase with the digital reference clock and the locked optical frequency sweep velocity $v$ can be expressed as:

$$
v=\frac{f_{R}}{\tau_{d}}
$$

Given the fixed MZI delay length $\tau_{-} d$ and the digital reference clock frequency $f_{R}$, the locked sweep velocity is calculated to be $12.35 \mathrm{GHz} / \mathrm{ms}$. Figure 8.2 (c) shows the AGC output within a chirped pulsed under the locked condition. The total locking period within the chirped pulse is approximately $9 \mathrm{~ms}$, leading to an optical sweeping bandwidth of $111.16 \mathrm{GHz}$. Figure 8.3 (a) shows the Fourier transform of the AGC output over the span of 9 ms under the locked condition; over that span, a signal-tonoise ratio (SNR) above $35 \mathrm{~dB}$ was achieved. During testing, a resting period of $10 \mathrm{~ms}$ followed each $10 \mathrm{~ms}$ sweep in order discharge the capacitor within the laser driver circuit, resulting in a total period of $20 \mathrm{~ms}$ for each complete pulse cycle and a reputation rate of $50 \mathrm{~Hz}$. The output of the chirped laser pulse generator for 5 complete cycles at the photodetector is plotted in Figure 8.3 (b). To determine the noise of the system, 1 second of data with 50 chirped laser pulses was recorded. The Fourier transform of this data is plotted in Figure 8.3 (c). A center frequency of $139.667 \mathrm{kHz}$ was found. A 50 $\mathrm{Hz}$ frequency period was observed due to the repetition rate described above. The full width at half maximum (FWHM) of the peak envelope using a Gaussian curve fit was measured to be $90 \mathrm{~Hz}$. 

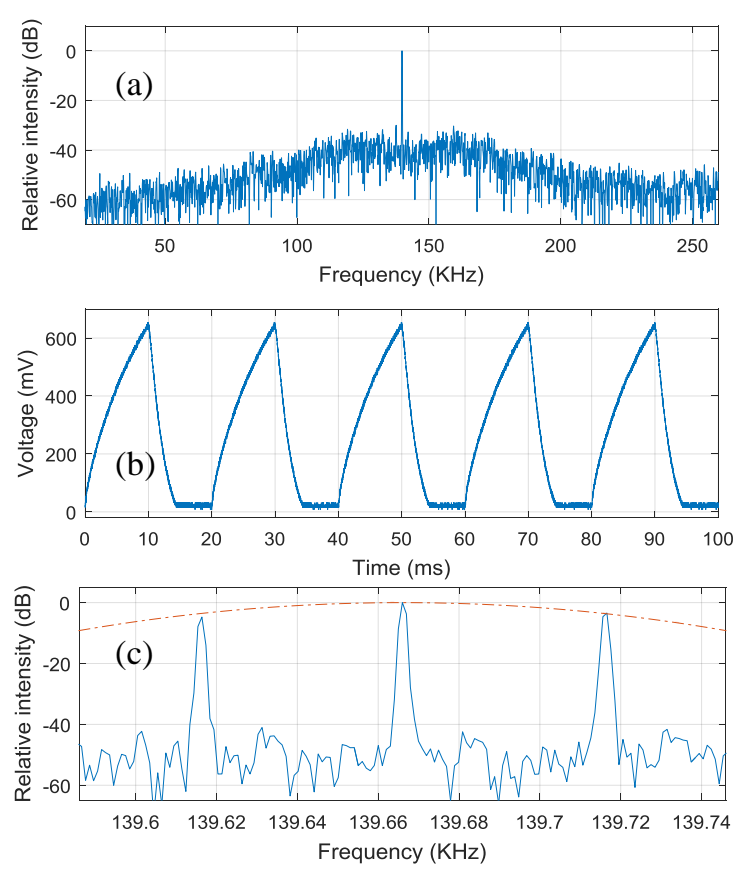

Figure 8.3 (a) Fourier transform of the AGC output over the locked span of 9 ms; (b) output of the laser pulse generator with 5 complete cycles; (c) a Gaussian curve fit applied to measure the FWHM of the Fourier transform of a chirped pulse train over 1 second.

Along the sensing module, a homodyne interferometry structure is constructed using two $2 \times 23-\mathrm{dB}$ couplers as shown in Figure 8.1. The input light is split into two paths via the first coupler, with one serving as reference arm and the other path directed into the sensing arm, which includes a sub-THz-FS array. The sensing arm is terminated using an anti-reflection cut. The reflected light from the sub-THz-FS is then combined with light from reference arm via the second coupler. A photodetector and a single channel AC-coupled 8-bit analog-to-digital converter (ADC) is used to record the resulting data. The sampling rate of the $\mathrm{ADC}$ is set to $8 \mathrm{MSa} / \mathrm{s}$ with a match anti-aliasing filter. The digitized raw data is then fed into a DSP module.

\section{Experimental Results and Discussions}



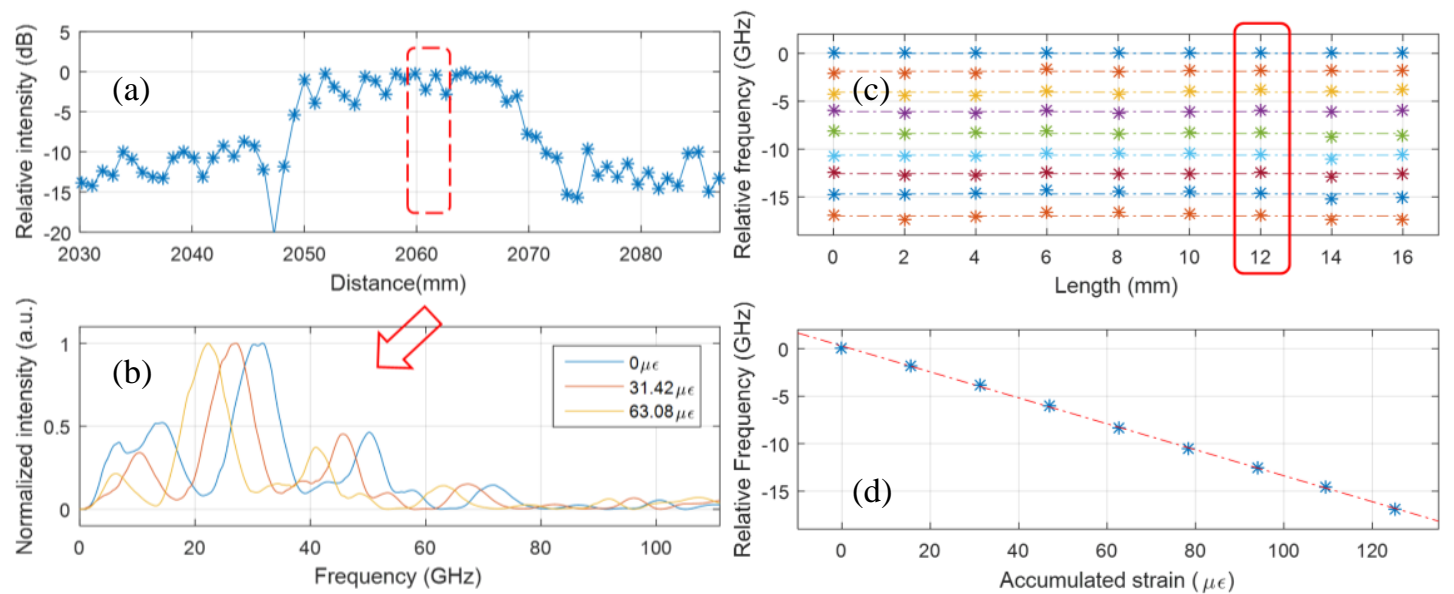

Figure 8.4 Static strain test: (a) time domain reflections of DUT; (b) interferograms of the sensor unit between $1779 \mathrm{~mm}$ and $1783 \mathrm{~mm}$ with varied strain applied; (c) strain test results for all 9 sensor units; (d) strain test results of for 7 th sensor unit.

In order to investigate the sensing capability of the described SV-LLPG system, a 20-pt periodic weak reflection sub-THz-FS array with a $1 \mathrm{~mm}$ pitch length was fabricated along a single mode fiber (SMF-28, Corning, Inc.) using a Ti: Sapphire femtosecond laser micromachining system (Coherent, Inc.) [24, 44, 64, 73]. During interrogation and signal processing, this sub-THz-FS array was considered to be 9 cascaded sub-THz- grating sensor units using a 4-mm wide moving Butterworth bandpass filter with a step size of 2-mm. Each sensor unit contains 4 reflection peaks. This signal processing method has been systematically investigated in the previous publications $(7,22)$. A self-mixing method and a low pass filter is applied to extract the resulting interferograms. Changes in strain along the optical fiber result in optical path length (OPL) changes between the weak reflectors, which generate a phase-shift in the interferograms that are used to measure strain changes along the sensor probe.

To evaluate the strain sensing capability of the system, a series of static strain tests were conducted. One end of the fiber under test (FUT) was secured to an optical bench while the other end was left free to hang. Weights were sequentially added to the free 
end of the fiber at $1.33 \mathrm{~g}$ intervals; in total $10.64 \mathrm{~g}$ of weights were added to the free end of the FUT, resulting in a strain change of $125.23 \mu \varepsilon$. The SV-LLPG system was set using the parameters described above, resulting in a total sweeping bandwidth of 111.16 GHz. The resulting distance domain signals, calculated using a Fourier transform and in which the sensor structures can be identified between $2047 \mathrm{~mm}$ and $2067 \mathrm{~mm}$, are plotted in Figure 8.4 (a). Due to the limited interrogation bandwidth, the individual reflection peaks of the sub-THz-FS array elements cannot be resolved. The measured frequency domain interferograms of the 7th sensor unit between $2059 \mathrm{~mm}$ and $2063 \mathrm{~mm}$ are plotted in Figure 8.4 (b). The strain test results for all 9 sensor units are plotted in Figure 8.4 (c), and the results of the 7th sensor unit specifically are plotted in Figure 8.4 (d). Linear results were observed for all sensor units, with the least linear having a $\mathrm{R}^{2}$ value of 0.9986 . The mean strain sensitivity across all sensing elements was calculated to be $-0.1346 \mathrm{GHz} / \mu \varepsilon$ with a standard deviation of $0.0026 \mu \varepsilon$. The start sweep frequency was evaluated by measuring the starting frequency of the entire system over 1000 captures, and the standard deviation of start frequency was $106.7 \mathrm{MHz}$.

\section{Conclusions}

To conclude, this manuscript reports a FPGA-controlled sweep velocity-locked laser pulse generator (SV-LLPG) design. A DFB laser is employed as the sweep source and an ADPLL control system is used to lock the laser sweep velocity to an on-board reference clock. Highly linear chirped laser pulses with a bandwidth of $111.16 \mathrm{GHz}$ were demonstrated. A sweep velocity of $12.35 \mathrm{GHz} / \mathrm{ms}$ was achieved for $9 \mathrm{~ms}$ within each chirped pulse at a $50 \mathrm{~Hz}$ pulse repetition rate. To investigate system sensing utility, the SV-LLPG prototype was used as an element of an OFDR system to interrogate a 
sub-THz-FS array. A static strain test was conducted and highly linear results were observed.

The proposed device holds the promise to deliver a low size, weight and power (SWaP) and affordable interrogator for distributed fiber sensing applications. In addition, the FPGA based design makes it easier to be integrated and adopted for various applications in the future. 


\section{List of References}

[1] J. Huang, L. Hua, X. Lan, T. Wei, and H. Xiao, "Microwave assisted reconstruction of optical interferograms for distributed fiber optic sensing," Optics Express, vol. 21, pp. 18152-18159, 2013/07/29 2013.

[2] X. Bao and L. Chen, "Recent progress in distributed fiber optic sensors," Sensors, vol. 12, pp. 8601-8639, 2012.

[3] G. M. Prisco, "Fiber optic shape sensing," ed: Google Patents, 2012.

[4] L. Thévenaz, S. Foaleng Mafang, K.-Y. Song, S. H. Chin, J.-C. Beugnot, N. Primerov, and M. Tur, "Recent progress towards centimetric spatial resolution in distributed fibre sensing," in Proc. SPIE, 2010, p. 765309.

[5] M. Froggatt and J. Moore, "High-spatial-resolution distributed strain measurement in optical fiber with Rayleigh scatter," Applied Optics, vol. 37, pp. 1735-1740, 1998.

[6] W. Wang, J. Gong, B. Dong, D. Y. Wang, T. J. Shillig, and A. Wang, "A large serial time-division multiplexed fiber Bragg grating sensor network," Lightwave Technology, Journal of, vol. 30, pp. 2751-2756, 2012.

[7] K. Hotate and K. Kajiwara, "Proposal and experimental verification of Bragg wavelength distribution measurement within a long-length FBG by synthesis of optical coherence function," Optics Express, vol. 16, pp. 7881-7887, 2008/05/26 2008.

[8] D. Johnson, "Novel Optical Fibers-Draw-tower process creates high-quality FBG arrays," Laser Focus World, vol. 48, p. 53, 2012. 
[9] F. Shen, W. Peng, K. L. Cooper, G. Pickrell, and A. Wang, "UV-induced intrinsic Fabry-Perot interferometric fiber sensors," 2004, pp. 47-56.

[10] L. Yuan, J. Huang, X. Lan, H. Wang, L. Jiang, and H. Xiao, "All-in-fiber optofluidic sensor fabricated by femtosecond laser assisted chemical etching," Optics letters, vol. 39, pp. 2358-2361, 2014/04/15 2014.

[11] Y. Zhang, L. Yuan, X. Lan, A. Kaur, J. Huang, and H. Xiao, "High-temperature fiber-optic Fabry\&\#x2013;Perot interferometric pressure sensor fabricated by femtosecond laser," Optics letters, vol. 38, pp. 4609-4612, 2013/11/15 2013.

[12] W. Wang, D. Ding, N. Chen, F. Pang, and T. Wang, "Quasi-Distributed IFPI Sensing System Demultiplexed With FFT-Based Wavelength Tracking Method," IEEE Sensors Journal, vol. 12, pp. 2875-2880, 2012.

[13] J. Huang, X. Lan, M. Luo, and H. Xiao, "Spatially continuous distributed fiber optic sensing using optical carrier based microwave interferometry," Optics Express, vol. 22, pp. 18757-18769, 2014/07/28 2014.

[14] K. O. Hill and G. Meltz, "Fiber Bragg grating technology fundamentals and overview," Journal of lightwave technology, vol. 15, pp. 1263-1276, 1997.

[15] T. Wei, S. Wu, J. Huang, H. Xiao, and J. Fan, "Coaxial cable Bragg grating," Applied Physics Letters, vol. 99, p. 113517, 2011.

[16] S. Wu, T. Wei, J. Huang, H. Xiao, and J. Fan, "Modeling of Coaxial Cable Bragg Grating by Coupled Mode Theory," IEEE Transactions on Microwave Theory and Techniques, vol. 62, pp. 2251-2259, 2014.

[17] J. Huang, T. Wei, X. Lan, J. Fan, and H. Xiao, "Coaxial cable Bragg grating sensors for large strain measurement with high accuracy," in SPIE Smart 
Structures and Materials+ Nondestructive Evaluation and Health Monitoring, 2012, pp. 83452Z-83452Z-9.

[18] A. D. Kersey, M. A. Davis, H. J. Patrick, M. LeBlanc, K. Koo, C. Askins, M. Putnam, and E. J. Friebele, "Fiber grating sensors," Journal of lightwave technology, vol. 15, pp. 1442-1463, 1997.

[19] P. H. Siegel, "Terahertz technology in biology and medicine," in Microwave Symposium Digest, 2004 IEEE MTT-S International, 2004, pp. 1575-1578.

[20] S. F. Zhou, L. Reekie, H. P. Chan, Y. T. Chow, P. S. Chung, and K. Man Luk, "Characterization and modeling of Bragg gratings written in polymer fiber for use as filters in the THz region," Optics Express, vol. 20, pp. 9564-9571, 2012.

[21] G. Yan, A. Markov, Y. Chinifooroshan, S. M. Tripathi, W. J. Bock, and M. Skorobogatiy, "Resonant $\mathrm{THz}$ sensor for paper quality monitoring using $\mathrm{THz}$ fiber Bragg gratings," Optics letters, vol. 38, pp. 2200-2202, 2013.

[22] J. Huang, X. Lan, T. Wei, Q. Han, Z. Gao, Z. Zhou, and H. Xiao, "Radio frequency interrogated actively mode-locked fiber ring laser for sensing application," Optics letters, vol. 37, pp. 494-496, 2012/02/15 2012.

[23] T. Wei, J. Huang, X. Lan, Q. Han, and H. Xiao, "Optical fiber sensor based on a radio frequency Mach-Zehnder interferometer," Optics letters, vol. 37, pp. 647-649, 2012/02/15 2012.

[24] Z. Chen, L. Yuan, G. Hefferman, and T. Wei, "Ultraweak intrinsic Fabry-Perot cavity array for distributed sensing," Optics letters, vol. 40, pp. 320-323, 2015. 
[25] Z. Chen, Y. Zeng, G. Hefferman, Y. Sun, and T. Wei, "FiberID: Molecular-level secret for identification of things," in IEEE Workshop on Information Forensics and Security (WIFS'14), Altalanta GA, Dec 3-5 2014, 2014.

[26] L. Yuan, T. Wei, Q. Han, H. Wang, J. Huang, L. Jiang, and H. Xiao, "Fiber inline Michelson interferometer fabricated by a femtosecond laser," Optics letters, vol. 37, pp. 4489-4491, 2012/11/01 2012.

[27] Y. Wang, J. Gong, D. Y. Wang, B. Dong, W. Bi, and A. Wang, "A QuasiDistributed Sensing Network With Time-Division-Multiplexed Fiber Bragg Gratings," IEEE Photonics Technology Letters, vol. 23, pp. 70-72, 2011.

[28] R. R. Gattass, "Femtosecond-laser interactions with transparent materials: applications in micromachining and supercontinuum generation," Harvard University Cambridge, Massachusetts, 2006.

[29] C. B. Schaffer, A. Brodeur, J. F. García, and E. Mazur, "Micromachining bulk glass by use of femtosecond laser pulses with nanojoule energy," Optics letters, vol. 26, pp. 93-95, 2001.

[30] D. Du, X. Liu, G. Korn, J. Squier, and G. Mourou, "Laser-induced breakdown by impact ionization in $\mathrm{SiO} 2$ with pulse widths from $7 \mathrm{~ns}$ to $150 \mathrm{fs}$," Applied Physics Letters, vol. 64, pp. 3071-3073, 1994.

[31] Y. Kondo, K. Nouchi, T. Mitsuyu, M. Watanabe, P. G. Kazansky, and K. Hirao, "Fabrication of long-period fiber gratings by focused irradiation of infrared femtosecond laser pulses," Optics letters, vol. 24, pp. 646-648, 1999. 
[32] T. Wei, Y. Han, H.-L. Tsai, and H. Xiao, "Miniaturized fiber inline Fabry-Perot interferometer fabricated with a femtosecond laser," Optics letters, vol. 33, pp. 536-538, 2008.

[33] A. Martinez, M. Dubov, I. Khrushchev, and I. Bennion, "Direct writing of fibre Bragg gratings by femtosecond laser," Electronics Letters, vol. 40, pp. 11701172, 2004.

[34] A. Kaur, S. E. Watkins, J. Huang, L. Yuan, and H. Xiao, "Microcavity strain sensor for high temperature applications," Optical Engineering, vol. 53, pp. 017105-017105, 2014.

[35] Y. Zhang, J. Huang, X. Lan, L. Yuan, and H. Xiao, "Simultaneous measurement of temperature and pressure with cascaded extrinsic Fabry-Perot interferometer and intrinsic Fabry-Perot interferometer sensors," Optical Engineering, vol. 53, p. 067101, 2014.

[36] Y. Zhang, L. Yuan, X. Lan, A. Kaur, J. Huang, and H. Xiao, "High-temperature fiber-optic Fabry-Perot interferometric pressure sensor fabricated by femtosecond laser," Optics letters, vol. 38, pp. 4609-4612, 2013.

[37] L. Yuan, X. W. Lan, J. Huang, and H. Xiao, "Femtosecond Laser Processing of Glass Materials for Assembly-Free Fabrication of Photonic Microsensors," in Advances in Science and Technology, 2014, pp. 166-173.

[38] Y. Lei, L. Xinwei, H. Jie, W. Hanzheng, J. Lan, and X. Hai, "Comparison of Silica and Sapphire Fiber SERS Probes Fabricated by a Femtosecond Laser," Photonics Technology Letters, IEEE, vol. 26, pp. 1299-1302, 2014. 
[39] J. Huang, X. Lan, H. Wang, L. Yuan, T. Wei, Z. Gao, and H. Xiao, "Polymer optical fiber for large strain measurement based on multimode interference," Optics letters, vol. 37, pp. 4308-4310, 2012.

[40] J. Huang, X. Lan, A. Kaur, H. Wang, L. Yuan, and H. Xiao, "Reflection-based phase-shifted long period fiber grating for simultaneous measurement of temperature and refractive index," Optical Engineering, vol. 52, pp. 014404014404, 2013.

[41] J. Huang, X. Lan, A. Kaur, H. Wang, L. Yuan, and H. Xiao, "Temperature compensated refractometer based on a cascaded SMS/LPFG fiber structure," Sensors and Actuators B: Chemical, vol. 198, pp. 384-387, 2014.

[42] J. Huang, L. Hua, X. Lan, and H. Xiao, "Fiber optic distributed sensing technology based on microwave reconstructed optical interferograms," in Frontiers in Optics, 2013, p. FW6B. 1.

[43] J. Huang, X. Lan, Y. Song, Y. Li, L. Hua, and H. Xiao, "Microwave interrogated sapphire fiber Michelson interferometer for high temperature sensing," Photonics Technology Letters, IEEE, vol. PP, pp. 1-1, 2015.

[44] Z. Chen, L. Yuan, G. Hefferman, and T. Wei, "Terahertz Fiber Bragg Grating for Distributed Sensing," Photonics Technology Letters, IEEE, vol. 27, pp. 1084-1087, 2015.

[45] Z. Shu Fan, L. Reekie, C. Yuk Tak, C. Hau Ping, and L. Kwai Man, "PhaseShifted Fiber Bragg Gratings for Terahertz Range," Photonics Technology Letters, IEEE, vol. 24, pp. 1875-1877, 2012. 
[46] Z. Qi, L. Nan, T. Fink, L. Hong, P. Wei, and H. Ming, "Fiber-Optic Pressure Sensor Based on <formula formulatype="inline"> <img src="/images/tex/525.gif" alt="|pi"> </formula>-Phase-Shifted Fiber Bragg Grating on Side-Hole Fiber," Photonics Technology Letters, IEEE, vol. 24, pp. 1519-1522, 2012.

[47] C. Zhen, Z. Yongbo, G. Hefferman, and S. Yan, "FiberID: molecular-level secret for identification of things," in Information Forensics and Security (WIFS), 2014 IEEE International Workshop on, 2014, pp. 84-88.

[48] A. Othonos, "Fiber Bragg gratings," Review of Scientific Instruments, vol. 68, pp. 4309-4341, 1997.

[49] G. A. Miller, C. G. Askins, and G. A. Cranch, "Interferometric interrogation of a multicore fiber, two-axis inclinometer," in 20th International Conference on Optical Fibre Sensors, 2009, pp. 75032R-75032R-4.

[50] C. G. Askins, G. A. Miller, and E. J. Friebele, "Bend and twist sensing in a multicore optical fiber," in IEEE Lasers and Electro-Optics Society, 2008. LEOS 2008. 21st Annual Meeting of the, 2008, pp. 109-110.

[51] G. A. Miller, "Fabrication of a Multifiber Optical Inclinometer," Photonics Technology Letters, IEEE, vol. 27, pp. 1289-1292, 2015.

[52] K. K. C. Lee, A. Mariampillai, M. Haque, B. A. Standish, V. X. D. Yang, and P. R. Herman, "Temperature-compensated fiber-optic 3D shape sensor based on femtosecond laser direct-written Bragg grating waveguides," Optics Express, vol. 21, pp. 24076-24086, 2013/10/07 2013. 
[53] C. Waltermann, A. Doering, M. Köhring, M. Angelmahr, and W. Schade, "Cladding waveguide gratings in standard single-mode fiber for 3D shape sensing," Optics letters, vol. 40, pp. 3109-3112, 2015/07/01 2015.

[54] W. Eickhoff and R. Ulrich, "Optical frequency domain reflectometry in singlemode fiber," Applied Physics Letters, vol. 39, pp. 693-695, 1981.

[55] B. Soller, D. Gifford, M. Wolfe, and M. Froggatt, "High resolution optical frequency domain reflectometry for characterization of components and assemblies," Optics Express, vol. 13, pp. 666-674, 2005.

[56] M. E. Froggatt, D. K. Gifford, S. T. Kreger, M. S. Wolfe, and B. J. Soller, "Distributed Strain and Temperature Discrimination in Unaltered Polarization Maintaining Fiber," in Optical Fiber Sensors, Cancun, 2006, p. ThC5.

[57] F. Xinyu, Y. Koshikiya, and F. Ito, "Phase-Noise-Compensated Optical Frequency-Domain Reflectometry," Quantum Electronics, IEEE Journal of, vol. 45, pp. 594-602, 2009.

[58] X. Fan, Y. Koshikiya, and F. Ito, "Phase-noise-compensated optical frequency domain reflectometry with measurement range beyond laser coherence length realized using concatenative reference method," Optics letters, vol. 32, pp. 3227$3229,2007 / 11 / 152007$.

[59] D.-P. Zhou, Z. Qin, W. Li, L. Chen, and X. Bao, "Distributed vibration sensing with time-resolved optical frequency-domain reflectometry," Optics Express, vol. 20, pp. 13138-13145, 2012/06/04 2012. 
[60] J. P. Moore and M. D. Rogge, "Shape sensing using multi-core fiber optic cable and parametric curve solutions," Optics Express, vol. 20, pp. 2967-2973, 2012/01/30 2012.

[61] R. G. Duncan, M. E. Froggatt, S. T. Kreger, R. J. Seeley, D. K. Gifford, A. K. Sang, and M. S. Wolfe, "High-accuracy fiber-optic shape sensing," 2007, pp. 65301S-65301S-11.

[62] P. S. Westbrook, K. S. Feder, T. Kremp, T. F. Taunay, E. Monberg, J. Kelliher, R. Ortiz, K. Bradley, K. S. Abedin, D. Au, and G. Puc, "Integrated optical fiber shape sensor modules based on twisted multicore fiber grating arrays," 2014, pp. 89380H-89380H-7.

[63] M. E. Froggatt and R. G. Duncan, "Fiber optic position and/or shape sensing based on rayleigh scatter," ed: Google Patents, 2010.

[64] G. Hefferman, C. Zhen, Y. Lei, and W. Tao, "Phase-Shifted Terahertz Fiber Bragg Grating for Strain Sensing With Large Dynamic Range," Photonics Technology Letters, IEEE, vol. 27, pp. 1649-1652, 2015.

[65] Z. Chen, G. Hefferman, L. Yuan, Y. Song, and T. Wei, "Ultra-weak waveguide modification with intact buffer coating using femtosecond laser pulses," Photonics Technology Letters, IEEE, vol. PP, pp. 1-1, 2015.

[66] B. J. Soller, D. K. Gifford, M. S. Wolfe, and M. E. Froggatt, "High resolution optical frequency domain reflectometry for characterization of components and assemblies," Optics Express, vol. 13, pp. 666-674, 2005/01/24 2005. 
[67] S. Venkatesh, W. V. Sorin, D. K. Donald, and B. L. Heffner, "Coherent FMCW Reflectometry Using A Piezoelectrically Tuned Nd:YAG Ring Laser," in Optical Fiber Sensors, Monterey, California, 1992, p. W34.

[68] W. V. Sorin, D. K. Donald, S. A. Newton, and M. Nazarathy, "Coherent FMCW reflectometry using a temperature tuned Nd:YAG ring laser," IEEE Photonics Technology Letters, vol. 2, pp. 902-904, 1990.

[69] R. Passy, N. Gisin, J. P. v. d. Weid, and H. H. Gilgen, "Experimental and theoretical investigations of coherent OFDR with semiconductor laser sources," Journal of lightwave technology, vol. 12, pp. 1622-1630, 1994.

[70] P. Oberson, B. Huttner, O. Guinnard, L. Guinnard, G. Ribordy, and N. Gisin, "Optical frequency domain reflectometry with a narrow linewidth fiber laser," IEEE Photonics Technology Letters, vol. 12, pp. 867-869, 2000.

[71] Z. Chen, G. Hefferman, L. Yuan, Y. Song, and T. Wei, "Terahertz-range interrogated grating-based two-axis optical fiber inclinometer," Optical Engineering, vol. 55, pp. 026106-026106, 2016.

[72] N. Satyan, A. Vasilyev, G. Rakuljic, V. Leyva, and A. Yariv, "Precise control of broadband frequency chirps using optoelectronic feedback," Optics Express, vol. 17, pp. 15991-15999, 2009/08/31 2009.

[73] Z. Chen, G. Hefferman, L. Yuan, Y. Song, and T. Wei, "Ultraweak Waveguide Modification With Intact Buffer Coating Using Femtosecond Laser Pulses," IEEE Photonics Technology Letters, vol. 27, pp. 1705-1708, 2015. 
[74] Z. Chen, G. Hefferman, and T. Wei, "Terahertz-Range Weak Reflection Fiber Optic Structures for Sensing Applications," IEEE Journal of Selected Topics in Quantum Electronics, vol. PP, pp. 1-1, 2016.

[75] P. Antunes, F. Domingues, M. Granada, and P. André, Mechanical properties of optical fibers: INTECH Open Access Publisher, 2012.

[76] M. Ramakrishnan, G. Rajan, Y. Semenova, and G. Farrell, "Overview of Fiber Optic Sensor Technologies for Strain/Temperature Sensing Applications in Composite Materials," Sensors, vol. 16, p. 99, 2016.

[77] A. Liu and X. Zhang, "A review of MEMS external-cavity tunable lasers," Journal of Micromechanics and Microengineering, vol. 17, p. R1, 2006.

[78] E. D. Moore and R. R. McLeod, "Correction of sampling errors due to laser tuning rate fluctuations in swept-wavelength interferometry," Optics Express, vol. 16, pp. 13139-13149, 2008/08/18 2008.

[79] K. Iiyama, W. Lu-Tang, and H. Ken-Ichi, "Linearizing optical frequency-sweep of a laser diode for FMCW reflectometry," Journal of lightwave technology, vol. 14, pp. 173-178, 1996.

[80] Z. Chen, G. Hefferman, and T. Wei, "Multiplexed displacement fiber sensor using thin core fiber exciter," Review of Scientific Instruments, vol. 86, p. $065004,2015$.

[81] B. Tang, Z. Chen, G. Hefferman, T. Wei, H. He, and Q. Yang, "A Hierarchical Distributed Fog Computing Architecture for Big Data Analysis in Smart Cities," presented at the Proceedings of the ASE BigData \& SocialInformatics 2015, Kaohsiung, Taiwan, 2015. 
[82] Z. Chen, G. Hefferman, and T. Wei, "A Low Bandwidth DFB Laser-Based Interrogator for Terahertz-Range Fiber Bragg Grating Sensors," IEEE Photonics Technology Letters, vol. 29, pp. 365-368, 2017.

[83] P. A. Roos, R. R. Reibel, T. Berg, B. Kaylor, Z. W. Barber, and W. R. Babbitt, "Ultrabroadband optical chirp linearization for precision metrology applications," Optics letters, vol. 34, pp. 3692-3694, 2009/12/01 2009.

[84] Z. Chen, G. Hefferman, and T. Wei, "Digitally controlled chirped pulse laser for sub-terahertz-range fiber structure interrogation," Optics letters, vol. 42, pp. 1007-1010, 2017/03/01 2017.

[85] A. Babu, B. Daya, B. Nagasundaram, and N. Veluchamy, "All digital phase locked loop design and implementation," Project report, University of Florida, Gainesville, FL, vol. 32608, 2009.

[86] V. Kratyuk, P. K. Hanumolu, U. K. Moon, and K. Mayaram, "A Design Procedure for All-Digital Phase-Locked Loops Based on a Charge-Pump PhaseLocked-Loop Analogy," IEEE Transactions on Circuits and Systems II: Express Briefs, vol. 54, pp. 247-251, 2007.

[87] W. Min, W. Zhiping, C. Lei, and Z. Yanlong, "A novel configurable no deadzone digital phase detector design," in APCCAS 2008 - 2008 IEEE Asia Pacific Conference on Circuits and Systems, 2008, pp. 721-724. 